\title{
Modern Theory of Nuclear Forces
}

\author{
E. Epelbaum* \\ Forschungszentrum Jülich, Institut für Kernphysik (IKP-3) and Jülich Center for Hadron Physics, D-52425 Jülich, \\ Germany and \\ Helmholtz-Institut für Strahlen- und Kernphysik (Theorie) and Bethe Center for Theoretical Physics, Universität Bonn, \\ D-53115 Bonn, Germany \\ H.-W. Hammer ${ }^{\dagger}$ \\ Helmholtz-Institut für Strahlen- und Kernphysik (Theorie) and Bethe Center for Theoretical Physics, Universität Bonn, \\ D-53115 Bonn, Germany \\ Ulf-G. Meißner \\ Helmholtz-Institut für Strahlen- und Kernphysik (Theorie) and Bethe Center for Theoretical Physics, Universität Bonn, \\ D-53115 Bonn, Germany and \\ Forschungszentrum Jülich, Institut für Kernphysik (IKP-3) and Jülich Center for Hadron Physics, D-52425 Jülich, \\ Germany
}

\begin{abstract}
Effective field theory allows for a systematic and model-independent derivation of the forces between nucleons in harmony with the symmetries of Quantum Chromodynamics. We review the foundations of this approach and discuss its application for light nuclei at various resolution scales. The extension of this approach to many-body systems is briefly sketched.
\end{abstract}

Commissioned article for Reviews of Modern Physics

\section{Contents}

I. QCD and Nuclear Forces

A. Chiral symmetry

B. Scales in nuclear physics

C. Conventional approaches to the nuclear force problem 4

D. Brief introduction to effective field theory 5

E. First results from lattice QCD

F. Observables and not-so observable quantities

II. EFT for Few-Nucleon Systems: Foundations and Applications

A. EFT with contact interactions and universal aspects

B. Chiral EFT for few nucleons: foundations

C. Chiral EFT for few nucleons: applications

D. The role of the $\Delta$-isobar

E. Few-nucleon reactions involving pions

F. Hyperon-nucleon \& hyperon-hyperon interactions

G. Nuclear lattice simulations

H. Quark mass dependence of nuclear forces and IR limit cycle in QCD

III. Towards a Many-Body EFT for Nuclei

A. In-medium chiral perturbation theory

B. Perturbative chiral nuclear dynamics

C. EFT for halo nuclei

D. $\boldsymbol{V}_{\text {low k }}$ potentials: construction and applications

E. Lattice simulations of many-nucleon systems

IV. Summary and perspectives

Acknowledgments

References

\footnotetext{
*Electronic address: e.epelbaum@fz-juelich.de

${ }^{\dagger}$ Electronic address: hammer@itkp.uni-bonn.de

$\ddagger$ Electronic address: meissner@itkp.uni-bonn.de
}

\section{QCD AND NUCLEAR FORCES}

Within the Standard Model of particle physics, the strong interactions are described by Quantum Chromodynmics (QCD). QCD is a fascinating theory with many intriguing manifestations. Its structure and interactions are governed by a local non-abelian gauge symmetry,

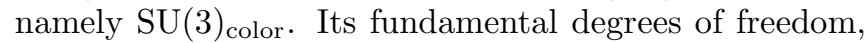
the quarks (the matter fields) and gluons (the force carriers), have never been observed in isolation (confinement). The strong coupling constant $\alpha_{S}$ exhibits a very pronounced running and is of order one in the typical energy scales of nuclear physics. The bound states made from the basic constituents are the hadrons, the strongly interacting particles. The particle spectrum shows certain regularities that can be traced back to the flavor symmetries related to the fermions building up these states. More precisely, there are six quark flavors. These can be grouped into two very different sectors. While the light quarks $(u, d, s)$ are almost massless and thus have to be treated relativistically, bound states made from heavy quarks allow for a precise non-relativistic treatment. In what follows, we will only consider the light quarks at low energies, where perturbation theory in $\alpha_{S}$ is inapplicable (this regime is frequently called "strong QCD"). A further manifestation of strong QCD is the appearance of nuclei, shallow bound states composed of protons, neutrons, pions or strange particles like hyperons. The resulting nuclear forces that are responsible for the nuclear binding are residual color forces, much like the van der Waals forces between neutral molecules. It is the aim of this article to provide the link between QCD and its symmetries, in particular the spontaneously and explicitely 
broken chiral symmetry, and the nuclear forces which will allow to put nuclear physics on firm theoretical grounds and also gives rise to a very accurate calculational scheme for nuclear forces and the properties of nuclei.

This review is organized as follows: In this section, we briefly discuss some of the concepts underlying the chiral effective field theory of the nuclear forces and make contact to ab initio lattice simulations of two-baryon systems as well as to more phenomenological approaches. Sec. II deals with the foundations and applications of nuclear EFT and should be considered the central piece of this review. In particular, tests of these forces in few-nucleon systems are discussed. Attempts to tackle nuclear matter and finite nuclei are considered in sec. III. We end with a short summary and outlook.

\section{A. Chiral symmetry}

First, we must discuss chiral symmetry in the context of QCD. Chromodynamics is a non-abelian $S U(3)_{\text {color }}$ gauge theory with $N_{f}=6$ flavors of quarks, three of them being light $(u, d, s)$ and the other three heavy $(c, b, t)$. Here, light and heavy refers to a typical hadronic scale of about $1 \mathrm{GeV}$. In what follows, we consider light quarks only (the heavy quarks are to be considered as decoupled). The QCD Lagrangian reads

$$
\begin{aligned}
\mathcal{L}_{\mathrm{QCD}} & =-\frac{1}{2 g^{2}} \operatorname{Tr}\left(G_{\mu \nu} G^{\mu \nu}\right)+\bar{q} i \gamma^{\mu} D_{\mu} q-\bar{q} \mathcal{M} q \\
& =\mathcal{L}_{\mathrm{QCD}}^{0}-\bar{q} \mathcal{M} q
\end{aligned}
$$

where we have absorbed the gauge coupling in the definition of the gluon field and color indices are suppressed. The three-component vector $q$ collects the quark fields, $q^{T}(x)=(u(s), d(x), s(x))$. As far as the strong interactions are concerned, the different quarks $u, d, s$ have identical properties, except for their masses. The quark masses are free parameters in QCD - the theory can be formulated for any value of the quark masses. In fact, light quark QCD can be well approximated by a fictitious world of massless quarks, denoted $\mathcal{L}_{\mathrm{QCD}}^{0}$ in Eq. (1.1). Remarkably, this theory contains no adjustable parameter the gauge coupling $g$ merely sets the scale for the renormalization group invariant scale $\Lambda_{\mathrm{QCD}}$. Furthermore, in the massless world left- and right-handed quarks are completely decoupled. The Lagrangian of massless QCD is invariant under separate unitary global transformations of the left- and right-hand quark fields, the so-called chiral rotations, $q_{I} \rightarrow V_{I} q_{I}, V_{I} \in U(3), I=L, R$, leading to $3^{2}=9$ conserved left- and 9 conserved right-handed currents by virtue of Noether's theorem. These can be expressed in terms of vector $(V=L+R)$ and axial-vector $(A=L-R)$ currents

$$
\begin{aligned}
V_{0}^{\mu} & =\bar{q} \gamma^{\mu} q, \quad V_{a}^{\mu}=\bar{q} \gamma^{\mu} \frac{\lambda_{a}}{2} q, \\
A_{0}^{\mu} & =\bar{q} \gamma^{\mu} \gamma_{5} q, \quad A_{a}^{\mu}=\bar{q} \gamma^{\mu} \gamma_{5} \frac{\lambda_{a}}{2} q,
\end{aligned}
$$

Here, $a=1, \ldots 8$, and the $\lambda_{a}$ are Gell-Mann's $S U(3)$ flavor matrices. The singlet axial current is anomalous, and thus not conserved. The actual symmetry group of massless QCD is generated by the charges of the conserved currents, it is $G_{0}=S U(3)_{R} \times S U(3)_{L} \times U(1)_{V}$. The $U(1)_{V}$ subgroup of $G_{0}$ generates conserved baryon number since the isosinglet vector current counts the number of quarks minus antiquarks in a hadron. The remaining group $S U(3)_{R} \times S U(3)_{L}$ is often referred to as chiral $S U(3)$. Note that one also considers the light $u$ and $d$ quarks only (with the strange quark mass fixed at its physical value), in that case, one speaks of chiral $S U(2)$ and must replace the generators in Eq. (1.2) by the Paulimatrices. Let us mention that QCD is also invariant under the discrete symmetries of parity $(P)$, charge conjugation $(C)$ and time reversal $(T)$. Although interesting in itself, we do not consider strong $C P$ violation and the related $\theta$-term in what follows, see e.g. (1).

The chiral symmetry is a symmetry of the Lagrangian of QCD but not of the ground state or the particle spectrum - to describe the strong interactions in nature, it is crucial that chiral symmetry is spontaneously broken. This can be most easily seen from the fact that hadrons do not appear in parity doublets. If chiral symmetry were exact, from any hadron one could generate by virtue of an axial transformation another state of exactly the same quantum numbers except of opposite parity. The spontaneous symmetry breaking leads to the formation of a quark condensate in the vacuum $\langle 0|\bar{q} q| 0\rangle=\left\langle 0\left|\bar{q}_{L} q_{R}+\bar{q}_{R} q_{L}\right| 0\right\rangle$, thus connecting the left- with the right-handed quarks. In the absence of quark masses this expectation value is flavorindependent: $\langle 0|\bar{u} u| 0\rangle=\langle 0|\bar{d} d| 0\rangle=\langle 0|\bar{q} q| 0\rangle$. More precisely, the vacuum is only invariant under the subgroup of vector rotations times the baryon number current, $H_{0}=S U(3)_{V} \times U(1)_{V}$. This is the generally accepted picture that is supported by general arguments (2) as well as lattice simulations of QCD (for a recent study, see (3)). In fact, the vacuum expectation value of the quark condensate is only one of the many possible order parameters characterizing the spontaneous symmetry violation - all operators that share the invariance properties of the vacuum qualify as order parameters. The quark condensate nevertheless enjoys a special role, it can be shown to be related to the density of small eigenvalues of the QCD Dirac operator (see (4) and more recent discussions in $(5 ; 6)), \lim _{\mathcal{M} \rightarrow 0}\langle 0|\bar{q} q| 0\rangle=-\pi \rho(0)$. For free fields, $\rho(\lambda) \sim \lambda^{3}$ near $\lambda=0$. Only if the eigenvalues accumulate near zero, one obtains a non-vanishing condensate. This scenario is indeed supported by lattice simulations and many model studies involving topological objects like instantons or monopoles.

Before discussing the implications of spontaneous symmetry breaking for QCD, we briefly remind the reader of Goldstone's theorem $(7 ; 8)$ : to every generator of a spontaneously broken symmetry corresponds a massless excitation of the vacuum. These states are the Goldstone bosons, collectively denoted as pions $\pi(x)$ in what 
follows. Through the corresponding symmetry current the Goldstone bosons couple directly to the vacuum,

$$
\left\langle 0\left|A^{0}(0)\right| \pi\right\rangle \neq 0 .
$$

In fact, the non-vanishing of this matrix element is a necessary and sufficient condition for spontaneous symmetry breaking. In QCD, we have eight (three) Goldstone bosons for $S U(3)(S U(2))$ with spin zero and negative parity - the latter property is a consequence that these Goldstone bosons are generated by applying the axial charges on the vacuum. The dimensionful scale associated with the matrix element Eq. (1.3) is the pion decay constant (in the chiral limit)

$$
\left\langle 0\left|A_{\mu}^{a}(0)\right| \pi^{b}(p)\right\rangle=i \delta^{a b} F p_{\mu},
$$

which is a fundamental mass scale of low-energy QCD. In the world of massless quarks, the value of $F$ differs from the physical value by terms proportional to the quark masses, to be introduced later, $F_{\pi}=F[1+\mathcal{O}(\mathcal{M})]$. The physical value of $F_{\pi}$ is $92.4 \mathrm{MeV}$, determined from pion decay, $\pi \rightarrow \nu \mu$.

Of course, in QCD the quark masses are not exactly zero. The quark mass term leads to the so-called explicit chiral symmetry breaking. Consequently, the vector and axial-vector currents are no longer conserved (with the exception of the baryon number current)

$$
\partial_{\mu} V_{a}^{\mu}=\frac{1}{2} i \bar{q}\left[\mathcal{M}, \lambda_{a}\right] q, \quad \partial_{\mu} A_{a}^{\mu}=\frac{1}{2} i \bar{q}\left\{\mathcal{M}, \lambda_{a}\right\} \gamma_{5} q .
$$

However, the consequences of the spontaneous symmetry violation can still be analyzed systematically because the quark masses are small. QCD possesses what is called an approximate chiral symmetry. In that case, the mass spectrum of the unperturbed Hamiltonian and the one including the quark masses can not be significantly different. Stated differently, the effects of the explicit symmetry breaking can be analyzed in perturbation theory. As a consequence, QCD has a remarkable mass gap - the pions (and, to a lesser extent, the kaons and the eta) are much lighter than all other hadrons. To be more specific, consider chiral $S U(2)$. The second formula of Eq. (1.5) is nothing but a Ward-identity (WI) that relates the axial current $A^{\mu}=\bar{d} \gamma^{\mu} \gamma_{5} u$ with the pseudoscalar density $P=\bar{d} i \gamma_{5} u$

$$
\partial_{\mu} A^{\mu}=\left(m_{u}+m_{d}\right) P .
$$

Taking on-shell pion matrix elements of this WI, one arrives at

$$
M_{\pi}^{2}=\left(m_{u}+m_{d}\right) \frac{G_{\pi}}{F_{\pi}},
$$

where the coupling $G_{\pi}$ is given by $\langle 0|P(0)| \pi(p)\rangle=G_{\pi}$. This equation leads to some intriguing consequences: In the chiral limit, the pion mass is exactly zero - in accordance with Goldstone's theorem. More precisely, the ratio $G_{\pi} / F_{\pi}$ is a constant in the chiral limit and the pion mass grows as $\sqrt{m_{u}+m_{d}}$ if the quark masses are turned on.

There is even further symmetry related to the quark mass term. It is observed that hadrons appear in isospin multiplets, characterized by very tiny splittings of the order of a few $\mathrm{MeV}$. These are generated by the small quark mass difference $m_{u}-m_{d}$ and also by electromagnetic effects of the same size (with the notable exception of the charged to neutral pion mass difference that is almost entirely of electromagnetic origin). This can be made more precise: For $m_{u}=m_{d}$, QCD is invariant under $S U(2)$ isospin transformations: $q \rightarrow q^{\prime}=U q$, with $U$ a unitary matrix. In this limit, up and down quarks can not be disentangled as far as the strong interactions are concerned. Rewriting of the QCD quark mass term allows to make the strong isospin violation explicit:

$$
\begin{aligned}
\mathcal{H}_{\mathrm{QCD}}^{\mathrm{SB}} & =m_{u} \bar{u} u+m_{d} \bar{d} d \\
& =\frac{m_{u}+m_{d}}{2}(\bar{u} u+\bar{d} d)+\frac{m_{u}-m_{d}}{2}(\bar{u} u-\bar{d} d)
\end{aligned}
$$

where the first (second) term is an isoscalar (isovector). Extending these considerations to $S U(3)$, one arrives at the eighfold way of Gell-Mann and Ne'eman that played a decisive role in our understanding of the quark structure of the hadrons. The $S U(3)$ flavor symmetry is also an approximate one, but the breaking is much stronger than it is the case for isospin. From this, one can directly infer that the quark mass difference $m_{s}-m_{d}$ must be much bigger than $m_{d}-m_{u}$.

The consequences of these broken symmetries can be analyzed systematically in a suitably tailored effective field theory (EFT), as discussed in more detail below. At this point, it is important to stress that the chiral symmetry of QCD plays a crucial role in determining the longest ranged parts of the nuclear force, which, as we will show, is given by Goldstone boson exchange between two and more nucleons. This was already stressed long ago, see e.g (9) (and references therein) but only with the powerful machinery of chiral effective field theory this connection could be worked out model-independently, as we will show in what follows.

\section{B. Scales in nuclear physics}

To appreciate the complexity related to a theoretical description of the nuclear forces, it is most instructive to briefly discuss the pertinent scales arising in this problem. This can most easily be visualized by looking at the phenomenological central potential between two nucleons, as it appears e.g. in meson-theoretical approaches to the nuclear force, see Fig. 1. The longest range part of the interaction is the one-pion exchange (OPE) that is firmly rooted in QCD's chiral symmetry. Thus, the corresponding natural scale of the nuclear force problem is the Compton wavelength of the pion

$$
\lambda_{\pi}=1 / M_{\pi} \simeq 1.5 \mathrm{fm},
$$




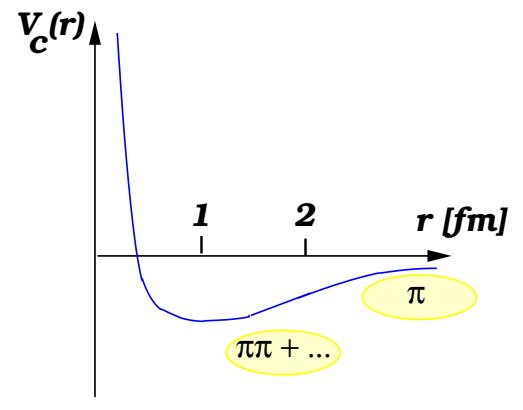

FIG. 1 Schematic plot of the central nucleon-nucleon potential. The longest range contribution is the one-pion-exchange, the intermediate range attraction is described by two-pion exchanges and other shorter ranged contributions. At even shorter distances, the NN interaction is strongly repulsive.

where $M_{\pi}=139.57 \mathrm{MeV}$ is the charged pion mass. The central intermediate range attraction is given by $2 \pi$ exchange (and shorter ranged physics). Finally, the wavefunctions of two nucleons do not like to overlap, which is reflected in a short-range repulsion that can e.g. be modelled by vector meson exchange. From such considerations, one would naively expect to be able to describe nuclear binding in terms of energy scales of the order of the pion mass. However, the true binding energies of the nuclei are given by much smaller energy scales, between 1 to $9 \mathrm{MeV}$ per nucleon. Another measure for the shallow nuclear binding is the so called binding-momentum $\gamma$. In the deuteron, $\gamma=\sqrt{m B_{D}} \simeq 45 \mathrm{MeV} \ll M_{\pi}$, with $m=938.2 \mathrm{MeV}$ the nucleon mass and $B_{D}=2.224 \mathrm{MeV}$ the deuteron binding energy. The small value of $\gamma$ signals the appearance of energy/momentum scales much below the pion mass. The most dramatic reflection of the complexity of the nuclear force problem are the values of the S-wave neutron-proton scattering lengths,

$$
\left|a\left({ }^{1} S_{0}\right)\right|=23.8 \mathrm{fm} \gg 1 / M_{\pi}, a\left({ }^{3} S_{1}\right)=5.4 \mathrm{fm} \gg 1 / M_{\pi} .
$$

Thus, to properly set up an effective field theory for the forces between two (or more) nucleons, it is mandatory to deal with these very different energy scales. If one were to treat the large S-wave scattering lengths perturbatively, the range of the corresponding EFT would be restricted to momenta below $p_{\max } \sim 1 /\left|a\left({ }^{1} S_{0}\right)\right| \simeq 8 \mathrm{MeV}$. To overcome this barrier, one must generate the small binding energy scales by a non-perturbative resummation. This can e.g. be done in a theory without explicit pion degrees of freedom, the so-called pion-less EFT. In such an approach, the limiting hard scale is the pion mass. To go further, one must include the pions explicitely, as it is done in the pion-full or chiral nuclear EFT. The relation between these different approaches is schematically displayed in Fig. 2. A different and more formal argument that shows the breakdown of a perturbative treatment of the EFT with two or more nucleons is related to the pinch singularities in the two-pion exchange diagram in the static limit as will be discussed later in the context

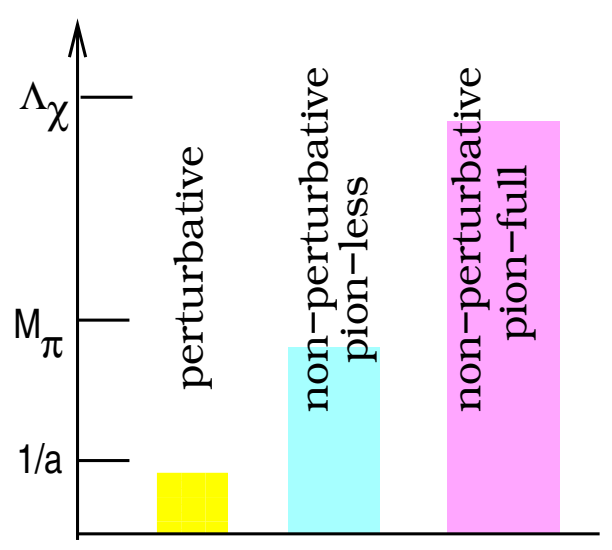

FIG. 2 Scales in the two-nucleon problem and the range of validity of the corresponding EFTs as explained in the text. Here $\Lambda_{\chi}$ is the hard scale related to spontaneous chiral symmetry breaking, with $\Lambda_{\chi} \simeq M_{\rho}$, with $M_{\rho}=770 \mathrm{MeV}$ the mass of the rho meson.

of the explicit construction of the chiral nuclear EFT.

In addition, if one extends the considerations to heavier nuclei or even nuclear matter, the many-body system exhibits yet another scale, the Fermi momentum $k_{F}$, with $k_{F} \simeq 2 M_{\pi}$ at nuclear matter saturation density. This new scale must be included in a properly modified EFT for the nuclear many-body problem which is not a straightforward exercise as we will show below. It is therefore not astonishing that the theory for heavier nuclei is still in a much less developed stage that the one for the few-nucleon problem. These issues will be taken up in Sec. III.

For more extended discussions of scales in the nuclear force problem and in nuclei, we refer to (10-13).

\section{Conventional approaches to the nuclear force problem}

Before discussing the application of the effective field theory approach to the nuclear force problem, let us make a few comments on the highly successful conventional approaches. First, we consider the two-nucleon case. Historically, meson field theory and dispersion relations have laid the foundations for the construction of a twonucleon potential. All these approaches incorporate the long-range one-pion exchange as proposed by Yukawa in 1935 (14) which nowadays is firmly rooted in QCD. Dispersion relations can be used to construct the two-pion exchange contribution to the nuclear force as pioneered at Paris (15) and Stony Brook (16). For a review, see e.g. (17). In the 1990ties, the so-called high-precision potentials have been developed that fit the large basis of $p p$ and $n p$ elastic scattering data with a $\chi^{2} /$ datum $\simeq 1$. One of these is the so-called CD-Bonn potential (18) (which was developed at Moscow, Idaho). Besides one-pion, $\rho$ and $\omega$ vector-meson exchanges, it contains two scalar-isoscalar mesons in each partial wave up to angular momentum 
$J=5$ with the mass and coupling constant of the second $\sigma$ fine-tuned in any partial wave. The hadronic vertices are regulated with form factors with cut-offs ranging from 1.3 to $1.7 \mathrm{GeV}$. Similarly, in the Nijmegen I,II potentials one-pion exchange is supplemented by heavy boson exchanges with adjustable parameters which are fitted for all (low) partial waves separately (19). The Argonne V18 (AV18) potential starts from a very general operator structure in coordinate space and has fit functions for all these various operators (20). While these various potentials give an accurate representation of the nucleonnucleon phase shifts and of most deuteron properties, the situation becomes much less satisfactory when it comes to the much smaller but necessary three-nucleon forces. Such three-body forces are needed to describe the nuclear binding energies and levels, as most systematically shown by the Urbana-Argonne group (21). Systematic studies of the dynamics and reactions of systems with three or four-nucleons further sharpen the case for the necessity of including three-nucleon forces (3NFs), see e.g. (22). The archetype of a $3 \mathrm{NF}$ is due to Fujita and Miyazawa (FM) (23), who extended Yukawa's meson exchange idea by sandwiching the pion-nucleon scattering amplitude between nucleon lines, thus generating the $3 \mathrm{NF}$ of longest range. In fact, the work of Fujita and Miyazawa has been the seed for many meson-theoretical approaches to the three-nucleon force like the families of Tucson-Melbourne (24; 25), Brazilian (26) or Urbana-Illinois (27; 28) 3NFs.

While the conventional approach as briefly outlined here as enjoyed many successes and is frequently used in e.g. nuclear structure and reaction calculations, it remains incomplete as there are certain deficiencies that can only be overcome based on EFT approaches. These are: (i) it is very difficult - if not impossible - to assign a trustworthy theoretical error, (ii) gauge and chiral symmetries are difficult to implement, (iii) none of the three-nucleon forces is consistent with the underlying nucleon-nucleon interaction models/approaches and (iv) the connection to QCD is not at all obvious. Still, as we will show later, there is a very natural connection between these models and the forces derived from EFT by mapping the complicated physics of the shortdistance part of any interaction at length scales $\sim 1 / M_{\rho}$ to the tower of multi-fermion contact interactions that naturally arise in the EFT description (see Sec. II.B).

\section{Brief introduction to effective field theory}

Effective field theory (EFT) is a general approach to calculate the low-energy behavior of physical systems by exploiting a separation of scales in the system (for reviews see e.g. (29-31)). Its roots can be traced to the renormalization group (32) and the intuitive understanding of ultraviolet divergences in quantum field theory (33). A succinct formulation of the underlying principle was given by Weinberg (34): If one starts from the most general Lagrangian consistent with all symmetries of the under-

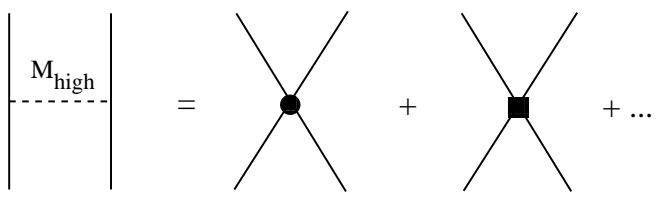

FIG. 3 Expansion of a heavy-particle exchange diagram in terms of local light-particle operators. The solid and dashed lines denote light and heavy particles, respectively. The filled circle and square denote insertions with zero and two derivatives, in order. The ellipses stands for operators with more derivatives.

lying interaction, one will get the most general S-matrix consistent with these symmetries. Together with a power counting scheme that specifies which terms are required at a desired accuracy leads to a predictive paradigm for a low-energy theory. The expansion is typically in powers of a low-momentum scale $M_{\text {low }}$ which can be the typical external momentum over a high-momentum scale $M_{\text {high }}$. However, what physical scales $M_{\text {high }}$ and $M_{\text {low }}$ are identified with depends on the considered system. In its most simple setting, consider a theory that is made of two particle species, the light and the heavy ones with $M_{\text {low }} \ll M_{\text {high }}$. Consider now soft processes in which the energies and momenta are of the order of the light particle mass (the so-called soft scale). Under such conditions, the short-distance physics related to the heavy particles can never be resolved. However, it can be represented by light-particle contact interactions with increasing dimension (number of derivatives). Consider e.g. heavy particle exchange between light ones in the limit that $M_{\text {high }} \rightarrow \infty$ while keeping the ratio $g / M_{\text {high }}$ fixed, with $g$ the light-heavy coupling constant. As depicted in Fig. 3, one can represent such exchange diagrams by a sum of local operators of the light fields with increasing number of derivatives. In a highly symbolic notation

$$
\frac{g^{2}}{M_{h i g h}^{2}-t}=\frac{g^{2}}{M_{h i g h}^{2}}+\frac{g^{2} t}{M_{h i g h}^{4}}+\ldots
$$

with $t$ the squared invariant momentum transfer. In many cases, the corresponding high-energy theory is not known. Still, the framework of EFT offers a predictive and systematic framework for performing calculations in the light particle sector. Denote by $\mathcal{Q}$ a typical energy or momentum of the order of $M_{\text {low }}$ and by $\Lambda$ the hard scale where the EFT will break down. In many cases, this scale is set by the masses of the heavy particles not considered explicitely. In such a setting, any matrix element or Greens function admits an expansion in the small parameter $\mathcal{Q} / \Lambda(34)$

$$
\mathcal{M}=\sum_{\nu}\left(\frac{\mathcal{Q}}{\Lambda}\right)^{\nu} \mathcal{F}\left(\frac{\mathcal{Q}}{\mu}, g_{i}\right)
$$

where $\mathcal{F}$ is a function of order one (naturalness), $\mu$ a regularization scale (related to the UV divergences appearing in the loop graphs) and the $g_{i}$ denotes a collection 
of coupling constants, often called low-energy constants (LECs). These parameterize (encode) the unknown highenergy (short-distance) physics and must be determined by a fit to data (or can be directly calculated if the corresponding high-energy theory is known/can be solved). The counting index $\nu$ in general depends on the fields in the effective theory, the number of derivatives and the number of loops. This defines the so-called power counting which allows to categorize all contributions to any matrix element at a given order. It is important to stress that $\nu$ must be bounded from below to define a sensible EFT. In QCD e.g. this is a consequence of the spontaneous breaking of its chiral symmetry. The contributions with the lowest possible value of $\nu$ define the so-called leading order (LO) contribution, the first corrections with the second smallest allowed value of $\nu$ the next-to-leading order (NLO) terms and so on. In contrast to more conventional perturbation theory, the small parameter is not a coupling constant (like, e.g., in Quantum Electrodynamcis) but rather one expands in small energies or momentum, where small refers to the hard scale $\Lambda$. The archetype of such a perturbative EFT is chiral perturbation theory that exploits the strictures of the spontaneous and explicit chiral symmetry breaking in QCD $(35 ; 36)$. Here, the light degrees of freedom are the pions, that are generated through the symmetry violation. Heavier particles like e.g. vector mesons only appear indirectly as they generate local four-pion interactions with four, six, ... derivatives. For a recent review, see Ref. (37). Of course, the pions also couple to heavy matter fields like e.g. nucleons, that can also be included in CHPT, as reviewed by Bernard (38).

So far, we have made the implicit assumption of naturalness, which implies e.g. that the scattering length $a$ is of natural size as e.g. in CHPT, where the scale is set by $1 / \Lambda_{\chi} \simeq 1 \mathrm{GeV}^{-1} \simeq 0.2 \mathrm{fm}$. This also implies that there are no bound states close to the scattering thresholds. In many physical systems and of particular interest here, especially in the two-nucleon system, this is not the case, but one rather has to deal with unnaturally large scattering lengths (and also shallow bound states). To be specific, let us consider nucleon-nucleon scattering at very low energies in the ${ }^{1} S_{0}$ channel, cf. Eq. (1.10). For such low energies, even the pions can be considered heavy and are thus integrated out. To construct an EFT that is applicable for momenta $p>1 / a$, one must retain all terms $a p \sim 1$ in the scattering matrix. This requires a non-perturbative resummation and is most elegantly done in the power divergence scheme of Kaplan, Savage and Wise $(39 ; 40)$. This amounts to summing the leading four-nucleon contact term $\sim C_{0}\left(\psi^{\dagger} \psi\right)^{2}$ to all orders in $C_{0}$ and matching the scale-dependent LEC $C_{0}$ to the scattering length. This leads to the T-matrix

$$
T=\frac{4 \pi}{m} \frac{1}{1 / a+i p}\left[1+\mathcal{O}\left(p^{2}\right)\right]
$$

where the expansion around the large scattering length is made explicit. All other effects, like e.g. effective range corrections, are treated perturbatively. This compact and elegant scheme is, however, not sufficient for discussing nuclear processes with momenta $p \geq M_{\pi}$. We will come back to this topic when we give the explicit construction of the chiral nuclear EFT in sec. II.B. It is important to stress that such EFTs with unnaturally large scattering length can exhibit universal phenomena that can be observed in physical systems which differ in their typical energy scale by many orders of magnitude, for a review see Braaten and Hammer (41). We also remark that there are many subtleties in constructing a proper EFT, but space forbids to discuss these here. Whenever appropriate and/or neccessary, we will mention these in the following sections and provide explicit references.

\section{E. First results from lattice $Q C D$}

Lattice QCD (LQCD) is a promising tool to calculate hadron properties ab initio from the QCD Lagrangian on a discretized Euclidean space-time. This requires stateof-the-art high performance computers and refined algorithms to analyse the QCD partition function by Monte Carlo methods. Only recently soft- and hardware developments have become available that allow for full QCD simulations at small enough quark masses (corresponding to pion masses below $300 \mathrm{MeV}$ ), large enough volumes (corresponding to spatial dimensions larger than $2.5 \mathrm{fm})$ and sufficiently fine lattice spacing $(a \simeq 0.05 \mathrm{fm})$ so that the results are not heavily polluted by computational artefacts and can really be connected to the physical quark masses by sensible chiral extrapolations.

For the nuclear force problem, there are two main developments in LQCD to be reviewed here. These concern the extraction of hadron-hadron scattering lengths from unquenched simulations and the first attempts to construct a nuclear potential. These are groundbreaking studies, but clearly at present one has not yet achieved an accuracy to obtain high-precision predictions for nuclear properties. We look very much forward to the development of these approaches in the years to come.

The first exploratory study of the nucleon-nucleon scattering lengths goes back to Fukugita et al. $(42 ; 43)$ in the quenched approximation. They make use of an elegant formuala, frequently called the "Lüscher formula", that relates the S-wave scattering length $a_{0}$ between two hadrons $h_{1}$ and $h_{2}$ to the energy shift $\delta E=$ $E_{h_{1} h_{2}}-\left(m_{1}+m_{2}\right)$ of the two-hadron state at zero relative momentum confined in spatial box of size $L^{3}$. It is given by (44-46)

$$
\delta E=-\frac{2 \pi a_{0}}{\mu L^{3}}\left[1+c_{1} \frac{a_{0}}{L}+c_{2} \frac{a_{0}^{2}}{L^{2}}\right]+\mathcal{O}\left(L^{-6}\right),
$$

with $\mu=m_{1} m_{2} /\left(m_{1}+m_{2}\right)$ the reduced mass and $c_{1}=$ 2.837297 and $c_{2}=6.375183$. A generalization of this formalism was given by Beane et al.(47) utilizing methods developed for the so-called pionless nuclear EFT (EFT 
with contact interactions, for a review see e.g. (48)). It reads

$$
\begin{aligned}
p \cot \delta_{0}(p) & =\frac{1}{\pi L} \mathcal{S}\left((L p / 2 \pi)^{2}\right), \\
\mathcal{S}(\eta) & =\sum_{\vec{j}}^{\Lambda_{j}} \frac{1}{|\vec{j}|^{2}-\eta}-4 \pi \Lambda_{j},
\end{aligned}
$$

which gives the location of all energy eigenstates in the box. Here, $\delta_{0}$ is the S-wave phase shift. The sum over all three-vectors of integers $\vec{j}$ is such that $|\vec{j}|<$ $\Lambda_{j}$ and the limit $\Lambda_{j} \rightarrow \infty$ is implicit. In the limit $L \gg\left|a_{0}\right|$, Eq. (1.15) reduces to the Lüscher formula, Eq. (1.14). On the other hand, for large scattering length, $\left|\left(p \cot \delta_{0}\right)^{-1}\right| \gg L$, the energy of the lowest state is given by

$$
E_{0}=\frac{4 \pi^{2}}{\mu L^{2}}\left[d_{1}+d_{2} L p \cot \delta_{0}+\ldots\right],
$$

with $d_{1}=0.472895, d_{2}=0.0790234$ and $p \cot \delta_{0}$ is evaluated at the energy $E=4 \pi^{2} d_{1} /\left(\mu L^{2}\right)$. Within this framework, in Ref. (49) the first fully dynamical simulation of the neutron-proton scattering lengths was performed, with a lowest pion mass of $354 \mathrm{MeV}$. This mass is still too high to perform a precise chiral extrapolation to the physical pion mass, but this calculation clearly demonstrates the feasilbilty of this approach (see also sec. II.H). This scheme can also be extended to hyperon-nucleon interactions, see (50). A first signal for $\pi \Lambda$ and $n \Sigma^{-}$scattering was reported in Ref. (51). For a recent review on these activities of the NPLQCD collaboration, we refer the reader to (52).

Another interesting development was initiated and carried out by Aoki, Hatsuda and Ishii (53). They have generalized the two-center Bethe-Salpeter wavefunction approach of the CP-PACS collaboration (54), which offers an alternative to Lüscher's formula, to the two-nucleon (NN) system. Given an interpolating field for the neutron and for the proton, the NN potential can be defined from the properly reduced 6-quark Bethe-Salpeter amplitude $\phi(\vec{r})$. The resulting Lippmann-Schwinger equation defines a non-local potential for a given, fixed separation $r=|\vec{r}|$. Performing a derivative expansion, the central potential $V_{c}(r)$ at a given energy $E$ is extracted from

$$
V_{C}(r)=E+\frac{1}{m} \frac{\vec{\nabla}^{2} \phi(r)}{\phi(r)} .
$$

Monte Carlo Simulations are then performed to generate the 6-quark Bethe-Salpeter amplitude in a given spin and isospin state of the two-nucleon system on a large lattice, $V=(4.4 \mathrm{fm})^{4}$ in the quenched approximation for pion masses between 380 and $730 \mathrm{MeV}$. Despite these approximations, the resulting effective potential extracted using Eq. (1.17) shares the features of the phenomenological NN potentials - a hard core (repulsion) at small separation surrounded by an attractive well at intermediate and larger distances, see Fig. 4. Furthermore, the

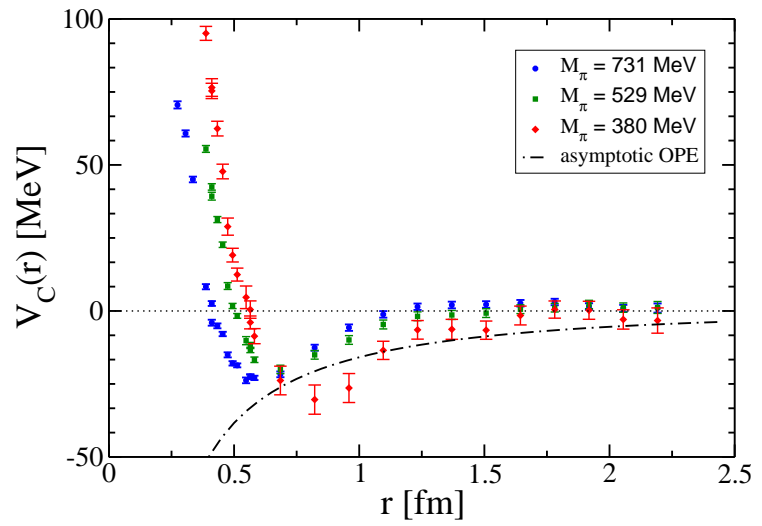

FIG. 4 Effective potential in the ${ }^{1} S_{0}$ channel for three different quark masses in quenched LQCD after Ref. (55). The dashed line is the asymptotic OPEP for $M_{\pi}=380 \mathrm{MeV}$, $m=1.2 \mathrm{GeV}$ and $g_{i N}^{2} /(4 \pi)=14.0$. We are grateful to Dr. N. Ishii for providing us with the data.

asymptotic form of this potential has exactly the form of the OPE, provided one rescales the formula

$$
V_{C}^{\mathrm{OPE}}(r)=\frac{g_{\pi N}^{2}}{4 \pi} \boldsymbol{\tau}_{1} \cdot \boldsymbol{\tau}_{2} \frac{\vec{\sigma}_{1} \cdot \vec{\sigma}_{2}}{3}\left(\frac{M_{\pi}}{2 m}\right)^{2} \frac{\mathrm{e}^{-M_{\pi} r}}{r}
$$

with the pion and nucleon masses used in the simulations but keeping the pion-nucleon coupling at its physical value, $g_{\pi N}^{2} /(4 \pi) \simeq 14.0$. These interesting results have led to some enthusiastic appraisal, see e.g. (56). However, it is important to stress that the so-calculated potential is not unique, especially its properties at short distances, since it depends on the definition of the interpolating nucleon fields. Furthermore, the quenched approximation is known to have uncontrolled systematic uncertainties as it does not even define a quantum field theory. In this context, the authors of Ref. (54) report on the numerical absence of the large-distancedominating $\eta$-exchange from the flavor-singlet hair-pin diagram. Still, one would like to see this promising calculation repeated with dynamical quarks of sufficiently small masses. In Ref. (57) this framework was used to study the $\Xi N$ interaction. Interestingly, the central potential of the $p \Xi^{0}$ interaction looks very similar to the central $n p$ potential. It would be interesting to extend these calculations to other hyperon-nucleon channels and also study the effects of SU(3) symmetry breaking. We will come back to these issues in the context of an threeflavor chiral EFT in sec. II.F. For recent developments in this scheme concerning the calculation of the tensor force, the energy dependence of the NN potential and preliminary results for full QCD (2+1 flavors), see the talks by Aoki, Ishii, and Nemura at the Lattice 2008 conference (58). 


\section{F. Observables and not-so observable quantities}

There is an extensive literature, primarily from the sixties and seventies, on the role of off-shell physics in nuclear phenomena (see, e.g., Ref. (59) and references therein). This includes not only few-body systems (e.g., the triton) and nuclear matter, but interactions of twobody systems with external probes, such as nucleonnucleon bremstrahlung and the electromagnetic form factors of the deuteron. The implicit premise was that there is a true underlying potential governing the nucleonnucleon force, so that its off-shell properties can be determined. Indeed, the nuclear many-body problem has traditionally been posed as finding approximate solutions to the many-particle Schrödinger equation, given a fundamental two-body interaction that reproduces two-nucleon observables.

In contrast, effective field theories are determined completely by on-energy-shell information, up to a welldefined truncation error. In writing down the most general Lagrangian consistent with the symmetries of the underlying theory, many-body forces arise naturally. Even though they are usually suppressed at low energies, they enter at some order in the EFT expansion. These manybody forces have to be determined from many-body data. The key point is, however, that no off-energy-shell information is needed or experimentally accessible. A fundamental theorem of quantum field theory states that physical observables (or more precisely, S-matrix elements) are independent of the choice of interpolating fields that appear in a Lagrangian $(60 ; 61)$. Equivalently, observables are invariant under a change of field variables in a field theory Lagrangian (or Hamiltonian):

$$
\psi(x) \rightarrow \psi(x)+\eta P[\psi],
$$

where $P[\psi]$ is a local polynomial of the field $\psi$ and its derivatives and $\eta$ is an arbitray counting parameter. Newly generated contributions to observables have to cancel separately at each order in $\eta$. This "equivalence theorem" holds for renormalized field theories. In an EFT, one exploits the invariance under field redefinitions to eliminate redundant terms in the effective Lagrangian and to choose the most convenient or efficient form for practical calculations (62-66). Since off-shell Green's functions and the corresponding off-shell amplitudes do change under field redefinitions, one must conclude that off-shell properties are unobservable.

Several recent works have emphasized from a field theory point of view the impossibility of observing offshell effects. In Refs. $(67 ; 68)$, model calculations were used to illustrate how apparent determinations of the two-nucleon off-shell T-matrix in nucleon-nucleon bremstrahlung are illusory, since field redefinitions shift contributions between off-shell contributions and contact interactions. Similarly, it was shown in Ref. (69) that Compton scattering on a pion cannot be used to extract information on the off-shell behavior of the pion form factor. The authors of Refs. (70; 71) emphasized the nonunique- ness of chiral Lagrangians for three-nucleon forces and pion production. Field redefinitions lead to different offshell forms that yield the same observables within a consistent power counting. In Ref. (72), an interaction proportional to the equation of motion is shown to have no observable consequence for the deuteron electromagnetic form factor, even though it contributes to the off-shell T-matrix.

In systems with more than two nucleons, one can trade off-shell, two-body interactions for many-body forces. This explains how two-body interactions related by unitary transformations can predict different binding energies for the triton (73) if many-body forces are not consistently included. These issues were discussed from the viewpoint of unitary transformations in Refs. (74) and (75). The extension to many-fermion systems in the thermodynamic limit was considered in (76). The effects of field redefinitions were illustrated using the EFT for the dilute Fermi gas (77). If many-body interactions generated by the field redefinitions are neglected, a Coester line similar to the one observed for nuclear matter (78) is generated. Moreover, the connection to more traditional treatments using unitary transformations was elucidated. The question of whether occupation numbers and momentum distributions of nucleons in nuclei are observables was investigated in Ref. (79). Field redefinitions lead to variations in the occupation numbers and momentum distributions that imply the answer is negative. The natural size of the inherent ambiguity (or scheme dependence) in these quantities is determined by the applicability of the impulse approximation. Only if the impulse approximation is well justified, the ambiguity is small and these quantities are approximately scheme independent. This has important implications for the interpretation of $\left(e, e^{\prime} p\right)$ experiments with nuclei. Whether the stark difference in occupation numbers between nonrelativistic and relativistic Brueckner calculations can be explained by this ambiguity is another interesting question (80).

\section{EFT FOR FEW-NUCLEON SYSTEMS: FOUNDATIONS AND APPLICATIONS}

\section{A. EFT with contact interactions and universal aspects}

In nuclear physics, there are a number of EFTs which are all useful for a certain range of systems (cf. Fig. 2). The simplest theories include only short range interactions and even integrate out the pions. At extremely low energies, $M_{h i g h}$ is given by the NN scattering lengths and one can formulate a perturbative EFT in powers of of the typical momentum $k$ divided by $M_{h i g h}$. Since the NN scattering lengths are large this theory has a very limited range of applicability. It is therefore useful to construct another EFT with short-range interactions that resums the interactions generating the large scattering length. This so-called pionless EFT can be understood as an ex- 
pansion around the limit of infinite scattering length or equivalently around threshold bound states. Its breakdown scale is set by one-pion exchange, $M_{\text {high }} \sim M_{\pi}$, while $M_{\text {low }} \sim 1 / a \sim k$. For momenta $k$ of the order of the pion mass $M_{\pi}$, pion exchange becomes a long-range interaction and has to be treated explicitly. This leads to the chiral EFT whose breakdown scale $M_{h i g h}$ is set by the chiral symmetry breaking scale $\Lambda_{\chi}$ and will be discussed in detail below.

The pionless theory relies only on the large scattering length and is independent of the mechanism responsible for it. It is very general and can be applied in systems ranging from ultracold atoms to nuclear and particle physics. It is therefore ideally suited to unravel universal phenomena driven by the large scattering length such as limit cycle physics $(81 ; 82)$ and the Efimov effect (83). For recent reviews of applications to the physics of ultracold atoms, see Refs. $(41 ; 84)$. Here we consider applications of this theory in nuclear physics.

The pionless EFT is designed to reproduce the well known effective range expansion. The leading order Lagrangian can be written as:

$$
\begin{aligned}
\mathcal{L} & =N^{\dagger}\left(i \partial_{0}+\frac{\vec{\nabla}^{2}}{2 m}\right) N \\
& -C_{0}^{t}\left(N^{T} \tau_{2} \sigma_{i} \sigma_{2} N\right)^{\dagger}\left(N^{T} \tau_{2} \sigma_{i} \sigma_{2} N\right) \\
& -C_{0}^{s}\left(N^{T} \sigma_{2} \tau_{a} \tau_{2} N\right)^{\dagger}\left(N^{T} \sigma_{2} \tau_{a} \tau_{2} N\right)+\ldots,
\end{aligned}
$$

where the dots represent higher-order terms suppressed by derivatives and more nucleon fields. The Pauli matrices $\sigma_{i}\left(\tau_{a}\right)$ operate in spin (isospin) space, respectively. The contact terms proportional to $C_{0}^{t}\left(C_{0}^{s}\right)$ correspond to two-nucleon interactions in the ${ }^{3} \mathrm{~S}_{1}\left({ }^{1} \mathrm{~S}_{0}\right) \mathrm{NN}$ channels. Their renormalized values are related to the corresponding large scattering lengths $a_{t}$ and $a_{s}$ in the spin-triplet and spin-singlet channels, respectively. The exact relation, of course, depends on the renormalization scheme. Various schemes can be used, such as a momentum cutoff or dimensional regularization. Convenient schemes that have a manifest power counting at the level of individual diagrams are dimensional regularization with PDS subtraction, where poles in 2 and 3 spatial dimensions are subtracted (40), or momentum subtractions schemes as in (85). However, a simple momentum cutoff can be used as well.

Since the scattering lengths are set by the lowmomentum scale $a \sim 1 / M_{\text {low }}$, the leading contact interactions have to be resummed to all orders $(40 ; 86)$. The nucleon-nucleon scattering amplitude in the ${ }^{3} \mathrm{~S}_{1}$ $\left({ }^{1} \mathrm{~S}_{0}\right)$ channels is obtained by summing the so-called bubble diagrams with the $C_{0}^{t}\left(C_{0}^{s}\right)$ interactions shown in Fig. 5. This summation gives the exact solution of the Lippmann-Schwinger equation for the $C_{0}^{t}$ or $C_{0}^{s}$ interactions. Higher order derivative terms which are not shown explicitly in Eq. (2.1) reproduce higher order terms in the effective range expansion. Since these terms are natural and their size is set by $M_{h i g h}$, their contribution at

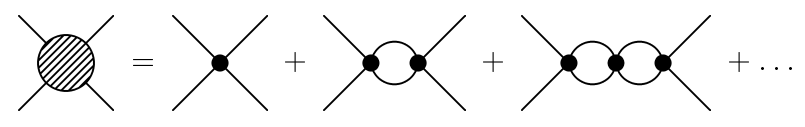

FIG. 5 The bubble diagrams with the contact interaction $C_{0}^{t}$ or $C_{0}^{s}$ contributing to the two-nucleon scattering amplitude.

low energies is suppressed by powers of $M_{l o w} / M_{\text {high }}$ and can be treated in perturbation theory. The subleading correction is given by the effective range $r_{0} \sim 1 / M_{\text {high }}$ and the corresponding diagrams are illustrated in Fig. 6. The renormalized S-wave scattering amplitude to nextto-leading order in a given channel then takes the form

$$
T_{2}(k)=\frac{4 \pi}{m} \frac{1}{-1 / a-i k}\left[1-\frac{r_{0} k^{2} / 2}{-1 / a-i k}+\ldots\right]
$$

where $k$ is the relative momentum of the nucleons and the dots indicate corrections of order $\left(M_{\text {low }} / M_{\text {high }}\right)^{2}$ for typical momenta $k \sim M_{\text {low }}$. The pionless EFT becomes very useful in the two-nucleon sector when external currents are considered and has been applied to a variety of electroweak processes. These calculations are reviewed in detail in Refs. (48; 87). More recently Christlmeier and Grießhammer have calculated low-energy deuteron electrodisintegration in the framework of the pionless EFT (88). For the double differential cross sections of the $d\left(e, e^{\prime}\right)$ reaction at $\theta=180^{\circ}$ excellent agreement was found with a recent experiment at S-DALINAC (89).\#1 The double-differential cross section for an incident electron energy $E_{0}=27.8 \mathrm{MeV}$ and $\theta=180^{\circ}$ is shown in Fig. 7. The data were used to precisely map the $M 1$ response which governs the reaction $n p \rightarrow d \gamma$ relevant to big-bang nucleosythesis. Finally, the reaction $p p \rightarrow p p \pi^{0}$ near threshold was studied by Ando (90).

We now proceed to the three-nucleon system. Here it is convenient (but not mandatory) to rewrite the theory using so-called "dimeron" auxilliary fields (91). We need two dimeron fields, one for each S-wave channel: (i) a field $t_{i}$ with spin (isospin) 1 (0) representing two nucleons interacting in the ${ }^{3} S_{1}$ channel (the deuteron) and (ii) a field $s_{a}$ with spin (isospin) 0 (1) representing two nucleons

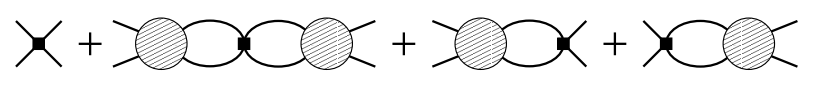

FIG. 6 Diagrams for the inclusion of higher order contact interactions.

\#1 However, there is a disagreement between theory and data for the small longitudinal-transverse interference contribution $\sigma_{L T}$ that is currently not understood (88). 


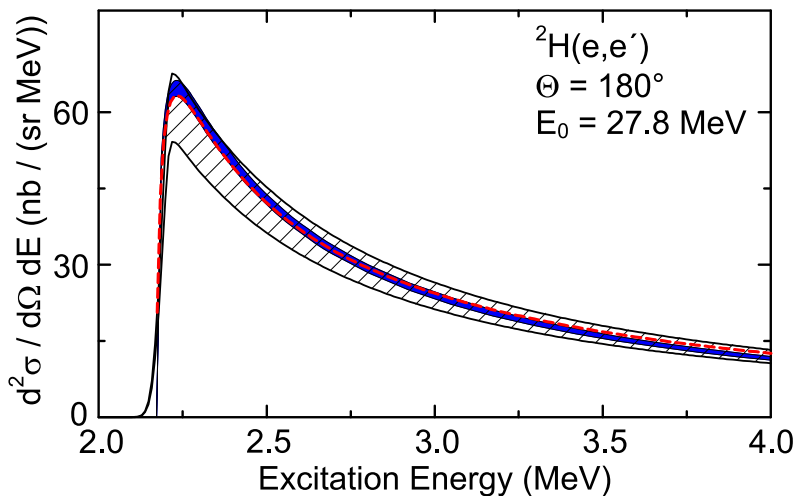

FIG. 7 Double-differential cross sections of the ${ }^{2} \mathrm{H}\left(e, e^{\prime}\right)$ reaction with errors (hatched bands) extracted from the experiment. The gray bands and dashed lines are calculations in pionless EFT and a potential model. Figure courtesy of H.W. Grießhammer.

interacting in the ${ }^{1} S_{0}$ channel (92):

$$
\begin{aligned}
\mathcal{L} & =N^{\dagger}\left(i \partial_{t}+\frac{\vec{\nabla}^{2}}{2 m}\right) N-t_{i}^{\dagger}\left(i \partial_{t}-\frac{\vec{\nabla}^{2}}{4 m}-\Delta_{t}\right) t_{i} \\
& -s_{a}^{\dagger}\left(i \partial_{t}-\frac{\vec{\nabla}^{2}}{4 m}-\Delta_{s}\right) s_{a}-\frac{g_{t}}{2}\left(t_{i}^{\dagger} N^{T} \tau_{2} \sigma_{i} \sigma_{2} N+\text { h.c. }\right) \\
& -\frac{g_{s}}{2}\left(s_{a}^{\dagger} N^{T} \sigma_{2} \tau_{a} \tau_{2} N+\text { h.c. }\right)-G_{3} N^{\dagger}\left[g_{t}^{2}\left(t_{i} \sigma_{i}\right)^{\dagger}\left(t_{j} \sigma_{j}\right)\right. \\
& \left.+\frac{g_{t} g_{s}}{3}\left(\left(t_{i} \sigma_{i}\right)^{\dagger}\left(s_{a} \tau_{a}\right)+\text { h.c. }\right)+g_{s}^{2}\left(s_{a} \tau_{a}\right)^{\dagger}\left(s_{b} \tau_{b}\right)\right] N \\
& +\ldots,
\end{aligned}
$$

where $i, j$ are spin and $a, b$ are isospin indices while $g_{t}$, $g_{s}, \Delta_{t}, \Delta_{s}$ and $G_{3}$ are the bare coupling constants. This Lagrangian goes beyond leading order and already includes the effective range terms. The coupling constants $g_{t}, \Delta_{t}, g_{s}, \Delta_{s}$ are matched to the scattering lengths $a_{\alpha}$ and effective ranges $r_{0 \alpha}$ in the two channels $(\alpha=s, t)$. Alternatively, one can match to the position of the bound state/virtual state pole $\gamma_{\alpha}$ in the $T$-matrix instead of the scattering length which often improves convergence (93). The two quantities are related through:

$$
\gamma_{\alpha}=\frac{1}{r_{0 \alpha}}\left(1-\sqrt{1-2 r_{0 \alpha} / a_{\alpha}}\right)
$$

where $\alpha=s, t$. The term proportional to $G_{3}$ constitutes a Wigner- $S U(4)$ symmetric three-body interaction. It only contributes in the spin-doublet S-wave channel. When the auxilliary dimeron fields $t_{i}$ and $s_{a}$ are integrated out, an equivalent form containing only nucleon fields is obtained. At leading order when the effective range corrections are neglected, the spatial and time derivatives acting on the dimeron fields are omitted and the field is static. The coupling constants $g_{\alpha}$ and $\Delta_{\alpha}$, $\alpha=s, t$ are then not independent and only the combination $g_{\alpha}^{2} / \Delta_{\alpha}$ enters in observables. This combination can then be matched to the scattering length or pole position.

The simplest three-body process to consider is neutron-deuteron scattering below the breakup thresh-

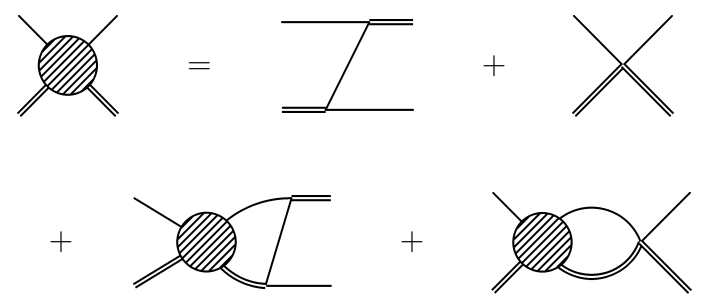

FIG. 8 The integral equation for the boson-dimeron scattering amplitude. The single (double) line indicates the boson (dimeron) propagator.

old. In order to focus on the main aspects of renormalization, we suppress all spin-isospin indices and complications from coupled channels in the three-nucleon problem. This corresponds to a system of three spinless bosons with large scattering length. If the scattering length is positive, the bosons form a two-body bound state analog to the deuteron which we call dimeron. The leading order integral equation for boson-dimeron scattering is shown schematically in Fig. 8. For total orbital angular momentum $L=0$, it takes the following form:

$$
\begin{aligned}
T_{3}(k, p ; E)= & \frac{16}{3 a} M(k, p ; E)+\frac{4}{\pi} \int_{0}^{\Lambda} d q q^{2} T_{3}(k, q ; E) \\
& \times \frac{M(q, p ; E)}{-1 / a+\sqrt{3 q^{2} / 4-m E-i \epsilon}},
\end{aligned}
$$

where the inhomogeneous term reads

$$
M(k, p ; E)=\frac{1}{2 k p} \ln \left(\frac{k^{2}+k p+p^{2}-m E}{k^{2}-k p+p^{2}-m E}\right)+\frac{H(\Lambda)}{\Lambda^{2}} .
$$

Here, $H$ determines the strength of the three-body force $G_{3}(\Lambda)=2 m H(\Lambda) / \Lambda^{2}$ which enters already at leading order and $\Lambda$ is a UV cutoff introduced to regularize the integral equation. The magnitude of the incoming (outgoing) relative momenta is $k(p)$ and $E=3 k^{2} /(4 m)-1 /\left(m a^{2}\right)$. The on-shell point corresponds to $k=p$ and the phase shift can be obtained via $k \cot \delta=1 / T_{3}(k, k ; E)+i k$. For $H=0$ and $\Lambda \rightarrow \infty$, Eq. (2.5) reduces to the STM equation first derived by Skorniakov and Ter-Martirosian (94). It is well known that the STM equation has no unique solution (95). The regularized equation has a unique solution for any given (finite) value of the ultraviolet cutoff $\Lambda$ but the amplitude in the absence of the three-body force shows an oscillatory behavior on $\ln \Lambda$. Cutoff independence of the amplitude is restored by an appropriate "running" of $H(\Lambda)$ which turns out to be a limit cycle (96; 97):

$$
H(\Lambda)=\frac{\cos \left[s_{0} \ln \left(\Lambda / \Lambda_{*}\right)+\arctan s_{0}\right]}{\cos \left[s_{0} \ln \left(\Lambda / \Lambda_{*}\right)-\arctan s_{0}\right]}
$$

where $\Lambda_{*}$ is a dimensionful three-body parameter generated by dimensional transmutation. Adjusting $\Lambda_{*}$ to a single three-body observable allows to determine all other 
low-energy properties of the three-body system. Note that the choice of the three-body parameter $\Lambda_{*}$ is not unique and there are other definitions more directly related to experiment (41). Because $H(\Lambda)$ in Eq. (2.7) vanishes for certain values of the cutoff $\Lambda$ it is possible to eliminate the explicit three-body force from the equations by working with a fixed cutoff that encodes the dependence on $\Lambda_{*}$. This justifies tuning the cutoff $\Lambda$ in the STM equation to reproduce a three-body datum and using the same cutoff to calculate other observables as suggested by Kharchenko (98). Equivalently, a subtraction can be performed in the integral equation $(99 ; 100)$. In any case, one three-body input parameter is needed for the calculation of observables. A comprehensive study of the range corrections to the three-boson spectrum was carried out in Ref. (101). The authors showed that all range corrections vanish in the unitary limit due to the discrete scale invariance. While the corrections proportional to $r_{0} / a$ vanish trivially, this includes also the corrections proportional to $\kappa_{*} r_{0}$ where $\kappa_{*}=\sqrt{m B_{3}^{*}}$ is the binding momentum of the Efimov state fixed by the chosen renormalization condition. Moreover, they have calculated the corrections to the Efimov spectrum for finite scattering length. The range corrections are negligible for the shallow states but become important for the deeper bound states.

The integral equations for the three-nucleon problem derived from the Lagrangian (2.3) are a generalization of Eq. (2.5). (For their explicit form and derivation, see e.g. Ref. (102).) For S-wave nucleon-deuteron scattering in the spin-quartet channel only the spin-1 dimeron field contributes. This integral equation has a unique solution for $\Lambda \rightarrow \infty$ and there is no three-body force in the first few orders. The spin-quartet scattering phases can therefore be predicted to high precision from twobody data $(103 ; 104)$. In the spin-doublet channel both dimeron fields as well as the the three-body force in the Lagrangian (2.3) contribute (92). This leads to a pair of coupled integral equations for the T-matrix. Thus, one needs a new parameter which is not determined in the $2 \mathrm{~N}$ system in order to fix the (leading) low-energy behavior of the $3 \mathrm{~N}$ system in this channel. The three-body parameter gives a natural explanation of universal correlations between different three-body observables such as the Phillips line, a correlation between the triton binding energy and the spin-doublet neutron-deuteron scattering length (105). These correlations are purely driven by the large scattering length independent of the mechanism responsible for it. As a consequence, they occur in atomic systems such as ${ }^{4} \mathrm{He}$ atoms as well (41).

Higher-order corrections to the amplitude including the ones due to $2 \mathrm{~N}$ effective range terms can be included perturbatively. This was first done at NLO for the scattering length and triton binding energy in (106) and for the energy dependence of the phase shifts in (99). In Refs. $(102 ; 107)$, it was demonstrated that it is convenient to iterate certain higher order range terms in order to extend the calculation to $\mathrm{N}^{2} \mathrm{LO}$. Here, also a sub-

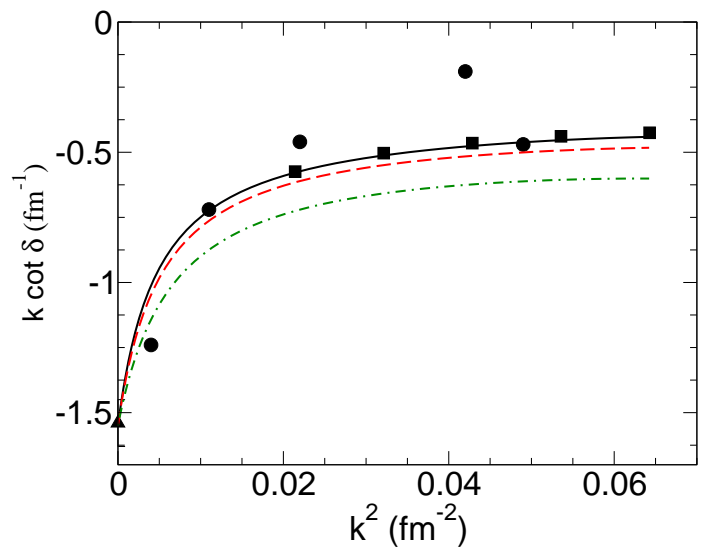

FIG. 9 Phase shifts for neutron-deuteron scattering below the deuteron breakup at LO (dash-dotted line), NLO (dashed line), and $\mathrm{N}^{2} \mathrm{LO}$ (solid line). The filled squares and circles give the results of a phase shift analysis and a calculation using AV18 and the Urbana IX three-body force, respectively. Figure courtesy of L. Platter.

leading three-body force was included as required by dimensional analysis. More recently, Platter and Phillips showed using the subtractive renormalization that the leading three-body force is sufficient to achieve cutoff independence up to $\mathrm{N}^{2} \mathrm{LO}$ in the expansion in $M_{\text {low }} / M_{\text {high }}$ (108). The results for the spin-doublet neutron-deuteron scattering phase shift at LO (92), NLO (99), and $\mathrm{N}^{2} \mathrm{LO}$ (109) are shown in Fig. 9. There is excellent agreement with the available phase shift analysis and a calculation using a phenomenological NN interaction. Whether there is a suppression of the subleading three-body force or simply a correlation between the leading and subleading contributions is not fully understood. The extension to $3 \mathrm{~N}$ channels with higher orbital angular momentum is straightforward (110) and three-body forces do not appear until very high orders. A general counting scheme for three-body forces based on the asymptotic behavior of the solutions of the leading order STM equation was proposed in (111). A complementary approach to the fewnucleon problem is given by the renormalization group where the power counting is determined from the scaling of operators under the renormalization group transformation (32). This method leads to consistent results for the power counting (112-114). Universal low-energy properties of few-body systems with short-range interactions and large two-body scattering length were reviewed in (41). (See also (115) for an early work on this subject.) Three-body calculations with external currents are still in their infancy. However, a few exploratory calculations have been carried out. Universal properties of the triton charge form factor were investigated in Ref. (116) and neutron-deuteron radiative capture was calculated in Refs. $(117 ; 118)$. This opens the possibility to carry out accurate calculations of electroweak reactions at very low energies for astrophysical processes.

The pionless approach has also been extended to the 


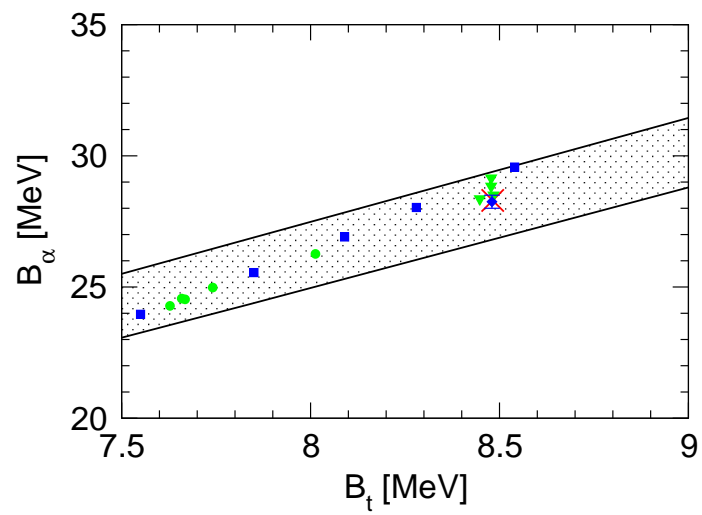

FIG. 10 The Tjon line correlation as predicted by the pionless theory. The grey circles and triangles show various calculations using phenomenological potentials (121). The squares show the results of chiral EFT at NLO for different cutoffs while the diamond gives the $\mathrm{N}^{2} \mathrm{LO}$ result $(122 ; 123)$. The cross shows the experimental point.

four-body sector $(119 ; 120)$. In order to be able to apply the Yakubovsky equations, an equivalent effective quantum mechanics formulation was used. The study of the cutoff dependence of the four-body binding energies revealed that no four-body parameter is required for renormalization at leading order. As a consequence, there are universal correlations in the four-body sector which are also driven by the large scattering length. The best known example is the Tjon line: a correlation between the triton and alpha particle binding energies, $B_{t}$ and $B_{\alpha}$, respectively. Of course, higher order corrections break the exact correlation and generate a band. In Fig. 10, we show this band together with some some calculations using phenomenological potentials (121) and a chiral EFT potential with explicit pions (122; 123). All calculations with interactions that give a large scattering length must lie within the band. Different shortdistance physics and/or cutoff dependence should only move the results along the band. This can for example be observed in the NLO results with the chiral potential indicated by the squares in Fig. 10 or in the few-body calculations with the low-momentum NN potential $V_{\text {low k }}$ carried out in Ref. (124). The $V_{\text {low k potential is obtained }}$ from phenomenological $\mathrm{NN}$ interactions by intergrating out high-momentum modes above a cutoff $\Lambda$ but leaving two-body observables (such as the large scattering lengths) unchanged. The results of few-body calculations with $V_{\text {low k }}$ are not independent of $\Lambda$ but lie all close to the Tjon line (cf. Fig. 2 in Ref. (124)). The studies of the four-body system in the pionless theory were extendend further in Ref. (125). Here the dependence of the four-body bound state spectrum on the two-body scattering length was investigated in detail and summarized in a generalized Efimov plot for the four-body spectrum.

The question of whether a four-body parameter has to enter at leading order was reanalyzed by Yamashita et al. (126). Within the renormalized zero-range model, they found a strong sensitivity of the deepest four-body energy to a four-body subtraction constant in their equations. They motivated this observation from a general model-space reduction of a realistic two-body interaction close to a Feshbach resonance. The results of Ref. (119) for the ${ }^{4} \mathrm{He}$ tetramer that include a four-body parameter were also reproduced. Yamashita et al. concluded that a four-body parameter should generally enter at leading order. They argued that four-body systems of ${ }^{4} \mathrm{He}$ atoms and nucleons (where this sensitivity is absent $(119 ; 120 ; 124)$ ) are special because repulsive interactions strongly reduce the probability to have four particles close together. However, the renormalization of the four-body problem was not explicitly verified in their calculation. Another drawback of their analysis is the focus on the deepest four-body state only. Therefore, their findings could be an artefact of their particular regularization scheme. Another recent study by von Stecher and collaborators (127) confirmed the absence of a four-body parameter for shallow states while some sensitivity was found for the deepest four-body state.

The pionless theory has also been extended to more than four particles using it within the no-core shell model approach. Here the expansion in a truncated harmonic oscillator basis is used as the ultraviolet regulator of the EFT. The effective interaction is determined directly in the model space, where an exact diagonalization in a complete many-body basis is performed. In Ref. (128), the $0^{+}$excited state of ${ }^{4} \mathrm{He}$ and the ${ }^{6} \mathrm{Li}$ ground state were calculated using the deuteron, triton, alpha particle ground states as input. The first $\left(0^{+} ; 0\right)$ excited state in ${ }^{4} \mathrm{He}$ is calculated within $10 \%$ of the experimental value, while the ${ }^{6} \mathrm{Li}$ ground state comes out at about $70 \%$ of the experimental value in agreement with the $30 \%$ error expected for the leading order approximation. These results are promising and should be improved if range corrections are included. Finally, the spectrum of trapped three- and four-fermion systems was calculated using the same method (129). In this case the harmonic potential is physical and not simply used as an ultraviolet regulator.

\section{B. Chiral EFT for few nucleons: foundations}

The extension of the previously discussed EFT with contact interactions to higher energies requires the inclusion of pions as explicit degrees of freedom. The interaction between pions and nucleons can be described in a systematic way using chiral perturbation theory. In contrast, the interaction between the nucleons is strong and leads to nonperturbative phenomena at low energy such as e.g. shallow-lying bound states. This breakdown of perturbation theory can be linked to the fact that the interaction between the nucleons is not suppressed in the chiral limit contrary to the pion and pionnucleon interactions. Moreover, an additional enhancement occurs for Feynman diagrams involving two and 


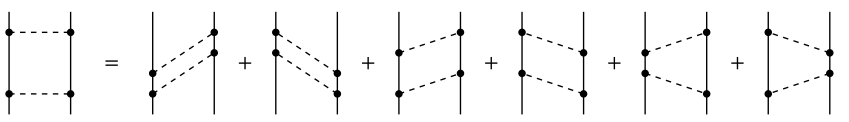

FIG. 11 Representation of the two-pion exchange Feynman diagram in terms of time-ordered graphs. Solid and dashed lines represent nucleons and pions, respectively.

more nucleons due to the appearance of the so-called pinch singularities in the limit of the infinite nucleon mass. Although such infrared singularities disappear if one keeps the nucleon mass at its physical value, they do generate large enhancement factors which destroy the chiral power counting. This can be more easily understood utilizing the language of time-ordered perturbation theory. Consider, for example, the two-pion exchange box diagram shown in Fig. 11. While all intermediate states in the first two time-ordered graphs, often referred to as irreducible, involve at least one virtual pion and thus lead to energy denimonators of the expected size, $E-E_{i} \sim M_{\pi}$, the remaining reducible diagrams involve an intermediate state with nucleons only which produces unnaturally small energy denominators of the order $E-E_{i} \sim M_{\pi}^{2} / m \ll M_{\pi}$. Clearly, the enhanced reducible time-ordered diagrams are nothing but the iterations of the Lippmann-Schwinger equation with the kernel which contains all possible irreducible diagrams and defines the nuclear Hamiltonian. It is free from infrared enhancement factors and can be worked out systematically using the machinery of chiral perturbation theory as suggested in Weinberg's seminal work $(130 ; 131)$ \#2 This natural reduction to the quantum mechanical $A$-body problem is a welcome feature for practical calculations as it allows to apply various existing few-body techniques such as e.g. the Faddeev-Yakubovsky scheme, the no-core shell model, Green's function Monte Carlo and hyperspherical harmonics methods. On the other hand, the framework offers a systematic and perturbative scheme to derive nuclear forces and current operators in harmony with the chiral symmetry of QCD. The expansion parameter is given by the ratio $Q / \Lambda$ where $Q$ is the soft scale associated with the pion mass and/or external nucleon momenta and $\Lambda$ is the pertinent hard scale. For a given connected irreducible diagram with $N$ nucleons, $L$ pion loops and $V_{i}$ vertices of type $i$, the power $\nu$ of the soft scale $Q$ which determines its importance can be obtained based on naive dimensional analysis (i.e. assuming clas-

\#2 An alternative framework based on the perturbative treatment of the pion exchange contributions has been introduced by Kaplan, Savage and Wise $(39 ; 40)$, see $(48 ; 87)$ for review articles. As shown in Refs. (132-134), the perturbative inclusion of pions does not allow to significantly increase the applicability range of the theory as compared to pionless EFT. For yet different proposals to include pions in EFT for the nucleon-nucleon system see Refs. (135-137). sical scaling dimensions for various operators in the effective Lagrangian):

$$
\nu=-4+2 N+2 L+\sum_{i} V_{i} \Delta_{i}, \quad \Delta_{i}=d_{i}+\frac{1}{2} n_{i}-2 .
$$

Here, $n_{i}$ is the number of nucleon field operators and $d_{i}$ the number of derivatives and/or insertions of $M_{\pi}$. The spontaneously broken chiral symmetry of QCD guarantees $\Delta_{i} \geq 0$. As a consequence, the chiral dimension $\nu$ is bounded from below, and only a finite number of diagrams contribute at a given order. In addition, Eq. (2.8) provides a natural explanation to the dominance of the two-nucleon interactions and the hierarchy of nuclear forces observed in nuclear physics. In particular, it implies that two-, three-, and four-nucleon forces start to contribute at orders $\nu=0,2$ and 4, respectively. Notice that as argued in Ref. (131), the nucleon mass $m$ should be counted as $Q / m \sim Q^{2} / \Lambda^{2}$ (which implies that $m \gg \Lambda$ ) in order to maintain consistency with the appearance of shallow-lying bound states. ${ }^{33}$ Notice further that according to this counting rule, the momentum scale associated with the real pion production is treated as the hard scale, $\sqrt{m M_{\pi}} \sim \Lambda$, and needs not be explicitly kept track of, see also (138) and section II.E for a related discussion. Clearly, such a framework is only applicable at energies well below the pion production threshold. We also emphasize that the validity of the naive dimensional scaling rules for few-nucleon contact operators has been questioned in Refs. $(139 ; 140)$. We will come back to this issue in section II.C.

Before discussing the chiral expansion of the nuclear forces it is important to clarify the relation between the underlying chiral Lagrangian for pions and nucleons and the nuclear Hamiltonian we are finally interested in. The derivation of the nuclear potentials from field theory is an old and extensively studied problem in nuclear physics. Different approaches have been developed in the fifties of the last century in the context of the so-called meson theory of nuclear forces, see e.g. the review article (141). In the modern framework of chiral EFT, the most frequently used methods besides the already mentioned time-ordered perturbation theory are the ones based on S-matrix and the unitary transformation. In the former scheme, the nuclear potential is defined through matching the amplitude to the iterated Lippmann-Schwinger equation (142). In the second approach, the potential is obtained by applying an appropriately choosen unitary transformation to the underlying pion-nucleon Hamiltonian which eliminates the coupling between the purely nucleonic Fock space states and the ones which contain pions, see (143) for more details. We stress that both methods lead to energy-independent interactions as opposed by the ones obtained in time-ordered perturbation

\footnotetext{
\#3 This statement only applies for the power counting based on
} naive dimensional analysis. 
theory. The energy independence of the potential is a welcome feature which enables applications to three- and more-nucleon systems.

We are now in the position to discuss the structure of the nuclear force at lowest orders of the chiral expansion. The leading-order ( $\mathrm{LO}$ ) contribution results, according to Eq. (2.8), from two-nucleon tree diagrams constructed from the Lagrangian of lowest dimension $\Delta_{i}=0, \mathcal{L}^{(0)}$, which has the following form in the heavy-baryon formulation $(144 ; 145)$ :

$$
\begin{aligned}
\mathcal{L}_{\pi}^{(0)} & =\frac{F^{2}}{4}\left\langle\nabla^{\mu} U \nabla_{\mu} U^{\dagger}+\chi_{+}\right\rangle \\
\mathcal{L}_{\pi N}^{(0)} & =\bar{N}\left(i v \cdot D+\stackrel{\circ}{g}_{A} u \cdot S\right) N \\
\mathcal{L}_{N N}^{(0)} & =-\frac{1}{2} C_{S}(\bar{N} N)(\bar{N} N)+2 C_{T}(\bar{N} S N) \cdot(\bar{N} S N)
\end{aligned}
$$

where $N, v_{\mu}$ and $S_{\mu} \equiv(1 / 2) i \gamma_{5} \sigma_{\mu \nu} v^{\nu}$ denote the large component of the nucleon field, the nucleon four-velocity and the covariant spin vector, respectively. The brackets $\langle\ldots\rangle$ denote traces in the flavor space while $F$ and $\stackrel{\circ}{g}_{A}$ refer to the chiral-limit values of the pion decay and the nucleon axial vector coupling constants. The low-energy constants (LECs) $C_{S}$ and $C_{T}$ determine the strength of the leading NN short-range interaction. Further, the unitary $2 \times 2$ matrix $U(\boldsymbol{\pi})=u^{2}(\boldsymbol{\pi})$ in the flavor space collects the pion fields,

$$
U(\boldsymbol{\pi})=1+\frac{i}{F} \boldsymbol{\tau} \cdot \boldsymbol{\pi}-\frac{1}{2 F^{2}} \boldsymbol{\pi}^{2}+\mathcal{O}\left(\pi^{3}\right),
$$

where $\tau_{i}$ denotes the isospin Pauli matrix. The covariant derivatives of the nucleon and pion fields are defined via $D_{\mu}=\partial_{\mu}+\left[u^{\dagger}, \partial_{\mu} u\right] / 2$ and $u_{\mu}=i\left(u^{\dagger} \partial_{\mu} u-u \partial_{\mu} u^{\dagger}\right)$. The quantity $\chi_{+}=u^{\dagger} \chi u^{\dagger}+u \chi^{\dagger} u$ with $\chi=2 B \mathcal{M}$ involves the explicit chiral symmetry breaking due to the finite light quark masses, $\mathcal{M}=\operatorname{diag}\left(m_{u}, m_{d}\right)$. The constant $B$ is related to the value of the scalar quark condensate in the chiral limit, $\langle 0|\bar{u} u| 0\rangle=-F^{2} B$, and relates the pion mass $M_{\pi}$ to the quark mass $m_{q}$ via $M_{\pi}^{2}=2 B m_{q}+\mathcal{O}\left(m_{q}^{2}\right)$. For more details on the notation and the complete expressions for the pion-nucleon Lagrangian including up to four derivatives $/ M_{\pi}$-insertions the reader is referred to (146). Expanding the effective Lagrangian in Eqs. (2.9) in powers of the pion fields one can easily verify that the only possible connected two-nucleon tree diagrams are the one-pion exchange and the contact one, see the first line in Fig. 12, yielding the following potential in the two-nucleon center-of-mass system (CMS):

$$
V_{2 \mathrm{~N}}^{(0)}=-\frac{g_{A}^{2}}{4 F_{\pi}^{2}} \frac{\vec{\sigma}_{1} \cdot \vec{q} \vec{\sigma}_{2} \cdot \vec{q}}{\vec{q}^{2}+M_{\pi}^{2}} \boldsymbol{\tau}_{1} \cdot \boldsymbol{\tau}_{2}+C_{S}+C_{T} \vec{\sigma}_{1} \cdot \vec{\sigma}_{2}
$$

where the superscript of $V_{2 \mathrm{~N}}$ denotes the chiral order $\nu$, $\sigma_{i}$ are the Pauli spin matrices, $\vec{q}=\vec{p}^{\prime}-\vec{p}$ is the nucleon momentum transfer and $\vec{p}\left(\vec{p}^{\prime}\right)$ refers to initial (final) nucleon momenta in the CMS. Further, $F_{\pi}=92.4 \mathrm{MeV}$ and $g_{A}=1.267$ denote the pion decay and the nucleon axial coupling constants, respectively.
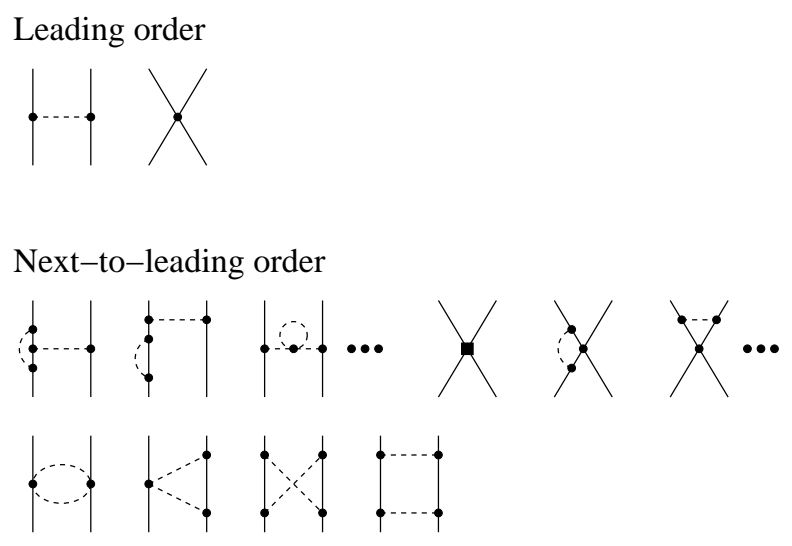

Next-to-next-to-leading order

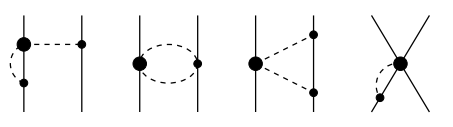

Next-to-next-to-next-to-leading order

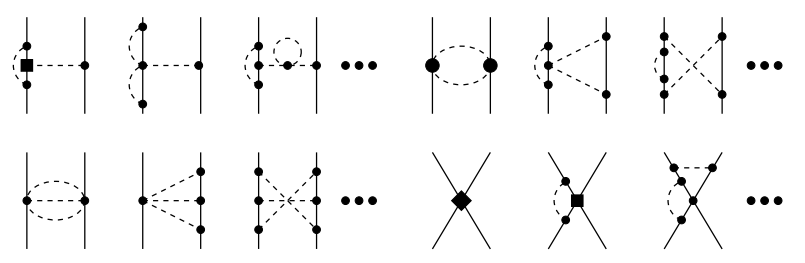

FIG. 12 Chiral expansion of the two-nucleon force up to $\mathrm{N}^{3} \mathrm{LO}$. Solid dots, filled circles, squares and diamonds denote vertices with $\Delta_{i}=0,1,2$ and 4 , respectively. Only irreducible contributions of the diagrams are taken in to account as explained in the text.

The first corrections to the LO result are suppressed by two powers of the low-momentum scale. The absence of the contributions at order $\nu=1$ can be traced back to parity conservation which forbids $(\bar{N} N)(\bar{N} N)$ vertices with one spatial derivative and $\pi N N$ vertices with two derivatives (i. e. $\Delta_{i}=1$ ). The next-to-leadingorder (NLO) contributions to the $2 \mathrm{NF}$ therefore result from tree diagrams with one insertion of the $\Delta_{i}=2$ interaction and one-loop diagrams constructed from the lowest-order vertices, see Fig. 12. The relevant terms in the effective Lagrangian read (147)

$$
\begin{aligned}
\mathcal{L}_{\pi}^{(2)} & =\frac{l_{3}}{16}\left\langle\chi_{+}\right\rangle^{2}+\frac{l_{4}}{16}\left(2\left\langle\nabla_{\mu} U \nabla^{\mu} U^{\dagger}\right\rangle\left\langle\chi_{+}\right\rangle\right. \\
& \left.+2\left\langle\chi^{\dagger} U \chi^{\dagger} U+\chi U^{\dagger} \chi U^{\dagger}\right\rangle-4\left\langle\chi^{\dagger} \chi\right\rangle-\left\langle\chi_{-}\right\rangle^{2}\right) \\
& +\ldots, \\
\mathcal{L}_{\pi N}^{(2)} & =\bar{N}\left(\frac{1}{2 \stackrel{\circ}{m}}(v \cdot D)^{2}-\frac{1}{2 \stackrel{\circ}{m}} D \cdot D+d_{16} S \cdot u\left\langle\chi_{+}\right\rangle\right. \\
& \left.+i d_{18} S^{\mu}\left[D_{\mu}, \chi_{-}\right]+\ldots\right) N,
\end{aligned}
$$




$$
\begin{aligned}
\mathcal{L}_{N N}^{(2)} & =-\tilde{C}_{1}((\bar{N} D N) \cdot(\bar{N} D N)+((D \bar{N}) N) \cdot((D \bar{N}) N)) \\
& -2\left(\tilde{C}_{1}+\tilde{C}_{2}\right)(\bar{N} D N) \cdot((D \bar{N}) N) \\
& -\tilde{C}_{2}(\bar{N} N) \cdot\left(\left(D^{2} \bar{N}\right) N+\bar{N} D^{2} N\right)+\ldots
\end{aligned}
$$

where $l_{i}, d_{i}$ and $\tilde{C}_{i}$ denote further LECs and $\stackrel{\circ}{m}$ is the nucleon mass in the chiral limit. The ellipses in the pion and pion-nucleon Lagrangians refer to terms which do not contribute to the nuclear force at NLO. In the case of the nucleon-nucleon Lagrangian $\mathcal{L}_{N N}^{(2)}$ only a few terms are given explicitly. The complete reparametrizationinvariant set of terms can be found in (148). The NLO contributions to the two-nucleon potential have been first considered in $(149 ; 150)$ utilizing the framework of timeordered perturbation theory. The corresponding energyindependent expressions have been worked out in (151) using the method described in (152) and then re-derived in (142) using an S-matrix-based approach and, independently, in $(143 ; 153)$ based on the method of unitary transformation. The one-pion $(1 \pi)$ exchange diagrams at NLO do not produce any new momentum dependence. Apart from renormalization of various LECs in Eq. (2.11), one obtains the leading contribution to the Goldberger-Treiman discrepancy (154)

$$
\frac{g_{\pi N}}{m}=\frac{g_{A}}{F_{\pi}}-\frac{2 M_{\pi}^{2}}{F_{\pi}} d_{18}+\ldots
$$

where the ellipses refer to higher-order terms. Similarly, loop diagrams involving NN short-range interactions only lead to $\left(M_{\pi}\right.$-dependent) shifts in the LO contact terms. The remaining contributions to the $2 \mathrm{NF}$ due to higherorder contact interactions and two-pion exchange have the form:

$$
\begin{aligned}
V_{2 \mathrm{~N}}^{(2)} & =-\frac{\boldsymbol{\tau}_{1} \cdot \boldsymbol{\tau}_{2}}{384 \pi^{2} F_{\pi}^{4}} L^{\tilde{\Lambda}}(q)\left(4 M_{\pi}^{2}\left(5 g_{A}^{4}-4 g_{A}^{2}-1\right)\right. \\
& \left.+\vec{q}^{2}\left(23 g_{A}^{4}-10 g_{A}^{2}-1\right)+\frac{48 g_{A}^{4} M_{\pi}^{4}}{4 M_{\pi}^{2}+\vec{q}^{2}}\right) \\
& -\frac{3 g_{A}^{4}}{64 \pi^{2} F_{\pi}^{4}} L^{\tilde{\Lambda}}(q)\left(\vec{\sigma}_{1} \cdot \vec{q} \vec{\sigma}_{2} \cdot \vec{q}-\vec{\sigma}_{1} \cdot \vec{\sigma}_{2} \vec{q}^{2}\right) \\
& +C_{1} \vec{q}^{2}+C_{2} \vec{k}^{2}+\left(C_{3} \vec{q}^{2}+C_{4} \vec{k}^{2}\right) \vec{\sigma}_{1} \cdot \vec{\sigma}_{2} \\
& +i C_{5} \frac{1}{2}\left(\vec{\sigma}_{1}+\vec{\sigma}_{2}\right) \cdot \vec{q} \times \vec{k}+C_{6} \vec{q} \cdot \vec{\sigma}_{1} \vec{q} \cdot \vec{\sigma}_{2} \\
& +C_{7} \vec{k} \cdot \vec{\sigma}_{1} \vec{k} \cdot \vec{\sigma}_{2},
\end{aligned}
$$

where $q \equiv|\vec{q}|$ and the LECs $C_{i}$ can be written as linear combinations of $\tilde{C}_{i}$ in Eq. (2.12). The loop function $L^{\tilde{\Lambda}}(q)$ is defined in the spectral function regularization (SFR) $(155 ; 156)$ as

$$
L^{\tilde{\Lambda}}(q)=\theta\left(\tilde{\Lambda}-2 M_{\pi}\right) \frac{\omega}{2 q} \ln \frac{\tilde{\Lambda}^{2} \omega^{2}+q^{2} s^{2}+2 \tilde{\Lambda} q \omega s}{4 M_{\pi}^{2}\left(\tilde{\Lambda}^{2}+q^{2}\right)},
$$

where we have introduced the following abbreviations: $\omega=\sqrt{4 M_{\pi}^{2}+\vec{q}^{2}}$ and $s=\sqrt{\tilde{\Lambda}^{2}-4 M_{\pi}^{2}}$. Here, $\tilde{\Lambda}$ denotes
Next-to-leading order

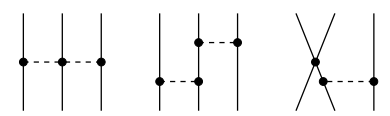

Next-to-next-to-leading order

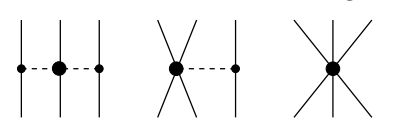

Next-to-next-to-next-to-leading order
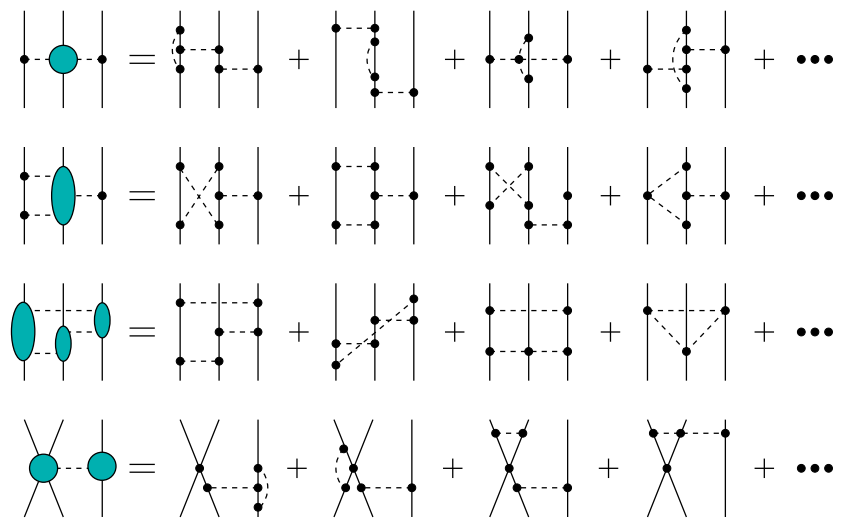

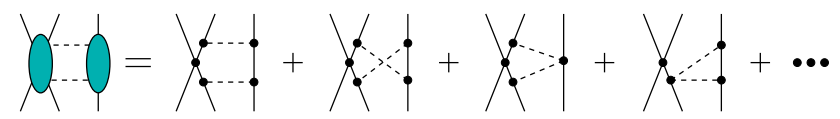

FIG. 13 Chiral expansion of the three-nucleon force up to $\mathrm{N}^{3}$ LO. Diagrams in the first line (NLO) yield vanishing contributions to the $3 \mathrm{NF}$ if one uses energy-independent formulations as explained in the text. The five topologies at $\mathrm{N}^{3} \mathrm{LO}$ involve the two-pion exchange, one-pion-two-pionexchange, ring, contact-one-pion exchange and contact-twopion-exchange diagrams in order. Shaded blobs represent the corresponding amplitudes. For remaining notation see Fig. 12.

the ultraviolet cutoff in the mass spectrum of the twopion-exchange potential. If dimensional regularization (DR) is employed, the expression for the loop function simplifies to

$$
L(q)=\lim _{\tilde{\Lambda} \rightarrow \infty} L^{\tilde{\Lambda}}(q)=\frac{\omega}{q} \ln \frac{\omega+q}{2 M_{\pi}} .
$$

In addition to the two-nucleon contributions, at NLO one also needs to consider three-nucleon diagrams shown in the first line of Fig. 13. The first diagram does not involve reducible topologies and, therefore, can be dealt with using the Feynman graph technique. It is then easy to verify that its contribution is shifted to higher orders due to the additional suppression by the factor of $1 / m$ caused by the appearance of time derivative at the leading-order $\pi \pi \bar{N} N$ vertex, the so-called WeinbergTomozawa vertex. The two remeining diagrams have 
been considered by Weinberg $(130 ; 131)$ and later by Ordonez and van Kolck (157) using the energy-dependent formulation based on time-ordered perturbation theory. In this approach, it was shown that the resulting $3 \mathrm{NF}$ cancels exactly (at the order one is working) against the recoil correction to the $2 \mathrm{NF}$ when the latter is iterated in the dynamical equation. In energy-independent approaches such as e.g. the method of unitary transformation which are employed in most of the existing fewnucleon calculations one observes that the irreducible contributions from the last two diagrams in the first line of Fig. 13 are suppressed by the factor $1 / \mathrm{m}$ and thus occur at higher orders $(148)$, see also $(158 ; 159)$. Consequently, there is no $3 \mathrm{NF}$ at NLO in the chiral expansion.

The contributions at next-to-next-to-leading order $\left(\mathrm{N}^{2} \mathrm{LO}\right)$ involve one-loop diagrams with one insertion of the subleading vertices of dimension $\Delta_{i}=1$, see Fig. 12 . The corresponding Lagrangians read:

$$
\begin{aligned}
\mathcal{L}_{\pi N}^{(1)} & =\bar{N}\left(c_{1}\left\langle\chi_{+}\right\rangle+c_{2}(v \cdot u)^{2}+c_{3} u \cdot u\right. \\
& \left.+c_{4}\left[S^{\mu}, S^{\nu}\right] u_{\mu} u_{\nu}+c_{5}\left\langle\hat{\chi}_{+}\right\rangle\right) N, \\
\mathcal{L}_{\pi N N}^{(1)} & =\frac{D}{2}(\bar{N} N)(\bar{N} S \cdot u N),
\end{aligned}
$$

where $\hat{\chi}_{+} \equiv \chi_{+}-\left\langle\chi_{+}\right\rangle / 2$ and $D, c_{i}$ are the LECs. The $1 \pi$-exchange loop diagram again only lead to renormalization of the corresponding LECs. Similarly, the contribution from the last diagram which involves the twonucleon contact interaction can be absorbed into a redefinition of the LECs $C_{S, T}$ and $C_{i}$ in Eqs. (2.11), (2.14) (provided one is not interested in the quark mass dependence of the nuclear force). Further, the football diagram yields vanishing contribution due to the antisymmetric (with respect to pion isospin quantum numbers) nature of the Weinberg-Tomozawa vertex. Thus, the only nonvanishing contribution at this order results from the $2 \pi$ exchange triangle diagram:

$$
\begin{aligned}
V_{2 \mathrm{~N}}^{(3)} & =-\frac{3 g_{A}^{2}}{16 \pi F_{\pi}^{4}}\left(2 M_{\pi}^{2}\left(2 c_{1}-c_{3}\right)-c_{3} \vec{q}^{2}\right)\left(2 M_{\pi}^{2}+\vec{q}^{2}\right) \\
& \times A^{\tilde{\Lambda}}(q)-\frac{g_{A}^{2} c_{4}}{32 \pi F_{\pi}^{4}} \boldsymbol{\tau}_{1} \cdot \boldsymbol{\tau}_{2}\left(4 M_{\pi}^{2}+q^{2}\right) A^{\tilde{\Lambda}}(q) \\
& \times\left(\vec{\sigma}_{1} \cdot \vec{q} \vec{\sigma}_{2} \cdot \vec{q}-\vec{q}^{2} \vec{\sigma}_{1} \cdot \vec{\sigma}_{2}\right)
\end{aligned}
$$

where the loop function $A^{\tilde{\Lambda}}(q)$ is given by

$$
A^{\tilde{\Lambda}}(q)=\theta\left(\tilde{\Lambda}-2 M_{\pi}\right) \frac{1}{2 q} \arctan \frac{q\left(\tilde{\Lambda}-2 M_{\pi}\right)}{q^{2}+2 \tilde{\Lambda} M_{\pi}} .
$$

In DR, the expression for $A(q)$ takes the following simple form:

$$
A(q) \equiv \lim _{\tilde{\Lambda} \rightarrow \infty} A^{\tilde{\Lambda}}(q)=\frac{1}{2 q} \arctan \frac{q}{2 M_{\pi}} .
$$

Notice that the triangle diagram also generates shortrange contributions which may be absorbed into redefinition of contact interactions. The isoscalar central contribution proportional to the LEC $c_{3}$ is attractive and very strong. It is by far the strongest two-pion exchange contribution and reaches a few $10 \mathrm{~s}$ of $\mathrm{MeV}$ (depending on the choice of regularization) at internucleon distances of the order $r \sim M_{\pi}^{-1}$. The origin of the unnaturally strong subleading $2 \pi$-exchange contributions can be traced back to the (numerically) large values of the LECs $c_{3,4}$ and is well understood in terms of resonance exchange related to $\Delta$ excitation (160). We will come back to this issue in section II.D where the chiral EFT formulation with explicit $\Delta$ degrees of freedom will be discussed. The central $2 \pi$-exchange potential was also calculated by Robilotta (161) using the infraredreglarized version of chiral EFT which enables to sum up a certain class of relativistic corrections (162). He found that the results in the heavy-baryon limit overestimate the ones obtained using infrared regularization by about $25 \%$, see also (163) for a related discussion. Last but not least, the chiral $2 \pi$-exchange potential up to $\mathrm{N}^{2} \mathrm{LO}$ has been tested in the Nijmegen partial wave analysis (PWA) of both proton-proton and neutron-proton data $(164 ; 165)$ where also an attempt has been done to determine the values of the LECs $c_{3,4}$. As demonstrated in these studies, the representation of the (strong) longrange interaction based on the combination of the $1 \pi$ and the chiral $2 \pi$-exchange potentials rather than on the pure $1 \pi$-exchnge potential allows to considerably reduce the number of phenomenological parameters entering the energy-dependent boundary conditions which are needed to parametrize the missing short- and medium-range interactions. Also the extracted values of the LECs $c_{3,4}$ agree reasonably well with various determinations in the pion-nucleon system. These studies provide a beautiful confirmation of the important role of the $2 \pi$-exchange potential in nucleon-nucleon scattering observables, see, however, Ref. (166) for a criticism. For a similar work utilizing the distorted-wave methods the reader is referred to $(167 ; 168)$.

The first nonvanishing contributions to the $3 \mathrm{NF}$ also show up at $\mathrm{N}^{2} \mathrm{LO}$ and arise from tree diagrams shown in Fig. 13 which involve a single insertion of the subleading vertices $\mathcal{L}^{(1)}$ in Eq. (2.17) and

$$
\mathcal{L}_{N N N}^{(1)}=-\frac{1}{2}(\bar{N} N)(\bar{N} \boldsymbol{\tau} N) \cdot(\bar{N} \boldsymbol{\tau} N) .
$$

where $E$ is a low-energy constant. The corresponding $3 \mathrm{NF}$ expression read:

$$
\begin{aligned}
V_{3 N}^{(3)}= & \frac{g_{A}^{2}}{8 F_{\pi}^{4}} \frac{\vec{\sigma}_{1} \cdot \vec{q}_{1} \vec{\sigma}_{3} \cdot \vec{q}_{3}}{\left[q_{1}^{2}+M_{\pi}^{2}\right]\left[q_{3}^{2}+M_{\pi}^{2}\right]}\left[\boldsymbol { \tau } _ { 1 } \cdot \boldsymbol { \tau } _ { 3 } \left(-4 c_{1} M_{\pi}^{2}\right.\right. \\
& \left.\left.+2 c_{3} \vec{q}_{1} \cdot \vec{q}_{3}\right)+c_{4} \boldsymbol{\tau}_{1} \times \boldsymbol{\tau}_{3} \cdot \boldsymbol{\tau}_{2} \vec{q}_{1} \times \vec{q}_{3} \cdot \vec{\sigma}_{2}\right] \\
- & \frac{g_{A} D}{8 F_{\pi}^{2}} \frac{\vec{\sigma}_{3} \cdot \vec{q}_{3}}{q_{3}^{2}+M_{\pi}^{2}} \boldsymbol{\tau}_{1} \cdot \boldsymbol{\tau}_{3} \vec{\sigma}_{1} \cdot \vec{q}_{3} \\
+ & \frac{1}{2} E \boldsymbol{\tau}_{2} \cdot \boldsymbol{\tau}_{3},
\end{aligned}
$$

where the subscripts refer to the nucleon labels and $\vec{q}_{i}=\vec{p}_{i}{ }^{\prime}-\vec{p}_{i}$, with $\vec{p}_{i}{ }^{\prime}$ and $\vec{p}_{i}$ being the final and initial 
momenta of the nucleon $i$. The expressions in Eq. (2.22) correspond to a particular choice of nucleon labels. The full expression for the $3 \mathrm{NF}$ results by taking into account all possible permutations of the nucleons (for three nucleons there are altogether six permutations), i.e.:

$$
V_{3 \mathrm{~N}}^{\mathrm{full}}=V_{3 \mathrm{~N}}+\text { all permutations . }
$$

We further emphasize that the expressions for the $3 \mathrm{NF}$ given in Refs. $(157 ; 169)$ contain one redundant $1 \pi$ exchange and two redundant contact interactions. As shown in (123), only one independent linear combination contributes in each case if one considers matrix elements between antisymmetrized few-nucleon states, see also (92) for a related discussion.

We now turn to next-to-next-to-next-to-leading order $\left(\mathrm{N}^{3} \mathrm{LO}\right)$ and discuss first the corrections to the 2NF. As follows from Eq. (2.8), one has to account for contributions from tree diagrams with one insertion from $\mathcal{L}^{(4)}$ or two insertions from $\mathcal{L}^{(2)}$, one-loop diagrams with one insertion from $\mathcal{L}^{(2)}$ or two insertions from $\mathcal{L}^{(1)}$ as well as two-loop graphs constructed from the lowest-order vertices, see Fig. 12. Apart from renormalization of various LECs, the $1 \pi$-exchange potential receives at this order (in the scheme based on the counting $\left.m \sim \mathcal{O}\left(\Lambda^{2} / M_{\pi}\right)\right)$ the first relativistic corrections proportional to $\mathrm{m}^{-2}$. These are scheme-dependent and have to be chosen consistently with the $1 / m$-corrections to the $2 \pi$-exchange potential and the relativistic extension of the dynamical equation, see (170) for a comprehensive discussion. The two-pion exchange contributions at $\mathrm{N}^{3} \mathrm{LO}$ were worked out by Kaiser (171) based on the one-loop representation of the $\pi N$ scattering amplitude. We refrain from giving here the rather involved expressions for the sub-subleading $2 \pi$ exchange potential and refer to the original work (171) where the results are given in terms of the corresponding spectral functions. For certain classes of contributions, the integrals over the two-pion exchange spectrum could be performed analytically and are given in Ref. (172). Notice further that the subleading (i.e. the ones proportional to $\mathrm{m}^{-2}$ ) relativistic corrections of the $2 \pi$-exchange range have also been worked out by Kaiser (173). In the counting scheme with $m \sim \mathcal{O}\left(\Lambda^{2} / M_{\pi}\right)$, these terms, however, would only appear at $\mathrm{N}^{5} \mathrm{LO}$. It should also be emphasized that the $\mathrm{N}^{3} \mathrm{LO}$ contributions to the $2 \pi$-exchange potential were worked out in the covariant version of chiral EFT (more precisely, using the formulation by Becher and Leutwyler (162)) by Higa et al. (174-176).

$3 \pi$-exchange contributions also appear at this order in the chiral expansion and have been worked out in Refs. $(177 ; 178)$, see also Ref. (179) for a related work. The resulting potentials turn out to be rather weak. For example, the strongest contribution is of the isoscalar spin-spin type (i.e. proportional to $\vec{\sigma}_{1} \cdot \vec{\sigma}_{2}$ ) and about 10 times weaker than the corresponding $2 \pi$-exchange contribution at the same order at relative distances $r \sim M_{\pi}^{-1}$. It should, however, be emphasized that the subleading $3 \pi$-exchange contributions at $\mathrm{N}^{4} \mathrm{LO}$ are larger in size (180) which, again, can be traced back to the large val- ues of the LECs $c_{i}$. Finally, the last type of the $2 \mathrm{NF}$ corrections at this order results from diagrams involving contact interactions. The most general polynomial (in momenta) representation of the short-range part of the potential involves, apart from the two leading and seven subleading terms given in Eqs. (2.11) and (2.14) fifteen new contact interactions (in the isospin invariant sector) yielding in total 24 LECs to be determined from nucleonnucleon data.

The $3 \mathrm{NF}$ contributions at $\mathrm{N}^{3} \mathrm{LO}$ feed into five different topologies, see Fig. 13, and are currently being worked out. Currently, the expressions for the first three topologies which do not involve short-range contact interactions are available. The one-loop corrections to the $2 \pi$ exchange diagrams can, to a large extent, be accounted for by a finite shift $c_{i} \rightarrow \bar{c}_{i}=c_{i}+\delta c_{i}$ of the LECs $c_{i}$ $(181 ; 182)$

$$
\delta c_{1}=-\frac{g_{A}^{2} M_{\pi}}{64 \pi F_{\pi}^{2}}, \quad \delta c_{3}=-\delta c_{4}=\frac{g_{A}^{4} M_{\pi}}{16 \pi F_{\pi}^{2}} .
$$

Numerically, these corrections are of the order of $20 \%$ of the corresponding LECs and are consistent with the difference in values of $c_{i}$ between the order- $Q^{2}$ and $Q^{3}$ determinations from the pion-nucleon system, see $(160 ; 183-$ $185)$. The only $2 \pi$-exchange contribution that cannot be cast into redefinition of the LECs $c_{i}$ arises from the diagram which involves pions interacting in flight, see (181; 182) for the explicit expression. We also emphasize that there are no $2 \pi$-exchange contributions from tree diagrams with one insertion from $\mathcal{L}_{\pi N}^{(2)}$ in Eq. (2.12) (except for the relativistic corrections). This is because diagrams involving subleading $\pi N N$ interaction do not yield any irreducible contributions while the ones with the $\pi \pi N N$-vertices of dimension $\nu=2$ involve at least one time derivative and are, therefore, suppressed by a factor of $1 / \mathrm{m}$. This observation is consistent with the absence of logarithmic ultraviolet divergences in the loop diagrams. In this context, it should be emphasized that the requirement of renormalizability at $\mathrm{N}^{3} \mathrm{LO}$ (and, presumably, also at higher orders) was found to impose strong constraints on the unitary ambiguity in the form of the resulting nuclear potentials. This issue is discussed extensively in (186) and may remind one of the recent findings in the context of large- $N_{c}$ QCD (187-189) where it was shown that the multiple-meson-exchange potential derived in the energy-dependent formulation is inconsistent with large- $N_{C}$ counting rules. The consistency could be maintained using a different (but equivalent up to the considered order) form of the potential based on the energy-independent formalism, see Ref. (189) for more details. The contributions from the two-pion-onepion exchange and ring diagrams are given explicitly in Ref. (181) where expressions are shown both in momentum and coordinate spaces. Especially in the case of ring diagrams where loop integrals involve two independent external momenta and, therefore, yield rather involved expressions in momentum space. It is advantageous to switch to coordinate space where a much more compact 

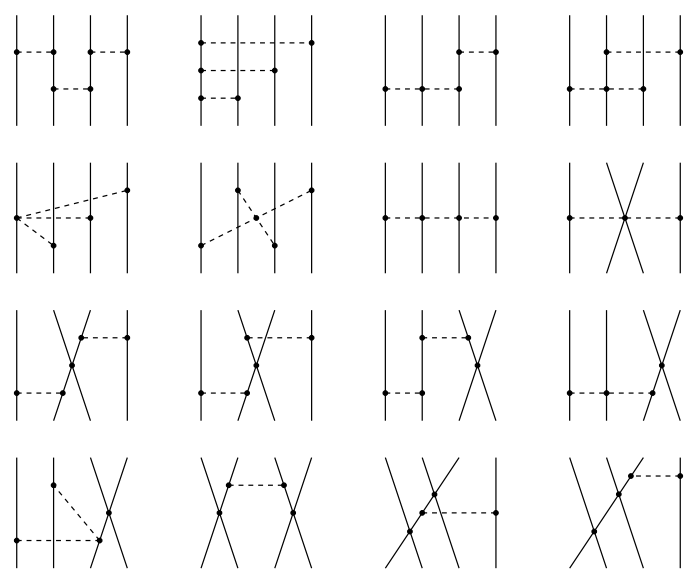

FIG. 14 Diagrams contributing to the four-nucleon force at $\mathrm{N}^{3} \mathrm{LO}$. For notation see Fig. 12 .

representation emerges. Notice further that ring diagrams were already studied in the pioneering work (190). The calculation of the last two topologies involving the leading contact interactions is in progress. Last but not least, one should also take into account the leading relativistic $1 / m$-corrections to the NLO three-nucleon diagrams, see the first line in Fig. 13. Again, these contributions are scheme-dependent and should be chosen consistently with the relativistic corrections to the $2 \mathrm{NF}$ and the form of the dynamical equation. The $1 / m$-corrections to the $2 \pi$-exchange $3 \mathrm{NF}$ have already been worked out long time ago by Coon and Friar and are given in the most general form in Ref. (158). Notice further that at this order, one needs to account for the dependence of the $2 \mathrm{NF}$ on the total momentum of the $2 \mathrm{~N}$ system (effects due to drift of the CMS of a two-body subsystem). Such boosted $2 \mathrm{~N}$ operators may, in fact, also be viewed as $3 \mathrm{~N}$ operators. In the context of chiral EFT, this kind of corrections is discussed in (191).

The last type of $\mathrm{N}^{3} \mathrm{LO}$ contributions arises from fournucleon tree diagrams constructed from the lowest-order vertices, see in Fig. 14, which have been evaluated recently using the method of unitary transformation (186; 192). Notice that the first two diagrams in the second line were already discussed long ago, see e.g. (193; 194). Furthermore, it should be emphasized that disconnected diagrams calculated e.g. in (169) using time-ordered perturbation theory do not contribute to the nuclear force in the method of unitary transformation. It has been conjectured in Ref. (194) that $4 \mathrm{~N}$ diagrams which involve reducible topologies do not generate irreducible pieces in the amplitude and thus lead to vanishing 4NFs. While this is indeed the case for the leading $3 \mathrm{~N}$ diagrams at NLO, it is explicitly shown in Ref. $(186 ; 192)$ that many of the reducible-like diagrams in Fig. 14 do generate nonvanishing 4 NFs which are not suppressed by inverse powers of the nucleon mass. As a representative example, we give here the $g_{A}^{6}$-contribution which results entirely from the first diagram in Fig. 14 (the second graph appears to be truly reducible and does not produce any contribution to the $4 \mathrm{NF})$ :

$$
\begin{aligned}
V_{4 N}^{(4)}= & -\frac{2 g_{A}^{6}}{\left(2 F_{\pi}\right)^{6}} \frac{\vec{\sigma}_{1} \cdot \vec{q}_{1} \vec{\sigma}_{4} \cdot \vec{q}_{4}}{\left[\vec{q}_{1}^{2}+M_{\pi}^{2}\right]\left[\vec{q}_{12}^{2}+M_{\pi}^{2}\right]^{2}\left[\vec{q}_{4}^{2}+M_{\pi}^{2}\right]} \\
\times & {\left[\left(\boldsymbol{\tau}_{1} \cdot \boldsymbol{\tau}_{4} \boldsymbol{\tau}_{2} \cdot \boldsymbol{\tau}_{3}-\boldsymbol{\tau}_{1} \cdot \boldsymbol{\tau}_{3} \boldsymbol{\tau}_{2} \cdot \boldsymbol{\tau}_{4}\right) \vec{q}_{1} \cdot \vec{q}_{12} \vec{q}_{4} \cdot \vec{q}_{12}\right.} \\
& +\boldsymbol{\tau}_{1} \times \boldsymbol{\tau}_{2} \cdot \boldsymbol{\tau}_{4} \vec{q}_{1} \cdot \vec{q}_{12} \vec{q}_{12} \times \vec{q}_{4} \cdot \vec{\sigma}_{3} \\
& +\boldsymbol{\tau}_{1} \times \boldsymbol{\tau}_{3} \cdot \boldsymbol{\tau}_{4} \vec{q}_{4} \cdot \vec{q}_{12} \vec{q}_{1} \times \vec{q}_{12} \cdot \vec{\sigma}_{2} \\
& \left.+\boldsymbol{\tau}_{1} \cdot \boldsymbol{\tau}_{4} \vec{q}_{12} \times \vec{q}_{1} \cdot \vec{\sigma}_{2} \vec{q}_{12} \times \vec{q}_{4} \cdot \vec{\sigma}_{3}\right]+ \text { all perm., }
\end{aligned}
$$

where $\vec{q}_{12}=\vec{q}_{1}+\vec{q}_{2}=-\vec{q}_{3}-\vec{q}_{4}=-\vec{q}_{34}$ is the momentum transfer between the nucleon pairs 12 and 34 . The complete expression for the leading $4 \mathrm{NF}$ both in momentum and coordinate space can be found in Ref. (186). A rough estimation of the $4 \mathrm{NF}$ contributions to e.g. the $\alpha$-particle binding energy is provided by the strength of the corresponding $r$-space potentials expressed in terms of dimensionless variables $r_{i j} M_{\pi}$. One then finds e.g. for the $g_{A}^{6}$-terms $g_{A}^{6} M_{\pi}^{7}\left(16 \pi F_{\pi}^{2}\right)^{-3} \sim 50 \mathrm{keV}$. This agrees qualitatively with a more accurate numerical estimation carried out in Ref. (195) which, however, still involved severe approximations to simplify the calculations.

So far we only discussed isospin-invariant contributions to the nuclear forces. It is well established that nuclear forces are charge dependent (for reviews see e.g. $(196 ; 197))$. For example, in the nucleon-nucleon ${ }^{1} S_{0}$ channel one has for the scattering lengths $a$ and the effective ranges $r$ (after removing electromagnetic effects)

$$
\begin{aligned}
a_{\mathrm{CIB}} & =\frac{1}{2}\left(a_{n n}+a_{p p}\right)-a_{n p}=5.64 \pm 0.40 \mathrm{fm} \\
r_{\mathrm{CIB}} & =\frac{1}{2}\left(r_{n n}+r_{p p}\right)-r_{n p}=0.03 \pm 0.06 \mathrm{fm}(2.2
\end{aligned}
$$

These numbers for charge independence breaking (CIB) are taken from the recent compilation of Machleidt (18). The charge independence breaking in the scattering lengths is large, of the order of $25 \%$, since $a_{n p}=$ $(-23.740 \pm 0.020) \mathrm{fm}$. Of course, it is magnified at threshold due to kinematic factors (as witnessed by the disappearance of the effect in the effective range). In addition, there are charge symmetry breaking (CSB) effects leading to different values for the $p p$ and $n n$ phase shifts/threshold parameters,

$$
\begin{aligned}
& a_{\mathrm{CSB}}=a_{p p}-a_{n n}=1.6 \pm 0.6 \mathrm{fm}, \\
& r_{\mathrm{CSB}}=r_{p p}-r_{n n}=0.10 \pm 0.12 \mathrm{fm} .
\end{aligned}
$$

Combining these numbers gives as central values $a_{n n}=$ $-18.9 \mathrm{fm}$ and $a_{p p}=-17.3 \mathrm{fm}$. Notice that this value for $a_{n n}$ is in agreement with the recent experimental determinations from the reaction $\pi^{-} d \rightarrow n n \gamma, a_{n n}=$ $-18.5 \pm 0.5 \mathrm{fm}$ (198), and the kinematically complete deuteron breakup reaction $n d \rightarrow n n p$ at $E_{\text {lab }}=13 \mathrm{MeV}$, $a_{n n}=-18.7 \pm 0.6 \mathrm{fm}$ (199). However, another recent experiment also based on the deuteron breakup reaction 
at $E_{\text {lab }}=25.2 \mathrm{MeV}$ yielded a considerably smaller value, $a_{n n}=-16.3 \pm 0.4 \mathrm{fm}(200)$. For a review of indirect methods to measure the ${ }^{1} S_{0} n n$ scattering length and the current experimental status for this observable see (201).

Within the Standard Model, isospin violation has its origin in the different masses of the up and down quarks and the electromagnetic interactions. Chiral EFT is well suited to explore the consequences of these two effects for low-energy dynamics of few- and many-nucleon systems. Consider first the strong isospin-violating effects. The QCD quark mass term can be written in the two-flavor case as

$$
\mathcal{L}_{\mathrm{mass}}^{\mathrm{QCD}}=-\frac{1}{2} \bar{q}\left(m_{u}+m_{d}\right)\left(1+\epsilon \tau^{3}\right) q,
$$

where the superscript of the Pauli isospin matrix denotes the corresponding Cartesian component and

$$
\epsilon \equiv \frac{m_{u}-m_{d}}{m_{u}+m_{d}} \sim-\frac{1}{3} .
$$

Here, the numerical estimation corresponds to the modified $\overline{\mathrm{MS}}$ subtraction scheme at a renormalization scale of $1 \mathrm{GeV}$ (202). In Eq. (2.28), the isoscalar term breaks chiral but preserves isospin symmetry. It is responsible e.g. for the nonvanishing pion mass $M^{2}=\left(m_{u}+m_{d}\right) B$, $M_{\pi}^{2}=M^{2}+\mathcal{O}\left(m_{u, d}^{2}\right)$, and generates a string of chiralsymmetry-breaking terms in the effective hadronic Lagrangian proportional to $M^{2 n}$ with $n=1,2, \ldots$. The isovector term gives rise to the strong isospin breaking and leads to hadronic effective interactions $\propto\left(\epsilon M^{2}\right)^{n}$. Consequently, the typical size of the strong isospin violation in hadronic observables is given by $\epsilon M_{\pi}^{2} / \Lambda^{2} \sim 1 \%$ if one takes $\Lambda=M_{\rho}$ (this, however, does not apply e.g. to the pion masses). The leading and subleading strong isospin-violating contributions are already incorporated in the Lagrangians $\mathcal{L}_{\pi}^{(2)}$ and $\mathcal{L}_{\pi N}^{(1,2)}$ in Eqs. (2.9), (2.12), (2.17) and correspond to terms involving $\chi, \chi_{ \pm}$. Notice that the strong isospin violation is additionally suppressed in the meson sector (due to G-parity). In particular, the charged-to-neutral pion mass difference is almost entirely of electromagnetic origin. Electromagnetic terms in the effective Lagrangian resulting from exchange of hard virtual photons can be generated using the method of external sources (35). All such terms are proportional to positive powers of the nucleon charge matrix $Q=e\left(1+\tau^{3}\right) / 2$ where $e$ denotes the electric charge. In addition, soft photons have to be included explicitly. For more details on the inclusion of virtual photons in chiral EFT the reader is referred to (203-209).

To explore isospin-breaking (IB) effects in nuclear forces/few-nucleon observables it is useful to relate the corresponding small parameters $\epsilon$ and $e$ to the chiral expansion parameter $M_{\pi} / \Lambda$. Clearly, this can be done in various ways. For example, in Refs. (210-212) the following rules have been adopted:

$$
\epsilon \sim e \sim \frac{M_{\pi}}{\Lambda} ; \quad \frac{e^{2}}{(4 \pi)^{2}} \sim \frac{M_{\pi}^{4}}{\Lambda^{4}} .
$$

Similar counting rules were also used in Refs. (213-218). Notice, however, that in the meson and single-baryon sectors one usually counts $\epsilon \sim 1$ but $e \sim M_{\pi} / \Lambda$. Utilizing the counting rules in Eq. (2.30), the leading and subleading IB contributions from the hard virtual photons have the form (208):

$$
\begin{aligned}
\mathcal{L}_{\pi, \mathrm{em}}^{(2)} & =C\left\langle Q U Q U^{\dagger}\right\rangle, \\
\mathcal{L}_{\pi N, \mathrm{em}}^{(3)} & =F^{2} \bar{N}\left(f_{1}\left\langle\tilde{Q}_{+}^{2}-\tilde{Q}_{-}^{2}\right\rangle+f_{2}\left\langle Q_{+}\right\rangle \tilde{Q}_{+}\right. \\
& \left.+f_{3}\left\langle\tilde{Q}_{+}^{2}+\tilde{Q}_{-}^{2}\right\rangle\right) N,
\end{aligned}
$$

where $\tilde{Q}_{ \pm} \equiv Q_{ \pm}-1 / 2\left\langle Q_{ \pm}\right\rangle$and $f_{i}$ refer to the corresponding LECs. The leading Lagrangians for the strong and electromagnetic IB contact interactions $\mathcal{L}_{N N \text {, str }}^{(3)}$ and $\mathcal{L}_{N N \text {, em }}^{(4)}$ are given explicitly in Ref. (210).

Let us first discuss IB contributions to the pion and nucleon masses. As already pointed out, the leading contribution to the charged-to-neutral pion mass difference is entirely of electromagnetic origin,

$$
\delta M_{\pi}^{2} \equiv M_{\pi^{ \pm}}^{2}-M_{\pi^{0}}^{2} \simeq \frac{2}{F^{2}} e^{2} C .
$$

The experimentally known pion mass difference $M_{\pi^{ \pm}}-$ $M_{\pi^{0}}=4.6 \mathrm{MeV}$ allows to fix the value of the $\operatorname{LEC} C$, $C=5.9 \cdot 10^{-5} \mathrm{GeV}^{4}$. Notice that the natural scale for this LEC is $F_{\pi}^{2} \Lambda^{2} /(4 \pi)^{2} \sim 3 \cdot 10^{-5} \mathrm{GeV}^{4}$ if one adopts $\Lambda \sim M_{\rho}$. Writing the nucleon mass $m$ as

$$
m \equiv \operatorname{diag}\left(m_{p}, m_{n}\right)=m+\frac{1}{2} \delta m \tau^{3},
$$

one obtains for the proton-to-neutron mass difference $\delta m$ :

$$
\delta m=-4 c_{5} \epsilon M_{\pi}^{2}-f_{2} e^{2} F_{\pi}^{2}+\ldots,
$$

where the ellipses refer to higher-order corrections. Notice that the $f_{3}$-term in Eq. (2.31) is isospin-invariant. On the other hand, the $f_{1}$-term does produce IB vertices with two and more pions but does not contribute to the leading electromagnetic nucleon mass shift. The LECs $c_{5}$ and $f_{2}$ can be determined from the strong and electromagnetic nucleon mass shifts:

$$
\begin{aligned}
& \left(m_{p}-m_{n}\right)^{\mathrm{str}}=(\delta m)^{\mathrm{str}}=-2.05 \pm 0.3 \mathrm{MeV} \\
& \left(m_{p}-m_{n}\right)^{\mathrm{em}}=(\delta m)^{\mathrm{em}}=0.76 \pm 0.3 \mathrm{MeV},
\end{aligned}
$$

which leads to $(207)$

$$
c_{5}=-0.09 \pm 0.01 \mathrm{GeV}^{-1}, \quad f_{2}=-0.45 \pm 0.19 \mathrm{GeV}^{-1} \text {. }
$$

The values for the strong and electromagnetic nucleon mass shifts are taken from (219). Notice that the recent lattice QCD result (220) for the strong nucleon mass shift $(\delta m)^{\mathrm{str}}=-2.26 \pm 0.57 \pm 0.42 \pm 0.10 \mathrm{MeV}$ is in agreement with the one of Ref. (219). We further emphasize that according to the counting rules in Eq. (2.30), the electromagnetic contribution to the nucleon mass shift is formally of higher order than the strong one. Based on 
naive dimensional analysis, these contributions are expected to be of the size $\left|(\delta m)^{\mathrm{str}}\right| \sim \epsilon M_{\pi}^{2} / M_{\rho} \sim 8 \mathrm{MeV}$ and $\left|(\delta m)^{\mathrm{em}}\right| \sim e^{2} M_{\rho} /(4 \pi)^{2} \sim 0.5 \mathrm{MeV}$.

We are now in the position to overview the structure of the IB nuclear forces. The general isospin structure of the two-nucleon force feeds, according to the classification of Henley and Miller (221), into the four classes: $V_{2 N}^{I}=$ $\alpha+\beta \boldsymbol{\tau}_{1} \cdot \boldsymbol{\tau}_{2}$ (isospin-invariant), $V_{2 N}^{I I}=\alpha \tau_{1}^{3} \tau_{2}^{3}$ (chargeindependence-breaking), $V_{2 N}^{I I I}=\alpha\left(\tau_{1}^{3}+\tau_{2}^{3}\right)$ (chargesymmetry-breaking) and $V_{2 N}^{I N}=\alpha\left(\tau_{1}^{3}-\tau_{2}^{3}\right)+\beta\left[\boldsymbol{\tau}_{1} \times \boldsymbol{\tau}_{2}\right]^{3}$ (issospin-mixing). Here, $\alpha$ and $\beta$ denote the corresponding space and spin operators. Notice that for the class-IV terms, $\beta$ has to be odd under a time reversal transformation. The most general isospin structure of the $3 \mathrm{NF}$ is worked out in Ref. (211). While the distinction between the class-I, II and III forces based on the conservation of the total isospin operator $\boldsymbol{T}=\left(\sum_{i} \boldsymbol{\tau}_{i}\right) / 2$ and chargesymmetry operator $P_{c s}=\exp \left(i \pi T_{2}\right)$ can be straightforwardly generalized to any number of nucleons, the conservation of the operator $\boldsymbol{T}^{2}$ responsible for the distinction between the class-III and IV 2NFs depends, in general, on the number of nucleons. In particular, the class-II and III $2 \mathrm{NFs}$ commute with the operator $\boldsymbol{T}_{2 N}^{2}$ (i.e. do not mix isospin in the $2 \mathrm{~N}$ system) but do not commute with $\boldsymbol{T}_{>2 N}^{2}$. For this reason, the general isospin structure of the $3 \mathrm{NF}$ was classified in Ref. (211) in terms of class-I, II and III contributions.

The dominant IB contribution to the $2 \mathrm{NF}$ occurs at NL $\varnothing$ (the slash indicates that we now use the power counting rules extended as in Eq. (2.30)) due to the charge-to-neutral pion mass difference in the $1 \pi$-exchange potential. It can be accounted for by taking the proper pion masses in the $1 \pi$-exchange potential for various physical channels:

$$
\begin{aligned}
V_{1 \pi}^{p p} & =V_{1 \pi}^{n n}=V_{1 \pi}\left(M_{\pi^{0}}\right), \\
V_{1 \pi}^{n p} & =-V_{1 \pi}\left(M_{\pi^{0}}\right)+2(-1)^{I+1} V_{1 \pi}\left(M_{\pi^{ \pm}}\right),
\end{aligned}
$$

where $I$ denotes the total isospin of the two-nucleon system and

$$
V_{1 \pi}\left(M_{\pi^{ \pm}}\right)=-\frac{g_{A}^{2}}{4 F_{\pi}^{2}} \frac{\vec{\sigma}_{1} \cdot \vec{q} \vec{\sigma}_{2} \cdot \vec{q}}{\vec{q}^{2}+M_{\pi}^{2}} .
$$

Notice that the resulting IB interaction conserves charge symmetry (i.e. class-II) and reaches about $\delta M_{\pi}^{2} / M_{\pi}^{2} \sim$ $7 \%$ of the strength of the isospin-invariant $1 \pi$-exchange potential. It is known to yield a sizeable contribution to the CIB in the ${ }^{1} S_{0} \mathrm{NN}$ scattering length, see e.g. (210). Another IB effect at the same order comes from the Coulomb interaction between the protons (class-II and III). We emphasize that effects of the purely electromagnetic interactions in two-nucleon scattering observables get enhanced under certain kinematical conditions (low energies and/or forward angles) due to the long-range nature of these interactions. Clearly, such an enhancement goes beyond the simple power counting rules in Eq. (2.30). Consequently, despite the fact that the first corrections to the point-like static one-photon exchange
(Coulomb interaction) due to recoil and two-photon exchange (222), pion loop contributions to the nucleon form factors $(223)$, vacuum polarization $(224 ; 225)$ and magnetic moment interaction (226) are suppressed by the factor $1 / \mathrm{m}^{2}$ and, according to the power counting, contribute at rather high orders, sizeable effects may show up in certain observables. For example, the magnetic moment interaction strongly affects the nucleon analyzing power $A_{y}$ at low energy and forward angles. Effects of subleading electromagnetic interactions were also investigated in $3 \mathrm{~N}$ continuum using phenomenological nuclear forces $(227 ; 228)$, see $(229)$ for a formulation based on pionless EFT.

The corrections to the IB $2 \mathrm{NF}$ at $\mathrm{N}^{2} \mathrm{~L} \emptyset$ are CSB and arise from charge dependence of the pion-nucleon coupling constant in the $1 \pi$-exchange potential and the derivative-less NN contact interaction $\propto m_{u}-m_{d}$ $(212 ; 214)$. Notice, however, that the energy-dependent Nijmegen PWA does not yield any evidence for charge dependence of the pion-nucleon coupling constant (230). The leading CIB contact interactions are of electromagnetic origin and (formally) start to contribute at $\mathrm{N}^{3} \mathrm{~L} \phi$. At this order, one also has to take into account further IB contributions to the $1 \pi$-exchange potential at the one-loop level which, to a large extent, can be accounted for by a further (charge-dependent) renormalization of the $\pi N$ coupling constants in Eq. (2.36). The only contributions which have a different momentum dependence and, therefore, cannot be cast into the form of Eq. (2.36) are the ones $\propto\left(\delta M_{\pi}\right)^{2}$ and the protonto-neutron mass difference which involve class-IV operators $(212 ; 217)$, see $(231)$ for a much earlier derivation of these terms. Notice that the power counting rules in Eq. (2.30) suggests the following hierarchy of the $2 \mathrm{NF}$ (213): $V_{2 N}^{I}>V_{2 N}^{I I}>V_{2 N}^{I I I}>V_{2 N}^{I V}$ which is consistent with the observations. Next, $\pi \gamma$-exchange also contributes at this order. The resulting CIB potential has been worked out by van Kolck at al. (232) and re-derived recently by Kaiser (223). It can be written in a rather compact way and leads to negligibly small effects in NN scattering. Kaiser also calculated subleading contributions to the $\pi \gamma$-exchange potential proportional to the large isovector magnetic moment $\kappa_{v}=4.7$ of the nucleon and found that the resulting potentials, which are also CIB, have a similar strength as the leading-order one (223). ${ }^{\# 4}$ IB $2 \pi$-exchange also starts to contribute at $\mathrm{N}^{3} \mathrm{~L} \emptyset$ and is driven by the neutral-to-charged pion mass difference (CIB) (215) and the strong contribution to the nucleon mass shift (CSB) $(212 ; 216 ; 233 ; 234)$, see (210) for the application to NN phase shifts. Finally, there are also the first IB 3NFs. While the dominant CIB $2 \pi$ exchange $2 \mathrm{NF}$ is generated by the pion mass difference,

\footnotetext{
\#4 Notice, however, that these corrections are suppressed by the factor $1 / m$ relative to the leading-order contributions and, therefore, appear formally at $\mathrm{N}^{5} \mathrm{~L} \phi$.
} 
the $3 \mathrm{~N}$ diagrams with one insertion of $\delta M_{\pi}^{2}$ at $\mathrm{N}^{3} \mathrm{~L} \emptyset$ are additionaly suppressed by the factor $1 / m$ (if one uses an energy-independent formulation), see the discussion about the 3NFs at NLO earlier in the text. The nonvanishing $3 \mathrm{NF}$ s at $\mathrm{N}^{3} \mathrm{~L} \emptyset$ result from $1 \pi$ - and $2 \pi$-exchange $3 \mathrm{~N}$ diagrams constructed with the leading-order isospininvariant vertices and a single insertion of $\delta m$ as well as $2 \pi$-exchange diagram with the leading IB $\pi \pi N N$ interactions $\propto f_{1,2}(211 ; 218)$. One finds that all these contributions are CSB except the one which is proportional to LEC $f_{1}$ and is CIB. We further emphasize that while the value of the LEC $f_{2}$ is determined by the electromagnetic nucleon mass shift, the value of the LEC $f_{1}$ is unknown. However, see (209) for an estimation of $f_{1}$ based on dimensional analysis and (235) for an attempt to determine $f_{1}$ from data.

Remarkably, even the $\mathrm{N}^{4} \mathrm{~L} \emptyset$ contributions to the twoand three-nucleon forces have been worked out. At this order, no new structures appear in the $1 \pi$-exchange potentials. The corrections to the leading IB $2 \pi$-exchange potential result from a single insertion of either the subleading isospin-conserving $\pi \pi N N$ vertices proportional to the LECs $c_{i}$, see Eq. (2.17), the leading electromagnetic vertex proportional to the LEC $f_{2}$, see Eq. (2.31), \#5 or the (poorly known) leading charge-dependance of the pion-nucleon coupling constant (212). The resulting IB potentials involve the class-II and III central, tensor and spin-spin components. The CIB potentials typically have the strength of a few $10 \mathrm{keV}$ at relative distances $r \sim M_{\pi}^{-1}$. The CSB tensor and spin-spin potentials are weaker $(<10 \mathrm{keV})$ while the CSB central potential is comparable in size to the CIB contributions. Similarly to the isospin-conserving $2 \pi$-exchange potential, the subleading contributions turn out to be numerically large in comparison to the leading-order ones. In particular, for the class-III central $2 \pi$-exchange potential one obtains $V_{2 N}^{2 \pi,(5)} / V_{2 N}^{2 \pi,(4)} \simeq 3$ for $r \sim M_{\pi}^{-1}$. The main reason for this unpleasant convergence pattern is the same as in the isospin-conserving case and can be traced back to the (large) $\Delta$-isobar contributions to the LECs $c_{3,4}$. We will discuss this issue in more detail in section II.D. Last but not least, there are also numerous IB contact interactions with up to two derivatives involving class-II, III and IV terms, see also (217). The corrections to the 3NF at $\mathrm{N}^{4} \mathrm{~L} \emptyset$ are worked out in Refs. $(211 ; 218)$. At this order, the first IB but charge symmerty conserving 3NFs show up which result from the neutral-to-charged pion mass difference in the $\mathrm{N}^{2} \mathrm{LO} 2 \pi$ - and $1 \pi$-exchange diagrams in the second line of Fig. 13 and the $2 \pi$-exchange diagram involving the $\pi \pi N N$ vertex $\propto f_{1}$. In addition, there are CSB $3 \mathrm{NFs}$ of the $2 \pi$ - and $1 \pi$-exchange types driven by the electromagnetic nucleon mass shift. Again, the strongest 3NFs turn out to be the ones which

\#5 The two other LECs $f_{1,3}$ do not contribute to the $2 \pi$-exchange $2 \mathrm{NF}$ at this order. are proportional to the LECs $c_{3,4}$. They are chargesymmetry conserving and arise from a single insertion of $\delta M_{\pi}^{2}$ into the pion propagators of the $2 \pi$-exchange $3 \mathrm{~N}$ graph. The expected strength of such IB potentials is $\sim 2 \delta M_{\pi}^{2} / M_{\pi}^{2} \sim 13 \%$ as compared to the isospin-invariant ones given in Eq. (2.22) which are known to yield about $\sim 500 \ldots 1000 \mathrm{keV}$ to the triton binding energy (the precise numbers are renormalization scheme dependent). Also the strength of the corresponding coordinate-space potentials, e.g. $\left|g_{A}^{2} \delta M_{\pi}^{2} M_{\pi}^{4} c_{3} /\left(64 \pi^{2} F_{\pi}^{4}\right)\right| \sim 70 \mathrm{keV}$ (here we picked out one particular term), indicates that the resulting IB effects in few-nucleon observables might be sizeable. The CSB 3NFs, on the other hand, do show a more natural convergence pattern and are considerably waeker. Their contribution to e.g. the ${ }^{3} \mathrm{H}-{ }^{3} \mathrm{He}$ binding energy difference is expected to be of the order $\sim 10$ $\mathrm{keV}$.

Recently, a certain classes of even higher-order contributions have been worked out by Kaiser. In particular, he calculated the subleading $\pi \pi \gamma$-exchange potentials proportional to the LECs $c_{3,4}$ at the 2-loop level which (formally) contribute at order $\mathrm{N}^{6} \mathrm{~L} \emptyset \quad(236 ; 237)$. Especially the contributions driven by the LEC $c_{3}$ were found to generate astonishingly strong CSB and CIB potentials which amount to $\sim 1 \%$ of the strongly attractive isoscalar central potential at $\mathrm{N}^{2} \mathrm{LO}$ and reach a few $100 \mathrm{~s}$ of $\mathrm{keV}$ at $r \sim M_{\pi}^{-1}$. Notice, however, that effects of these very strong potentials in $\mathrm{S}$-, $\mathrm{P}$ - and $\mathrm{D}$-waves may, to some extent, be compensated by the corresponding IB contact interactions. The effects in higher partial waves are presumably suppressed due to the shorter range of the $2 \pi$-exchange potential compared with the $1 \pi$-exchange one.

\section{Chiral EFT for few nucleons: applications}

We now turn our attention to applications. As discussed in the previous section, the two-nucleon chiral potential involves the long-range contributions due to the multiple pion exchanges and short-range ones parametrized by contact interactions. Both kinds of terms typically grow with increasing nucleon momenta and become meaningless in the large-momentum region as follows from the very nature of EFT being the low-momentum expansion. As a consequence, the Schrödinger equation is ultraviolet divergent and needs to be regularized (and renormalized). The problem of renormalization in the nonperturbative regime in the context of both pion-less $(41 ; 238-245)$ and pion-full EFT $(139 ; 140 ; 168 ; 246-266)$ has attracted a lot of interest in the past years. The standard procedure to renormalize the Lippmann-Schwinger (LS) equation is based on Wilson's method and implies the following two steps (246). First, one solves the LS equation regularized with the finite momentum (or coordinate-space) cutoff and using the potential truncated at a given order in the chiral expansion as the kernel. Secondly, the LECs accompany- 
ing the contact terms in the potential are determined by matching the resulting phase shifts to experimental data which, in this framework, can be viewed as renormalization. Notice that iterating the truncated expression for the chiral potential in the LS equation necessarily generates ultraviolet divergencies in the Neumann series which require counterterms beyond the given approximation for the potential. As a consequence, taking the limit of the infinite cutoff in such a manifestly nonrenormalizable (in the above mentioned sense) approach might result e.g. in impossibility to resolve the (nonlinear) matching conditions for the corresponding LECs. A detailed discussion on the choice of ultraviolet cutoff and its role in renormalization of the Schrödinger equation is given by Lepage $(246 ; 247)$. He argued that the coordinate-space (momentum-space) cutoff should not be decreased (increased) beyond the separation scale, after which the description of the data stops to improve. Taking the cutoff near this separation scale is the most efficient choice. This strategy has been followed by the currently most advanced $\mathrm{N}^{3} \mathrm{LO}$ analyses of the $2 \mathrm{~N}$ system of Refs. $(271 ; 272)$ where the cutoffs $\Lambda=450 \ldots 600$ $\mathrm{MeV}$ have been employed. These studies were critisized by Nogga et al. (139) who considered low NN partial waves based on the $1 \pi$-exchange potential and contact interactions using a much bigger cutoff variation with $\Lambda<4 \mathrm{GeV}$. They found that higher-order counterterms have to be promoted to LO in the ${ }^{3} P_{0},{ }^{3} P_{2^{-}}{ }^{3} F_{2}$ and ${ }^{3} D_{2}$ channels in order to stabilize the amplitude. On the other hand, the efficiency of such a modified power counting framework was questioned in Ref. (258), where it has been demonstrated that increasing the cutoff and promoting counterterms as suggested in Ref. (139) does not improve the overall description of the scattering observables. For more discussions on the conceptual issues related to the power counting in the $\mathrm{NN}$ system the reader is referred to $(139 ; 140 ; 168 ; 246 ; 247 ; 254 ; 258 ; 262)$. More work is needed in the future in order to clarify the relation between the well-established chiral expansion of the nuclear potential and the scattering amplitude.

We further emphasize that it is possible to nonperturbatively renormalize the partial-wave-projected LS equation with singular $1 / r^{n}$-potentials $(112 ; 262 ; 267-270)$. This program was applied to different NN channels based on the $1 \pi$ - and $2 \pi$-exchange potentials at various orders in the chiral expansion by the Granada group $(252 ; 253 ; 255-257 ; 260 ; 263 ; 264)$. In these studies, the short-range counter terms are replaced by adjustable parameters entering the short-distance boundary conditions. The number of such parameters in each channel is uniquely determined by the sign (attractive vs. repulsive) of the strongest singularity which raises concerns about a systematic improvability (in the EFT sense) of such a framework. Nevertheless, the findings of these studies in attractive channels provide an impressive demonstration of the existence of the long-range correlations in the $\mathrm{NN}$ scattering observables.

The most advanced analyses of the two-nucleon sys-
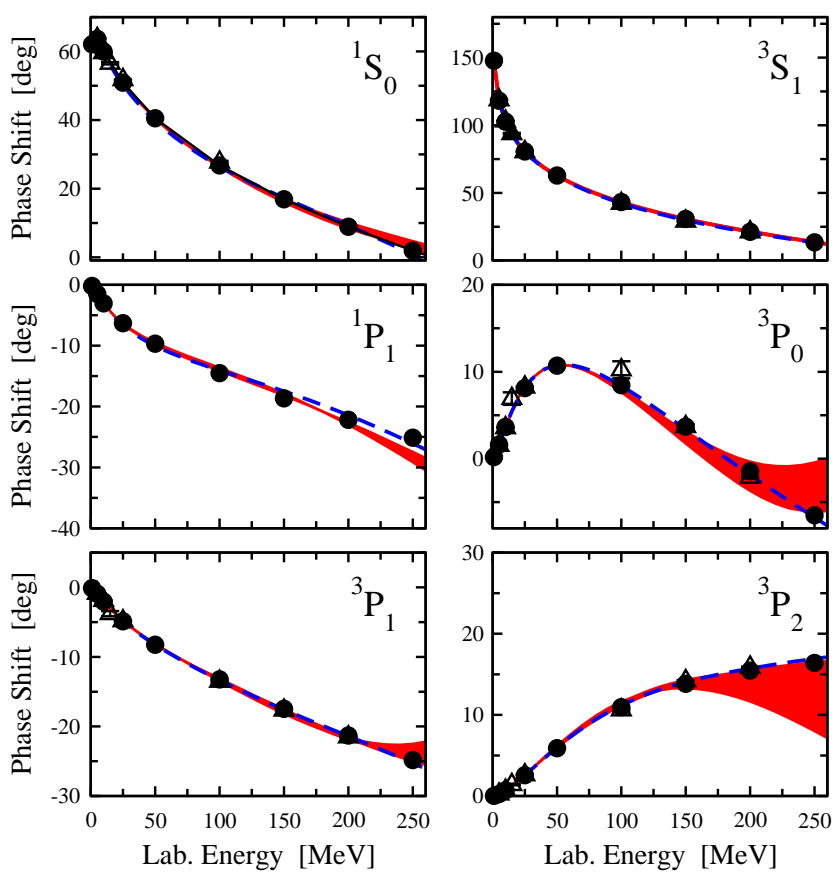

FIG. 15 Neutron-proton phase shifts in S- and P-waves at $\mathrm{N}^{3} \mathrm{LO}$ in comparison with the Nijmegen $(273 ; 274)$ (filled circles) and Virginia Tech (275) (open triangles) PWA. Shaded bands (dashed lines) refer to the calculations by EGM (272) (EM (271)).

tem based on the Weinberg power counting take into account the $2 \mathrm{NF}$ contributions up to $\mathrm{N}^{3} \mathrm{LO}(271 ; 272)$. Most of the LECs $c_{i}, d_{i}$ entering the long-range part of the potential are sufficiently well determined in the pionnucleon system (184). \#6 The $24^{\# 7}$ unknown LECs entering the short-range part of the $2 \mathrm{NF}$ at $\mathrm{N}^{3} \mathrm{LO}$ have been extracted from the low-energy $\mathrm{NN}$ data for several choices of the cutoff in the Schrödinger equation. Both $\mathrm{N}^{3} \mathrm{LO}$ potentials of Entem and Machleidt (271) (EM) and Epelbaum, Glöckle and Meißner (272) (EGM) yield accurate results for the neutron-proton phase shifts up to $E_{\text {lab }} \sim 200 \mathrm{MeV}$ and the deuteron observables. This is exemplified in Figs. 15, 16 where the EGM and EM results for the neutron-proton S- P- and D-waves and the corresponding mixing angles are shown in comparison with PWA results from Refs. (273-275). The bands in the EGM analysis result from the variation of the cutoff in the LS equation (spectral function regularization) in the range $\Lambda=450 \ldots 600 \mathrm{MeV}(\tilde{\Lambda}=500 \ldots 700 \mathrm{MeV})$. It is comforting to see that in most cases the results of both analyses agree with each other within the estimated theoretical uncertainty. Notice, however, that the EM

\footnotetext{
\#6 Notice, however, that the value of the LEC $c_{4}$ adopted in (271), $c_{4}=5.4 \mathrm{GeV}^{-1}$ is not compatible with pion-nucleon scattering where one finds at order $Q^{3}$ (185): $c_{4}=3.40 \pm 0.04 \mathrm{GeV}^{-1}$.

\#7 This number refers to isospin-invariant contact interactions.
} 

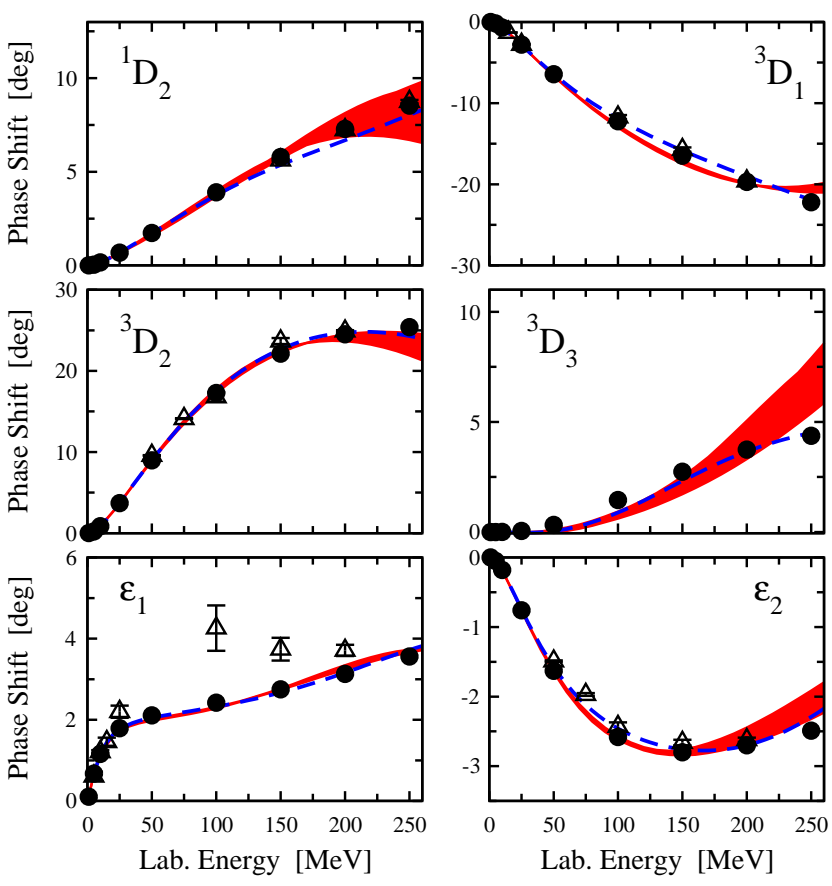

FIG. 16 Neutron-proton phase shifts in D-waves and the mixing angles $\epsilon_{1,2}$ at $\mathrm{N}^{3} \mathrm{LO}$. For notation see Fig. 15.

and EGM analyses differ from each other in several important aspects. For example, the so-called spectral function regularization $(155 ; 156)$ of the $2 \pi$-exchange contributions has been adopted by EGM while the analysis by EM is based upon dimensionally regularized expressions. Further differences can be attributed to the implementation of the momentum-space cutoff in the Schrödinger equation and the treatment of relativistic effects. More precisely, the work by EGM is based on the "relativistic" Schrödinger/Lippmann-Schwinger equation, a natural extension of the usual nonrelativistic equations utilizing the relativistic relation between the CMS energy and momentum, see (170) for more details. This equation can be straightforwardly generalized to the case of several nucleons, see (276-278) for recent studies of relativistic effects in $3 \mathrm{~N}$ observables, and can also be cast into equivalent nonrelativistic-like forms $(170 ; 279)$ (provided the potential is appropriately modified). On the other hand, the analysis of Ref. (271) is based on the nonrelativistic Schrödinger equation and uses the static $1 \pi$ exchange potential and the $1 / \mathrm{m}$ - and $1 / \mathrm{m}^{2}$-corrections to the $2 \pi$-exchange potential from Refs. $(142 ; 173)$, where no particular dynamical equation is specified. It is unclear whether the relativistic corrections to the potential used in (271) are consistent with the nonrelativistic Schrödinger equation. Further differences between the EGM and EM analyses result from the fitting procedure: the LECs accompanying the contact interactions were determined by EM/EGM by fitting directly to the scattering data/to the Nijmegen PWA $(273 ; 274)$. For this reason, EGM adopted the same treatment of IB effects as followed by the Nijmegen group and did not include

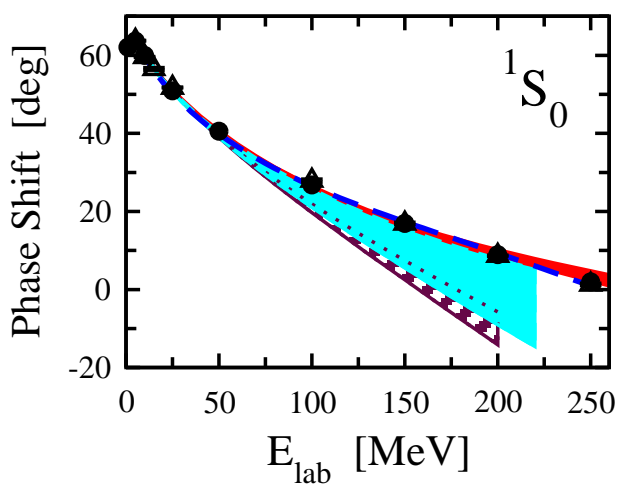

FIG. 17 Neutron-proton ${ }^{1} \mathrm{~S}_{0}$ partial wave at NLO (dashed band), $\mathrm{N}^{2} \mathrm{LO}$ (light-shaded band) and $\mathrm{N}^{3} \mathrm{LO}$ (dark-shaded band) in comparison with the Nijmegen $(273 ; 274)$ (filled circles) and Virginia Tech (275) (open triangles) PWA.

e.g. the leading IB contributions to the $2 \pi$-exchange potential. Perhaps, the most important difference between the two studies is related to the estimation of the theoretical uncertainty. In the work by EGM, the theoretical uncertainty was estimated by varying the cutoffs in the Schrödinger equation and the spectral function representation for the $2 \pi$-exchange potential restricted by the condition that the resulting LECs are of a natural size which might be viewed as a self-consistency check for calculations carried out within the power counting scheme based on naive dimensional analysis, see $(87 ; 163)$. Notice further that at $\mathrm{NLO}$ and $\mathrm{N}^{2} \mathrm{LO}$, the strengths of various contact interactions are well understood in terms of resonance saturation on the basis of phenomenological one-boson exchange models (280). No serious attempt to provide a realistic error estimation was done in the analysis of EM. On the other hand, their work clearly demonstrates that for a particularly chosen regularization prescription it is even possible to accuratly describe two-nucleon scattering data for $E_{\text {lab }}>200 \mathrm{MeV}$. For further technical details, results for various scattering observables and the properties of the deuteron the reader is referred to the original publications $(271 ; 272)$ and the review article (163).

To illustrate the convergence of the chiral expansion for NN phase shifts, we show in Fig. 17 the results for the ${ }^{1} S_{0}$ partial wave at NLO, $\mathrm{N}^{2} \mathrm{LO}$ (156) and $\mathrm{N}^{3} \mathrm{LO}$ (272). We emphasize that the variation of the cutoff at both $\mathrm{NLO}$ and $\mathrm{N}^{2} \mathrm{LO}$ only shows the effects of missing $\mathrm{N}^{3} \mathrm{LO}$ contact interactions. It, therefore, does not provide a realistic estimation of the theoretical uncertainty at NLO, see (163) for an extended discussion.

Applications to the three-nucleon system have so far been carried out up to $\mathrm{N}^{2} \mathrm{LO}$. At NLO, no $3 \mathrm{NF}$ needs to be taken into account. This allowed for a parameterfree predictions of various $3 \mathrm{~N}$ scattering observables at low energies as well as for the triton and $\alpha$-particle binding energies (122). Using the most recent version of the 
NLO potential based on the spectral functions regularization, one finds at NLO $(163) B_{3 \mathrm{H}}=7.71 \ldots 8.46 \mathrm{MeV}$ and $B_{3} \mathrm{He}=24.38 \ldots 28.77 \mathrm{MeV}$ to be compared with the experimental values $B^{3} \mathrm{H}=8.482 \mathrm{MeV}$ and $B^{{ }^{3} \mathrm{He}}=$ 28.30 MeV. These numbers are similar to the ones obtained in Ref. (122) within the framework based on dimensional regularization.

At $\mathrm{N}^{2} \mathrm{LO}$ one, for the first time, has to take into account the crresponding 3 NFs. The two LECs $D$ and $E$ entering the expressions for the $3 \mathrm{NF}$ in Eq. (2.22) have been determined by fitting the ${ }^{3} \mathrm{H}$ binding energy and either the $n d$ doublet scattering length (123), the ${ }^{4} \mathrm{He}$ binding energy (281) or the properties of light nuclei (282). Notice that the $\pi N N N N$ vertex entering the $1 \pi$-exchange-contact $3 \mathrm{NF}$ also plays an important role in processes with a completely different kinematics such as e.g. the pion production in the NN collisions (283), see section II.E, or weak reactions like $p p \rightarrow d e^{+} \nu_{e}$, see (284) and references therein. This offers the possibility to extract the corresponding LEC from these processes, see (284) for a recent attempt. With the LECs being determined as described above, the resulting nuclear Hamiltonian can be used to describe the dynamics of few-nucleon systems. In particular, $3 \mathrm{~N}$ continuum observables offer a natural and rich testing ground for the chiral forces. In Refs. $(122 ; 123 ; 285-293)$ various $3 \mathrm{~N}$ scattering observables have been explored by solving the momentum-space Faddeev equations with chiral twoand three-nuclein forces as input. In the formulation of Ref. (22), one first computes the $T$-matrix by solving the Faddeev-like integral equation

$$
\begin{aligned}
T & =t P \phi+\left(1+t G_{0}\right) V_{3 N}^{1}(1+P) \phi+t P G_{0} T \\
& +\left(1+t G_{0}\right) V_{3 N}^{1}(1+P) G_{0} T
\end{aligned}
$$

where the initial state $\phi$ is composed of a deuteron and a momentum eigenstate of the projectile nucleon. Here $V_{3 N}^{i}$ is that part of the $3 \mathrm{~N}$ force which singles out the particle $i$ and which is symmetric under the interchange of the two other particles. The complete $3 \mathrm{NF}$ is decomposed as $V_{3 N}=V_{3 N}^{1}+V_{3 N}^{2}+V_{3 N}^{3}$. Further, $G_{0}=1 /\left(E-H_{0}\right)$ is the free propagator of the nucleons, $P$ is a sum of a cyclical and anti-cyclical permutation of the three particles and $t$ denotes the two-body $t$-matrix. Once $T$ is calculated, the transition operators $U_{\mathrm{el}}$ and $U_{\mathrm{br}}$ for the elastic and break-up channels can be obtained via

$$
\begin{aligned}
U_{\mathrm{el}} & =P G_{0}^{-1}+P T+V_{3 N}^{1}(1+P)\left(1+G_{0} T\right), \\
U_{\mathrm{br}} & =(1+P) T .
\end{aligned}
$$

For details on solving these equations in momentum space using a partial wave decomposition the reader is referred to (294). The partial wave decomposition of the $1 \pi$-exchange and contact $3 \mathrm{NF}$ at $\mathrm{N}^{2} \mathrm{LO}$ and the onepion-two-pion-exchange topology at $\mathrm{N}^{3} \mathrm{LO}$ is detailed in Refs. (123) and (295), respectively. The expressions for various observables in terms of the transition operators are given in (22). The inclusion of the long-range electromagnetic interaction requires a non-trivial generalization
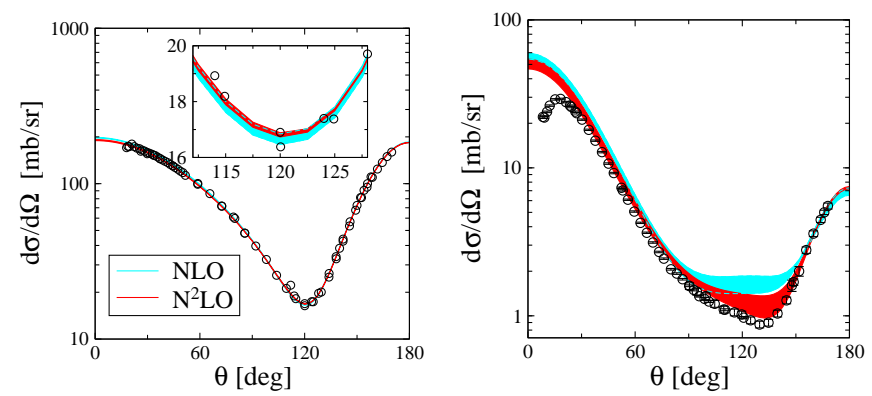

FIG. 18 Differential cross section for elastic $n d$ scattering at $E_{\text {lab }}=10 \mathrm{MeV}$ (left panel) and $65 \mathrm{MeV}$ (right panel). Light (dark) shaded bands depict the results at NLO $\left(\mathrm{N}^{2} \mathrm{LO}\right)$. The neutron-deuteron data at $10 \mathrm{MeV}$ are from Ref. (298). The remaining data at $10 \mathrm{MeV}$ are the Coulomb/IB-corrected proton-deuteron data from Refs. (299-301). The data at $65 \mathrm{MeV}$ are proton-deuteron data from Ref. (302).

of the formalism, see $(296 ; 297)$ for recent progress along this line.

The results for the differential cross section in elastic $n d$ scattering are in a good agreement with the data, see Fig. 18 for two representative examples. Notice, however, that the theoretical uncertainty becomes significant already at intermediate energies. Qualitatively, this behavior is consistent with the one observed in the twonucleon system (272). Notice further that the description of the data improves significantly when going from NLO to $\mathrm{N}^{2} \mathrm{LO}$. The situation is similar for vector and tensor analyzing powers, see Ref. (163) for a recent review article. More complicated spin observables have also been studied. As a representative example, we show in Fig. 19 a selection of the proton-to-proton and protonto-deuteron polarization transfer coefficients measured in $d(\vec{p}, \vec{p}) d$ and $d(\vec{p}, \vec{d}) p$ reactions at $E_{p}^{\text {lab }}=22.7 \mathrm{MeV}$ $(303 ; 304)$. The results at $\mathrm{N}^{2} \mathrm{LO}$ are in a reasonable agreement with the data, see (302) for more examples. One further observes that the theoretical uncertainty obtained by the cutoff variation is underestimated at NLO, see the discussion earlier in the text. It is, however, comforting to see that the description of the data improves significantly when going from NLO to $\mathrm{N}^{2} \mathrm{LO}$.

The nucleon-deuteron breakup reaction offers even more possibilities than the elastic channel due to the much richer kinematics corresponding to three nucleons in the final state. It has also been studied extensively over the last years, both theoretically and experimentally, leaving one with mixed conclusions. While the differential cross section in some configurations such as e.g. the recently measured $n p$ final-state interaction, co-planar star and an intermediate-star geometries at low energies are in a very good agreement with the data (287), large deviations are observed in certain other configurations. In particular, the so-called symmetric space-star configuration (SST) appears rather puzzling. In this configuration, the plane in the CMS spanned by the outgoing nu- 

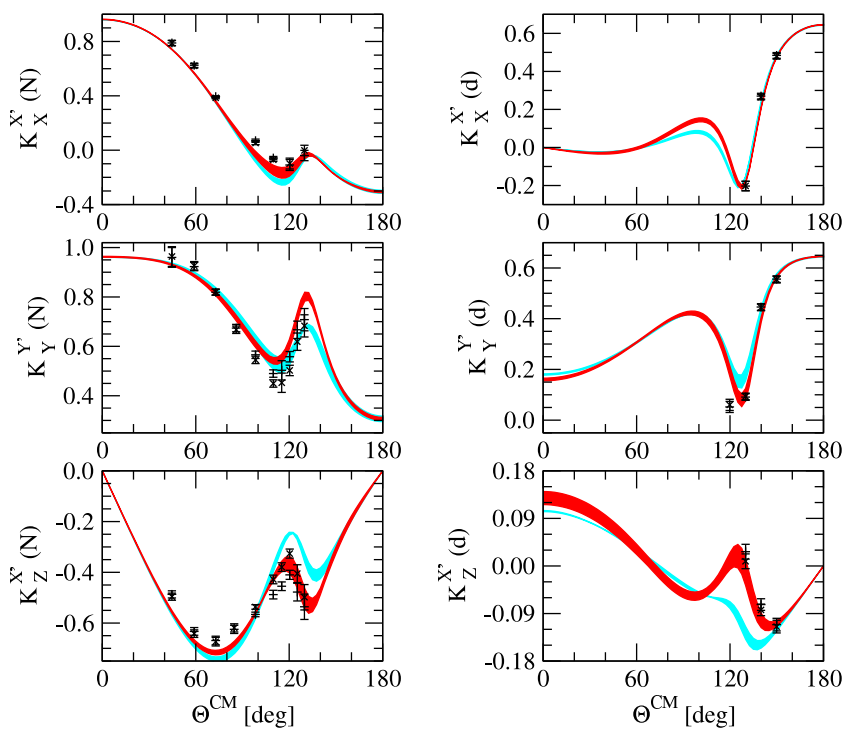

FIG. 19 The proton-to-proton (left panel) and proton-todeuteron (right panel) polarization transfer coefficients in $d(\vec{p}, \vec{p}) d$ and $d(\vec{p}, \vec{d}) p$ reactions at $E_{p}^{\text {lab }}=22.7$. Light (dark) shaded bands depict the results at NLO $\left(\mathrm{N}^{2} \mathrm{LO}\right)$. Data are from Refs. $(303 ; 304)$.
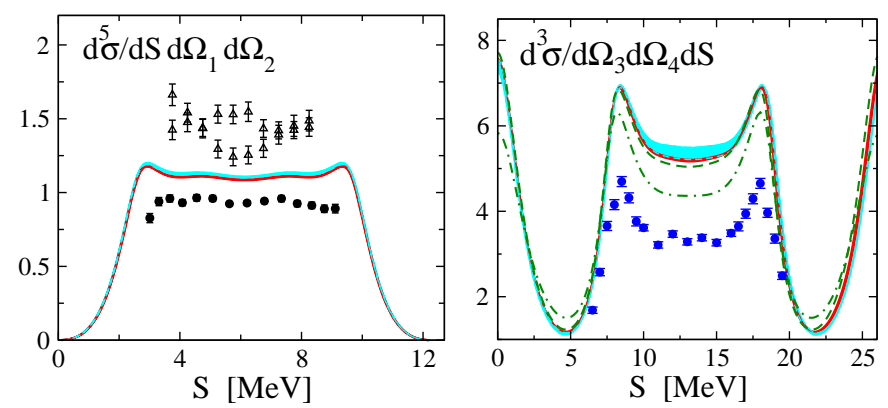

FIG. 20 Chiral EFT predictions for neutron-deuteron breakup cross section (in $\mathrm{mb} \mathrm{MeV}^{-1} \mathrm{sr}^{-2}$ ) along the kinematical locus $S$. Light-shaded (dark-shaded) bands refer to the results at NLO $\left(\mathrm{N}^{2} \mathrm{LO}\right)$. Left panel: The SST configuration at $E_{N}=13 \mathrm{MeV}$. Neutron-deuteron data (open triangles) are from $(305 ; 306)$, proton-deuteron data (filled circles) are from (307). Right panel: The SCRE configuration with $\alpha=56^{\circ}$ at $E_{N}=19 \mathrm{MeV}$ (292). Dashed and dashed-dotted lines are results based on the CD Bonn 2000 2NF (18) combined with the TM99 3NF (308) and the coupled channel calculation including the explicit $\Delta$ and the Coulomb interaction (296), respectively.

cleons is perpendicular to the beam axis, and the angles between the nucleons are $120^{\circ}$. At $E_{\text {lab }}=13 \mathrm{MeV}$, the proton-deuteron and neutron-deuteron $(n d)$ cross section data deviate significantly from each other. Theoretical calculations based on both phenomenological and chiral nuclear forces have been carried out for the $n d$ case and are unable to describe the data, see Fig. 20. Moreover, the Coulomb effect was found to be far too small to ex- plain the difference between the $p d$ and $n d$ data sets. Recently, proton-deuteron data for a similar symmetric constant relative-energy (SCRE) configuration have been measured in Cologne (292). This geometry is characterized by the angle $\alpha$ between the beam axis and the plane in the CMS spanned by the outgoing nucleons. Similar to the SST geometry, one observes large deviations between the theory and the data, in particular for $\alpha=56^{\circ}$, see Fig. 20. The included 3NFs have little effect on the cross section while the effect of the Coulomb interaction is significant and removes a part of the discrepancy. Notice that all above cases correspond to rather low energies where one expects good convergence of the chiral expansion. Furthermore, contrary to the $A_{y}$-puzzle, the cross sections discussed above are mainly sensitive to the twonucleon S-waves without any known fine tuning between partial waves. First attempts have been made in the past few years to perform deuteron breakup experiments at intermediate energies, in particular at $E_{N}=65 \mathrm{MeV}$ (289), in which a large part of the phase space is covered at once. Chiral EFT results at $\mathrm{N}^{2} \mathrm{LO}$ for more than 155 data points were shown to be of a comparable quality to the ones based on modern phenomenological nuclear forces.

Recently, first results for the $4 \mathrm{~N}$ continuum based on both phenomenological and chiral nuclear forces and including the Coulomb interactions have become available, see $(309 ; 310)$ for $p-{ }^{3} \mathrm{He}$ scattering, (311) for the $n-{ }^{3} \mathrm{He}$, $p-{ }^{3} \mathrm{H}$ and $d-d$ scattering, and (312) for the related earlier work. These studies do not yet include effects of $3 \mathrm{NFs}$ but clearly indicate that at least some of the puzzles observed in the $3 \mathrm{~N}$ continuum also persist in the $4 \mathrm{~N}$ continuum (such as e.g. the $A_{y}$-puzzle in $p-{ }^{3}$ He scattering (310)). For a promising new approach to describe scattering states in even heavier systems the reader is referred to (313).

The properties of certain S-shell and P-shell nuclei with $A \leq 13$ have been analyzed recently based on the no-core shell model (NCSM), see $(281 ; 282)$ and (314) for an overview. In Fig. 21 we show some results from Ref. (282) for the spectra of ${ }^{10} \mathrm{~B},{ }^{11} \mathrm{~B},{ }^{12} \mathrm{C}$ and ${ }^{13} \mathrm{C}$. We emphasize that the LECs $D$ and $E$ entering the $\mathrm{N}^{2} \mathrm{LO}$ $3 \mathrm{NF}$ were determined in these calculations by the triton binding energy and a global fit to selected properties of ${ }^{6} \mathrm{Li},{ }^{10} \mathrm{~B}$ and ${ }^{12} \mathrm{C}$. These studies clearly demonstrate that the chiral $3 \mathrm{NF}$ plays an important role in the description of spectra and other properties of light nuclei. The inclusion of the $3 \mathrm{NF}$ allows to considerably improve the agreement with the data. Further results for light nuclei and the dilute neutron matter based on the lattice formulation of chiral EFT are given in sections II.G and III.E.

\section{The role of the $\Delta$-isobar}

The chiral expansion for the long-range part of the nuclear force discussed in the previous section exhibits a 

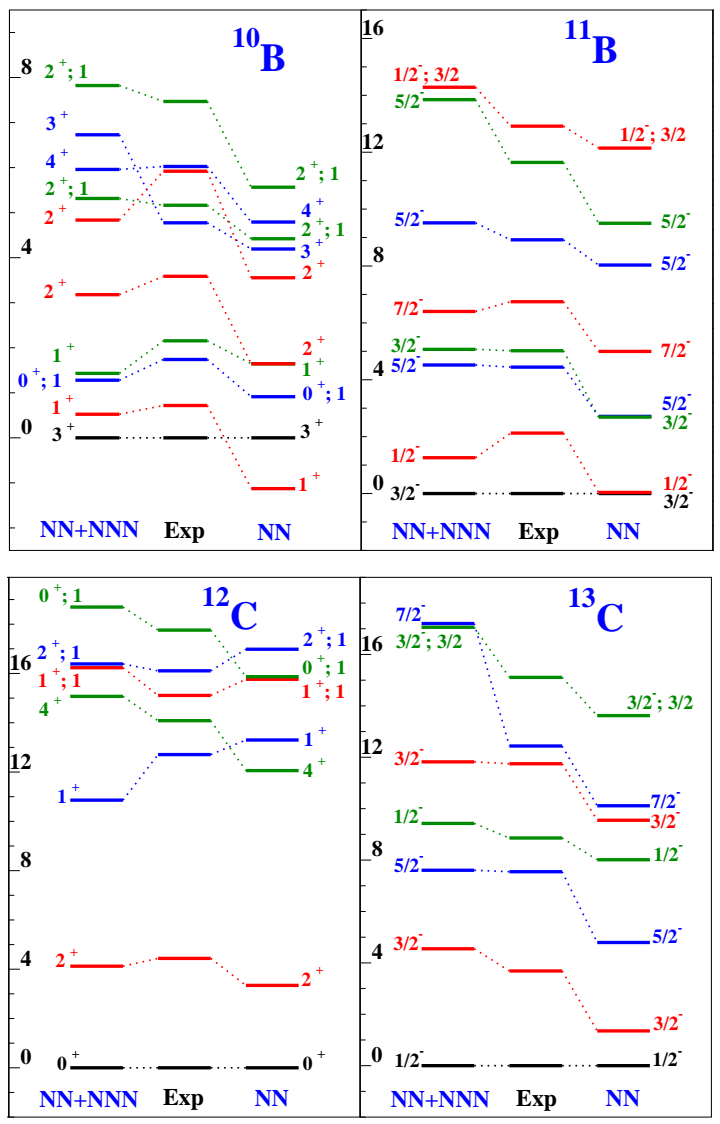

FIG. 21 States dominated by P-shell configurations for ${ }^{10} \mathrm{~B}$, ${ }^{11} \mathrm{~B},{ }^{12} \mathrm{C}$ and ${ }^{13} \mathrm{C}$. The excitation energy scales are in $\mathrm{MeV}$. Calculation is carried out in the framework of NCSM based on chiral $\mathrm{N}^{3} \mathrm{LO} 2 \mathrm{NF}$ of Ref. (172) and $\mathrm{N}^{2} \mathrm{LO} 3 \mathrm{NF}$. For more details on the calculation see (282). Figure courtesy of Petr Navratil.

somewhat unnatural convergence pattern in certain cases such as e. g. for the central part of the $2 \pi$-exchange potential. The origin of the unnaturally strong subleading contribution in this case potential can be traced back to the large values of the dimension-two low-energy constants (LECs) $c_{3,4}$ which are also responsible for the numerical dominance of the subleading $3 \pi$-exchange (180) and charge-symmetry breaking $2 \pi$-exchange $2 \mathrm{NF}(212)$ over the corresponding leading contributions. The large values of these LECs are well understood in terms of resonance saturation (160). In particular, the $\Delta(1232)$ provides the dominant (significant) contribution to $c_{3}\left(c_{4}\right)$. Given its low excitation energy, $\Delta \equiv m_{\Delta}-m=293 \mathrm{MeV}$, and strong coupling to the $\pi N$ system, the $\Delta$-isobar is known to play an important role in nuclear physics. One can, therefore, expect that the explicit inclusion of $\Delta$ in EFT will allow to resum a certain class of important contributions and improve the convergence as compared to the delta-less theory, provided a proper power counting scheme such as the small scale expansion (SSE) (315) is employed, see also (316). The SSE is a phenomenologi- cal extension of chiral perturbation theory in which the small expansion parameter includes external momenta, pion masses and the nucleon-delta mass splitting,

$$
Q / \Lambda \in\left\{p / \Lambda, M_{\pi} / \Lambda, \Delta / \Lambda\right\}
$$

i.e. the delta-nucleon mass splitting is treated as $\Delta \equiv$ $m_{\Delta}-m \sim M_{\pi}$ rather than $\Delta \sim \Lambda \gg M_{\pi}$. The improved convergence has been explicitly demonstrated for pionnucleon scattering where the description of the phase shifts at third order in the SSE comes out superior (inferior) to the third (fourth) order chiral expansion in the delta-less theory (319). In the following, we will overview the additional contributions to the nuclear force which arise in the $\Delta$-full theory as compared to the $\Delta$-less theory and discuss the implications for the convergence of the low-momentum expansion. Notice that in such a setting we do not need to consider the $N N \rightarrow N \Delta$, $N N \rightarrow \Delta \Delta$ and $N \Delta \rightarrow \Delta \Delta$ transitions which would correspond to the coupled-channel approach. The adopted counting rules for the nucleon mass and the delta-nucleon mass difference imply for the momentum scale associated with real delta production $\sqrt{m \Delta} \sim \sqrt{m M_{\pi}} \sim \Lambda$. For typical external momenta (energies) of the nucleons of the order $|\vec{p}| \sim M_{\pi}\left(E_{\text {kin }} \sim M_{\pi}^{2} / m\right)$ we are interested in, the momenta associated with real delta production can be safely integrated out. We, therefore, only need to consider the contributions to the nuclear force arising due to virtual delta excitations.

The effective Lagrangian can be straightforwardly extended to include the $\Delta$-degrees of freedom. To work out $\Delta$-contributions up to $\mathrm{N}^{2} \mathrm{LO}$, the following additional terms in the heavy-baryon Lagrangian have to be taken into account:

$$
\begin{aligned}
\mathcal{L}_{\pi \Delta}^{(0)} & =-\bar{T}_{\mu}^{i}\left[i v \cdot D^{i j}-\Delta \delta^{i j}\right] g^{\mu \nu} T_{\nu}^{j}+\ldots, \\
\mathcal{L}_{\pi N \Delta}^{(0)} & =h_{A} \bar{T}_{\mu}^{i} P^{\mu \nu} \omega_{\nu}^{i} N+\text { h.c. } \\
\mathcal{L}_{\pi N \Delta}^{(1)} & =\left(b_{3}+b_{8}\right) \bar{T}_{\mu}^{i} i P^{\mu \nu} \omega_{\nu \rho}^{i} v^{\rho} N+\text { h.c. }+. .
\end{aligned}
$$

where $T_{\mu}^{i}$ with $\mu(i)$ being the Lorentz (isospin) index denotes the large component of the delta field. Further, $D_{\mu}^{i j}$ refers to the chiral covariant derivative for the delta fields and $P_{\mu \nu}$ is the standard projector on the $3 / 2$ components, $P_{\mu \nu}=g_{\mu \nu}-v_{\mu} v_{\nu}-4 S_{\mu} S_{\nu} /(1-d)$, with $d$ being the number of space-time dimensions. We also have $w_{\alpha}^{i}=\left\langle\tau^{i} u_{\alpha}\right\rangle / 2$ and $w_{\alpha \beta}^{i}=\left\langle\tau^{i}\left[\partial_{\alpha}, u_{\beta}\right]\right\rangle / 2$. The only relevant LEC in the lowest-order Lagrangian is the $\pi N \Delta$ axial coupling $h_{A}$. At subleading order, the combination of $\pi N \Delta$ LECs $b_{3}+b_{8}$ contributes. For more details on the notation, the reader is referred to $(315 ; 319)$, see also (38) for a recent review article and $(317 ; 318)$ for different formulations. Finally, it should also be emphasized that the only possible derivative-less $N N N \Delta$ contact interaction

$$
\mathcal{L}_{N \Delta}^{(0)} \propto\left(\bar{T}_{i}^{\mu} N \bar{N} S_{\mu} \tau^{i} N+\text { h. c. }\right)
$$

vanishes due to the Pauli principle (320). 
The values of the LECs in the $\pi N$ Lagrangian are, clearly, different in the $\Delta$-less and $\Delta$-full theories and can be naturally extracted from $\pi N$ scattering, see (319) for such a determination at the leading one-loop level (i.e. order $Q^{3}$ ). At subleading order, which is sufficient for our purpose, the determination of $c_{i}$ from the $\pi N \mathrm{~S}$ and $\mathrm{P}$-wave threshold coefficients yields in the delta-less theory (321)

$$
c_{1}=-0.57, c_{2}=2.84, c_{3}=-3.87, c_{4}=2.89,
$$

where only central values are given and the units are $\mathrm{GeV}^{-1}$. The above values are somewhat smaller in magnitude than the ones obtained at higher orders, see e.g. (184). Including the contributions form the $\Delta$, one finds

$$
\begin{aligned}
& c_{1}=-0.57, c_{2}=-0.25, c_{3}=-0.79, c_{4}=1.33 \\
& b_{3}+b_{8}=1.40
\end{aligned}
$$

Notice that the LECs $c_{2,3,4}$ are strongly reduced in magnitude when the $\Delta$-isobar is included. It should also be emphasized that the values of these LECs depend sensitively on the choice of $h_{A}$, which in the above case was set to $h_{A}=3 g_{A} /(2 \sqrt{2})$ from $\mathrm{SU}(4)$ (or large $N_{c}$ ). The results for the threshold coefficients and the $2 \pi$ exchange potential are, however, rather stable (321). We also emphasize that the description of the P-wave threshold parameters improves significantly upon inclusion of the delta-isobar.

We are now in the position to discuss the leading and subleading contributions of the $\Delta$-isobar to the nuclear force. Since the appearance of a virtual $\Delta$-isobar requires at least one loop, the corresponding contributions first appear at $\mathrm{NLO}(\nu=2)$. The relevant $2 \mathrm{~N}$ and $3 \mathrm{~N}$ diagrams can be obtained from the ones of Figs. 12 and 13 by replacing the nucleon propagators by the ones of the $\Delta$-fields in all intermediate states. We first discuss the $2 \mathrm{NF}$. Similarly to the $\Delta$-less theory, the additional contributions to the $1 \pi$-exchange potential and contact interactions at both $\mathrm{NLO}$ and $\mathrm{N}^{2} \mathrm{LO}$ only lead to renormalization of various LECs. The $2 \pi$-exchange diagrams were first discussed by Ordóñez et al. (150) using time-ordered perturbation theory. These contributions were then calculated by Kaiser et al. (322) using the Feynman graph technique. The corrections at $\mathrm{N}^{2} \mathrm{LO}$ have been worked out recently (321). We refrain from showing here the resulting expressions which are rather involved and only give the results for the isovector tensor $2 \pi$-exchange potential $W_{T}$, defined according to $V_{2 N}=\boldsymbol{\tau}_{1} \cdot \boldsymbol{\tau}_{2} \vec{\sigma}_{1} \cdot \vec{q} \sigma_{2} \cdot \vec{q} W_{T}$, which may serve as a representative example:

$$
\begin{aligned}
W_{T}^{(2)}= & -\frac{h_{A}^{2}}{1296 \pi^{2} F_{\pi}^{4} \Delta}\left[9 \pi g_{A}^{2} \omega^{2} A^{\tilde{\Lambda}}(q)+h_{A}^{2}\left(2 L^{\tilde{\Lambda}}(q)\right.\right. \\
& \left.\left.+\left(4 \Delta^{2}+\omega^{2}\right) D^{\tilde{\Lambda}}(q)\right)\right]
\end{aligned}
$$

$$
\begin{aligned}
W_{T}^{(3)}= & -\frac{h_{A}^{2} \Delta}{648 \pi^{2} F_{\pi}^{4}}\left[\left(2\left(b_{3}+b_{8}\right) g_{A}\left(\omega^{2}-12 \Delta^{2}\right)\right.\right. \\
& \left.-9 c_{4}\left(\omega^{2}-4 \Delta^{2}\right)\right) D^{\tilde{\Lambda}}(q) \\
& \left.+6\left(3 c_{4}-2\left(b_{3}+b_{8}\right) h_{A}\right) L^{\tilde{\Lambda}}(q)\right]
\end{aligned}
$$

Here, the new loop function $D^{\tilde{\Lambda}}(q)$ is defined via

$$
D^{\tilde{\Lambda}}(q)=\frac{1}{\Delta} \int_{2 M_{\pi}}^{\tilde{\Lambda}} \frac{d \mu}{\mu^{2}+q^{2}} \arctan \frac{\sqrt{\mu^{2}-4 M_{\pi}^{2}}}{2 \Delta} .
$$

The complete results for the $\Delta$-contributions can be found in Refs.(321; 322). It is instructive to verify the consistency between the $\Delta$-full and $\Delta$-less theories which requires that the contributions due to intermediate $\Delta$ excitations, expanded in powers of $1 / \Delta$ can be absorbed into a redefinition of the LECs in the $\Delta$-less theory. This is only possible if the nonpolynomial (in momenta) terms up to $\mathrm{N}^{2} \mathrm{LO}$ resulting from such an expansion have the same form as expressions in Eqs. $(2.14,2.18)$. This indeed turns out to be the case: all expanded nonpolynomial terms up to $\mathrm{N}^{2} \mathrm{LO}$ are exactly reproduced by the shift in the LECs $c_{3,4}$

$$
c_{3}=-2 c_{4}=-\frac{4 h_{A}^{2}}{9 \Delta}
$$

in Eqs. $(2.14,2.18)$.

To get more insight into the strength of various $2 \pi$ exchange contributions in the $\Delta$-full and $\Delta$-less theories, it is useful to switch to coordinate space. The $2 \pi$ exchange potential can then be written as

$$
\begin{aligned}
\tilde{V}(r) & =\tilde{V}_{C}+\boldsymbol{\tau}_{1} \cdot \boldsymbol{\tau}_{2} \tilde{W}_{C}+\left[\tilde{V}_{S}+\boldsymbol{\tau}_{1} \cdot \boldsymbol{\tau}_{2} \tilde{W}_{S}\right] \vec{\sigma}_{1} \cdot \vec{\sigma}_{2} \\
& +\left[\tilde{V}_{T}+\boldsymbol{\tau}_{1} \cdot \boldsymbol{\tau}_{2} \tilde{W}_{T}\right] S_{12}
\end{aligned}
$$

where $S_{12}=3 \vec{\sigma}_{1} \cdot \hat{r} \vec{\sigma}_{2} \cdot \hat{r}-\vec{\sigma}_{1} \cdot \vec{\sigma}_{2}$ is the tensor operator. The scalar functions $\tilde{V}_{i}(r)$ and $\tilde{W}_{i}(r)$ are plotted in Fig. 22 using the values for the LECs specified in Eqs. (2.43) and (2.44). As expected, one observes a more natural convergence pattern in the theory with explicit deltas with the $\mathrm{N}^{2} \mathrm{LO}$ contributions yielding typically only modest corrections to the NLO result. This is, clearly, not the case in the delta-less theory where the entire contributions to $\tilde{V}_{C}$ and $\tilde{W}_{T, S}$ are generated at $\mathrm{N}^{2} \mathrm{LO}$. On the other hand, the $\mathrm{N}^{2} \mathrm{LO} 2 \pi$-exchange potential in the delta-less theory provides a very good approximation to the potential resulting at the same order in the delta-full theory. This indicates that the saturation of the LECs $c_{3,4}$ is the most important effect of the $\Delta$-isobar at the considered order. The results for NN Fand other peripheral waves calculated using the Born approximation also clearly demonstrate the improved convergence in the theory with explicit $\Delta$, see Fig. 23.

As explained in section II.B, the first nonvanishing contributions to the $3 \mathrm{NF}$ appear in the $\Delta$-less theory at $\mathrm{N}^{2} \mathrm{LO}$. The situation is different in the $\Delta$-full theory where the first $3 \mathrm{NF}$ contribution is generated at NLO 

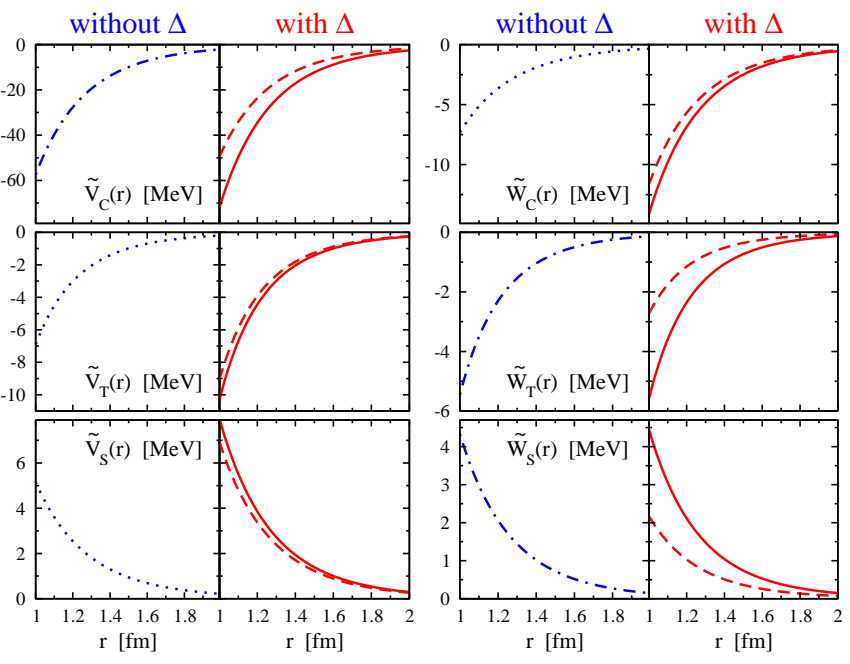

FIG. 22 Isoscalar (left panel) and isovector (right panel) components of the $2 \pi$-exchange potential in coordinate space for $\tilde{\Lambda}=700 \mathrm{MeV}$. Dashed and solid (dotted and dashed-dotted) lines refer to the NLO and $\mathrm{N}^{2} \mathrm{LO}$ results in the delta-full (delta-less) theory, respectively. There are no contributions to $\tilde{V}_{C}$ and $\tilde{W}_{T, S}\left(\tilde{V}_{T, S}\right.$ and $\left.\tilde{W}_{C}\right)$ at NLO $\left(\mathrm{N}^{2} \mathrm{LO}\right)$ in the delta-less theory.

by the second graph in the first line of Fig. 13 with the intermediate nucleon propagator being replaced by the one of the $\Delta$-field. In fact, the importance of the $\pi N P_{33}$ partial wave corresponding to the excitation of the $\Delta$ resonance in the $3 \mathrm{NF}$ has been realized already 50 years by Fujita and Miyazawa (23). The resulting expression for the $\Delta$-contribution to the $2 \pi$-exchange $3 \mathrm{NF}$ is exactly reproduced by the first term in Eq. (2.22) if one uses the $\Delta$ saturation values for the LECs $c_{i}$ from Eq. (2.47). This, in fact, follows from the decoupling theorem and the fact that the static $\Delta$ propagator is proportional to $\Delta^{-1}$. Notice that there are no short-range $3 \mathrm{NFs}$ with intermediate $\Delta$-excitation since the corresponding $N N N \Delta$ interaction is Pauli forbidden. Stated differently, the LECs $D$ and $E$ are not saturated by the $\Delta$-isobar. Surprisingly, one finds that there are also no $\Delta$-contributions to the $3 \mathrm{NF}$ at $\mathrm{N}^{2} \mathrm{LO}(320)$. The $2 \pi$-exchange diagrams with one insertion of the subleading $\pi N \Delta$ vertex $\propto b_{3}+b_{8}$ generate $1 / m$-suppressed terms due to the time derivative entering this vertex. Despite the fact that both the $\Delta$-full and $\Delta$-less theories yield the same expressions for the $2 \pi$ exchange $3 \mathrm{NF}$ at $\mathrm{N}^{2} \mathrm{LO}$, one should keep in mind that the strengths of various terms are different. The extrapolation of the $\pi N$ amplitude from threshold, where the LECs are determined, to the kinematical region relevant for the $3 \mathrm{NF}$ is discussed by Pandharipande et al. (323) who claimed that both theories might yield sizeably different results if the expansion is truncated at low orders. Using the values of the LECs from Eqs. (2.43) and (2.44) one, however, finds that the strengths of various terms differ from each other at most by $\sim 7 \%$ at $\mathrm{N}^{2} \mathrm{LO}(320)$. To conclude, the only effect of including the $\Delta$-isobar as
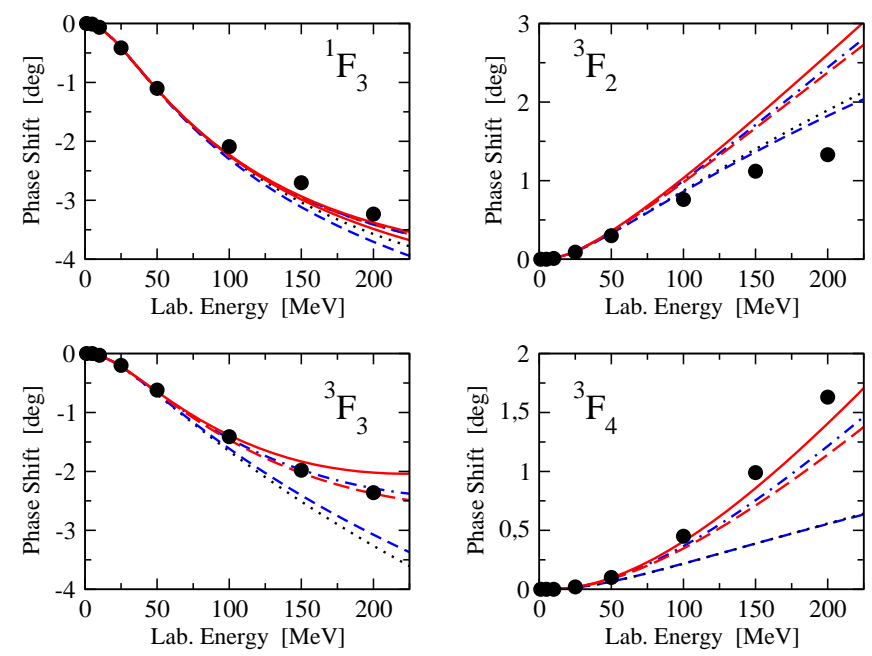

FIG. 23 F-wave NN phase shifts for $\tilde{\Lambda}=700 \mathrm{MeV}$. The dotted curve is the LO prediction, long-dashed (short-dashed) and solid (dashed-dotted) lines show the NLO and $\mathrm{N}^{2} \mathrm{LO}$ results with (without) the explicit $\Delta$-contributions. The filled circles depict the results from the Nijmegen PWA (273).

an explicit degree of freedom in the $3 \mathrm{NF}$ up to $\mathrm{N}^{2} \mathrm{LO}$ is the shift of the majour part of the $2 \pi$-exchange contribution in Eq. (2.22) from $\mathrm{N}^{2} \mathrm{LO}$ to NLO and some minor changes in the strengths of various terms in this expression.

We now discuss the role of the $\Delta$ for IB nuclear forces. The observed unnatural convergence pattern for the CSB $2 \pi$-exchange $2 \mathrm{NF}$ and CIB $3 \mathrm{NF}$ in the $\Delta$-less theory, see the discussion in section II.B, is very similar to the one for isospin-conserving $2 \pi$-exchange $2 \mathrm{NF}$ (in all cases the large contributions are proportional to the LECs $c_{3,4}$ ) and provides a strong motivation to explore the role of the $\Delta$ isobar in this case.

The leading IB $\Delta$-contributions to the $2 \pi$-exchange $2 \mathrm{NF}$ result from the corresponding triangle, box and crossed-box diagrams with one insertion of isospinbreaking pion, nucleon and delta mass shifts. The latter can be deduced from the corresponding leading strong and electromagnetic Lagrangians (320)

$$
\begin{aligned}
\mathcal{L}_{\pi \Delta, \mathrm{IB}}^{(2)}= & -\bar{T}_{i}^{\mu} c_{5}^{\Delta}\left(\chi_{+}-\left\langle\chi_{+}\right\rangle\right) \delta_{i j} g_{\mu \nu} T_{j}^{\nu} \\
\mathcal{L}_{\pi \Delta, \mathrm{IB}}^{(3)}= & -\bar{T}_{i}^{\mu} F_{\pi}^{2}\left[f_{1}^{\Delta} \delta_{i j}\left\langle Q_{+}^{2}-Q_{-}^{2}\right\rangle+f_{2}^{\Delta} \delta_{i j}\left\langle Q_{+}\right\rangle Q_{+}\right. \\
& +f_{3}^{\Delta} \delta_{i j}\left\langle Q_{+}\right\rangle^{2}+f_{4}^{\Delta}\left\langle\tau^{i} Q_{+}\right\rangle\left\langle\tau^{j} Q_{+}\right\rangle \\
& \left.+f_{5}^{\Delta}\left\langle\tau^{i} Q_{-}\right\rangle\left\langle\tau^{j} Q_{-}\right\rangle\right] g_{\mu \nu} T_{j}^{\nu}+\ldots
\end{aligned}
$$

where $c_{5}^{\Delta}$ and $f_{i}^{\Delta}$ are the LECs and the ellipses in the last line refer to strong terms which involve at least one pion field and are irrelevant for the following discussion. The masses of the physical delta fields $\left(\Delta^{++}, \Delta^{+}, \Delta^{0}, \Delta^{-}\right)$ 
can be written as

$$
\begin{aligned}
& m_{\Delta^{+}+}=\tilde{m}_{\Delta}+\frac{\delta m_{\Delta}^{1}}{2}, m_{\Delta^{+}}=\tilde{m}_{\Delta}+\frac{\delta m_{\Delta}^{1}}{6}+\frac{\delta m_{\Delta}^{2}}{2}, \\
& m_{\Delta^{0}}=\tilde{m}_{\Delta}-\frac{\delta m_{\Delta}^{1}}{6}+\frac{\delta m_{\Delta}^{2}}{2}, m_{\Delta^{-}}=\tilde{m}_{\Delta}-\frac{\delta m_{\Delta}^{1}}{2}
\end{aligned}
$$

where $\delta m_{\Delta}^{1} / \delta m_{\Delta}^{2}$ denote the equidistant/non-equidistant splittings and the mass $\tilde{m}_{\Delta}$ contains an isospin-invariant shift $\delta m_{\Delta}$ defined as $\tilde{m}_{\Delta}=\stackrel{\circ}{m}_{\Delta}+\delta m_{\Delta}$, with $\stackrel{\circ}{m}_{\Delta}$ being the delta mass in the chiral limit. The leading strong and electromagnetic contributions to the splittings $\delta m_{\Delta}^{1,2}$ can be read off from the Lagrangians in Eq. (2.49). While both strong and electromagnetic terms contribute to the equidistant splitting $\delta m_{\Delta}^{1}$, the non-equidistant one at this order of of pure electromagnetic origin. In Ref. (320), the values for $\tilde{m}_{\Delta}$ and $\delta m_{\Delta}^{1,2}$ were determined from the most recent particle data group values for $m_{\Delta^{++}}=1230.80 \pm$ $0.30 \mathrm{MeV}$ and $m_{\Delta^{0}}=1233.45 \pm 0.35 \mathrm{MeV}$ (324) together with the average mass $m_{\Delta} \equiv\left(m_{\Delta^{++}}+m_{\Delta^{+}}+m_{\Delta^{0}}+\right.$ $\left.m_{\Delta^{-}}\right) / 4=1233 \mathrm{MeV}$ from Ref. (325) which leads to

$$
\delta m_{\Delta}^{1}=-5.3 \pm 2.0 \mathrm{MeV}, \quad \delta m_{\Delta}^{2}=-1.7 \pm 2.7 \mathrm{MeV} .
$$

If the quark model relation (326) $m_{\Delta^{+}}-m_{\Delta^{0}}=m_{p}-m_{n}$ is employed instead of using the average delta mass, the results change as follows

$$
\delta m_{\Delta}^{1}=-3.9 \mathrm{MeV}, \delta m_{\Delta}^{2}=-0.3 \pm 0.3 \mathrm{MeV} .
$$

which is consistent with Eq. (2.50). Notice that the values for $\delta m_{\Delta}^{1,2}$ are of natural size. Indeed, based on naive dimensional analysis one expects $\left|\delta m_{\Delta}^{1}\right| \sim \epsilon M_{\pi}^{2} / M_{\rho} \sim$ $8 \mathrm{MeV}$ and $\left|\delta m_{\Delta}^{2}\right| \sim e^{2} M_{\rho} /(4 \pi)^{2} \sim 0.5 \mathrm{MeV}$. For a related discussion on the delta mass splittings in chiral EFT with a particular emphasis on their quark mass dependence the reader is referred to Ref.(327).

Having determined the values for the delta mass splittings, it is a straightforward (but teddious) exercise to work out the leading $\Delta$-contributions to the IB $2 \pi$ exchange potential. Notice that the $\Delta$-contributions to the $1 \pi$-exchange and contact potentials can be taken into account by a redefinition of various LECs and will, therefore, not be discussed. The explicit expressions for the $2 \pi$-exchange contributions can be found in Ref. (328). In Fig. 24 we show the CIB and CSB central, tensor and spin-spin potentials in coordinate space at $\mathrm{N}^{3} \mathrm{~L} \emptyset$ in the $\Delta$-full theory in comparison with the $\mathrm{N}^{3} \mathrm{~L} \phi$ and $\mathrm{N}^{4} \mathrm{~L} \phi$ results in the $\Delta$-less theory. While in the $\Delta$-less theory, the leading and subleading class-II $2 \pi$-exchange potential arises entirely from the pion mass difference $\delta M_{\pi}^{2}$, see the discussion in section II.B, in the $\Delta$-full theory one also finds contributions proportional to $\delta m_{\Delta}^{2}$. Although these contributions are numerically small, they provide a clear manifestation of effects which go beyond the subleading order in the $\Delta$-less theory. Furthermore, it is evident from Fig. 24 that the large portion of the $\mathrm{N}^{4} \mathrm{~L} \varnothing \mathrm{CIB}$ $2 \pi$-exchange potential in the $\Delta$-less theory is shifted to $\mathrm{N}^{3} \mathrm{~L} \emptyset$ in the theory with explicit $\Delta$ degrees of freedom
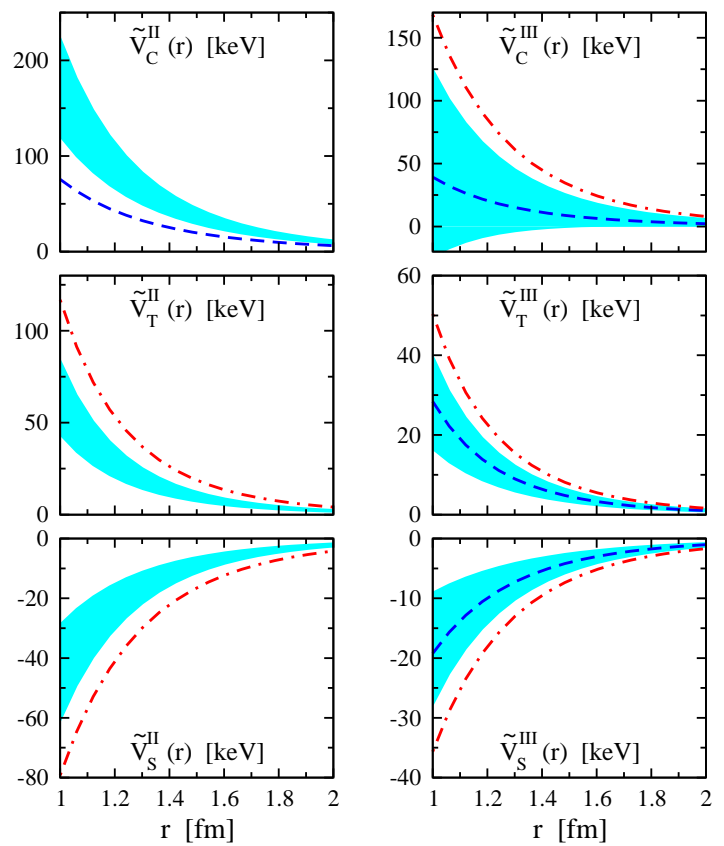

FIG. 24 Class-II (left panel) and class-III (right panel) $2 \pi$ exchange potentials at $\mathrm{N}^{3} \mathrm{~L} \emptyset$ in the $\Delta$-full theory (shaded bands) compared to the results in the $\Delta$-less theory at $\mathrm{N}^{3} \mathrm{~L} \emptyset$ (dashed lines) and $\mathrm{N}^{4} \mathrm{~L} \emptyset$ (dashed-dotted lines). The bands arise from the variation of $\delta m_{\Delta}^{1}$ and $\delta m_{\Delta}^{2}$ according to Eq. (2.50). Notice further that the leading (i.e. $\mathrm{N}^{3} \mathrm{~L} \emptyset$ ) contributions to $\tilde{V}_{T, S}^{I I}(r)$ and subleading (i.e. $\mathrm{N}^{4} \mathrm{~L} \phi$ ) contributions to $\tilde{V}_{C}^{I I}(r)$ vanish in the $\Delta$-less theory. In all cases, the spectral function cutoff $\tilde{\Lambda}=700 \mathrm{MeV}$ is used.

leading to a more natural convergence pattern. Similarly, a comparison of the corresponding CSB (i.e. class-III) potentials in two theories also indicates towards a more natural convergence in the $\Delta$-full with the main part of the unnaturally large subleading contribution in the $\Delta$-less theory being shifted to the lower order. Notice that the CSB $2 \pi$-exchange potential at $\mathrm{N}^{3} \mathrm{~L} \emptyset$ in the $\Delta$-full theory also receives contributions from the delta splitting $\delta m_{\Delta}^{1}$ which are still absent at $\mathrm{N}^{4} \mathrm{~L} \emptyset$ in the $\Delta$-less theory. For the central value, $\delta m_{\Delta}^{1}=-5.3 \mathrm{MeV}$, these contributions are numerically large and tend to cancel the ones driven by the nucleon mass difference leading to a significantly weaker resulting class-III $2 \pi$-exchange potentials as compared to the ones at subleading order in the $\Delta$-less theory. This can be viewed as an indication that certain higherorder IB contributions still missing at subleading order in the $\Delta$-less theory are unnaturally large in the theory without explicit delta degrees of freedom. Last but not least, effects from virtual $\Delta$-isobar excitation in the subleading $\pi \gamma$ - and $2 \pi$-exchange potential induced by additional one-photon exchange are considered in Ref. (223).

Inclusion of the $\Delta$ as an explicit degree of freedom has also important implications for the IB $3 \mathrm{NF}$ (320). As discussed in the previous section, the strongest IB $3 \mathrm{NF}$ arises from taking into account the charge-to-neutral pion 
mass difference in the $2 \pi$-exchange $3 \mathrm{~N}$ diagrams. In the $\Delta$-less theory, the resulting charge-symmetry conserving $3 \mathrm{NF}$, being formally subleading $\left(\mathrm{N}^{4} \mathrm{~L} \phi\right)$, is enhanced by the large values of the LECs $c_{3,4}$. In the $\Delta$-full theory, the main part of this strong $2 \pi$-exchange contribution appears already at leading order $\left(\mathrm{N}^{3} \mathrm{~L} \varnothing\right)$ indicating a more natural convergence pattern. In addition to this obvious effect, one obtains further $2 \pi$-exchange contributions at $\mathrm{N}^{3} \mathrm{~L} \emptyset$ driven by the delta and nucleon mass splittings $\delta m_{\Delta}^{2}$ (charge-symmetry conserving) and $\delta m_{\Delta}^{1}, \delta m$ (charge-symmetry breaking). A close inspection of the resulting expressions, which are all proportional to $\Delta^{-2}$, reveals that they are exactly reproduced in the $\Delta$-less theory by the saturation of the sub-subleading isospinconserving $\left(d_{i}\right.$-terms in $\left.\mathcal{L}_{\pi N}^{(2)}\right)$ and IB $\pi \pi N N$ vertices. Numerically, the strengths of these CSB 3NFs due to intermediate delta excitation turn out to be rather small, $\left|\delta m_{\Delta}^{1}-3 \delta m_{N}\right| g_{A}^{2} h_{A}^{2} M_{\pi}^{6} /\left(432 \pi^{2} F_{\pi}^{4} \Delta^{2}\right) \sim 3 \mathrm{keV}$, which, however, is comparable to the typical size of the remaining leading CSB $3 \mathrm{NF}(211), g_{A}^{4} M_{\pi}^{4} /\left(256 \pi^{2} F_{\pi}^{4}\right) \sim 7 \mathrm{keV}$.

\section{E. Few-nucleon reactions involving pions}

Few-nucleon reactions involving pions such as e.g. $\pi d \rightarrow \pi d$ (329), $\gamma d \rightarrow \pi^{0} d(330 ; 331), \pi^{3} \mathrm{He} \rightarrow$ $\pi^{3} \mathrm{He}(332), \pi^{-} d \rightarrow \gamma n n$ (333), $\gamma d \rightarrow \pi^{+} n n$ (334) and $N N \rightarrow N N \pi$ (335), where only some of the most recent references are given, provide another fascinating testing ground for the chiral EFT framework. The calculations typically utilize the distorted-wave Born approximation using transition operators derived in chiral EFT and employing either phenomenological or chiral-EFTbased wave functions for the few-nucleon states following Weinberg's original proposal (336). An important new ingredient in these applications is the appearance of the momentum scale $p=\sqrt{m M_{\pi}}$ associated with real pion production which has to be explicitly taken into account and requires an appropriate modification of the power counting (337-339). Such a modified ordering scheme was proposed in (283) and applied in (340) to calculate the pion production operator in NN collisions at threshold at NLO. Notice that the rather high energies and momenta of the nucleons in the initial state require the inclusion of the $\Delta$-isobar as an explicit degree of freedom. As a characteristic feature of the modified power counting scheme, one observes the appearance of halfinteger powers of the small parameter $\chi=M_{\pi} / \mathrm{m}$ in the expansion of the transition operators. One also finds that some pion loop contributions are promoted to significantly lower orders compared to what is expected from Weinberg's original power counting. An application of the modified power counting to $\mathrm{P}$-wave pion production in NN collisions up to $\mathrm{N}^{2} \mathrm{LO}$ is carried out in Ref. (283). At this order, only tree diagrams have to be considered. The calculations showed a satisfactory agreement with the data and also demonstrate the feasibility to extract the LEC $D$ which enters the leading 3NF, see Eq. (2.22),

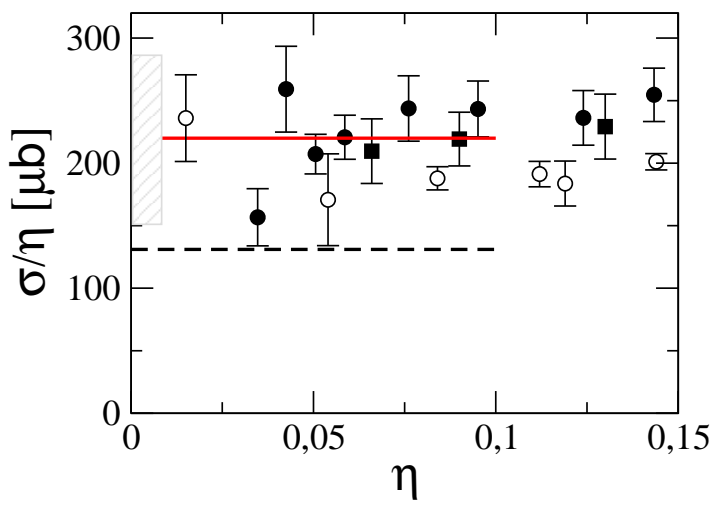

FIG. 25 LO (dashed line) and NLO (solid line) results for the total cross section for the reaction $p p \rightarrow d \pi^{+}$in comparison with the data from (342) (open circles), (343) (filled circles) and (344) (filled squares). The hatched area gives the estimated uncertainty at NLO. Figure courtesy of C. Hanhart.

from this reaction. Notice, however, that concerns have been raised in Ref. (284) regarding the convergence of the chiral expansion in this reaction. For S-wave pion production, one-loop diagrams already start to contribute at NLO. As pointed out in Ref. (341), it is important to properly separate the truly irreducible contributions in the loop diagrams from the reducible ones in order for the resulting pion production operator to be renormalizable. Numerically, the NLO loop diagrams were found to provide an important contribution to the cross section for $p p \rightarrow d \pi^{+}$. Parametrizing the near-threshold cross section for this reaction as $\sigma=\alpha \eta+\mathcal{O}\left(\eta^{3}\right)$, where $\eta$ denotes the outgoing pion momentum in units of its mass, the pion S-wave contribution at LO and NLO was found to be $\alpha^{\mathrm{LO}}=131 \mu \mathrm{b}$ and $\alpha^{\mathrm{NLO}}=220 \mu \mathrm{b}$, respectively (341). The result at NLO agrees nicely with various existing data sets, see Fig. 25. A comprehensive review of meson production reactions in nucleon-nucleon collisions can be found in Ref. (345).

Pion production reactions in few-nucleon systems also proved useful to study isospin-violating effects. Recent measurements of the forward-backward assymmetry in the process $p n \rightarrow d \pi^{0}$ (346) and the total cross section in the reaction $d d \rightarrow \alpha \pi^{0}$ (347) yielded a clear evidence of charge-symmetry breaking and serve as excellent testing ground to study isospin violation in the nuclear force and the corresponding transition operators. The first steps towards the theoretical understanding of these reactions have been taken in Refs. (348) and $(349 ; 350)$, respectively. Notice that the appearance of the four-nucleon continuum states makes the theoretical analysis of the process $d d \rightarrow \alpha \pi^{0}$ particularly challenging. We further emphasize that new data on this reaction will be provided by WASA at COSY (351). Further details on these studies and related issues can be found in a recent review article (352).

The role of the momentum scale $p=\sqrt{m M_{\pi}}$ and the related issue of the nucleon recoil effects in reactions such 
e.g. $\pi d$ scattering and pion photo- and electroproduction off the deuteron was investigated in the context of chiral EFT in Refs. (353-355). In particular, it was realized that the importance of the recoil effects in a given process is directly connected to the Pauli principle for the nucleons in the intermediate states. Notice further that the reaction $\gamma d \rightarrow n n \pi^{+}$(334) and the similar process $\pi^{-} d \rightarrow \gamma n n$ (333) were proposed as a tool to extract the value of the neutron-neutron S-wave scattering length. For more details on these and related reactions the reader is referred to Ref. (356).

\section{F. Hyperon-nucleon \& hyperon-hyperon interactions}

The effects of strange quarks in nuclear matter can e.g. be tested through the determination of the properties of so-called hyper-nuclei, in which one (or two) nucleon(s) is (are) substituted by a hyperon (hyperons). Such hyper-nuclei are produced by strangeness-exchange reactions, by associated strangeness production or by electroproduction reactions at many labs world-wide, such as CERN, BNL, KEK, DA $\Phi$ NE, JLab, MAMI and GSI, see e.g. Ref. (357). More generally, nuclear physics with strange quarks has a broad impact on contemporary physics since it lies at the intersection of nuclear and elementary particle physics. Moreover, it has significant implications to the astrophysics of compact objects. Recent progress in the field is reviewed in Ref. (358).

The hyperon-nucleon $(\mathrm{YN})$ interaction is at the heart of the hyper-nuclear binding and thus a precise determination of its various components is of utmost importance. Here, the situation is quite different compared to the two-nucleon case. The data base on YN scattering is quite poor, thus a partial wave analysis is not available and in any theoretical approach one must directly compare to data. The poor status of our information on the $\mathrm{YN}$ interaction is most clearly reflected in the present knowledge of the $\Lambda N$ scattering lengths. E.g Ref. (359) gives for the singlet $(S)$ and triplet $(T)$ scattering lenghts $a_{s}=-1.8_{-4.2}^{+2.3} \mathrm{fm}, a_{t}=-1.8_{-0.8}^{+1.1} \mathrm{fm}$, whereas in the six variants of the Nijmegen soft-core potential model $a_{s}$ varies between $-2.5 \ldots-0.7 \mathrm{fm}$ and $a_{t}$ between $-2.2 \ldots-1.8 \mathrm{fm}(360)$. In the most modern version of the Jülich meson-exchange model one finds $a_{s} \simeq-2.6 \mathrm{fm}$ and $a_{t} \simeq-1.7 \mathrm{fm}$ (361). However, for the EFT approach it is important to note that all these values are of natural size. For a proposal to extract these scattering lengths with high precision from production data, we refer to Ref. (362). Furthermore, since the masses of the $\Lambda$ and the $\Sigma$ hyperons are only about $75 \mathrm{MeV}$ apart, the coupling between the $\Lambda N$ and the $\Sigma N$ channels needs to be taken into account. Moreover, for a sensible comparison with experimental data, it is preferable to solve the scattering equation in the particle basis because then the Coulomb interaction in the charged channels can be incorporated.

The hyperon-nucleon YN interaction has not been in-
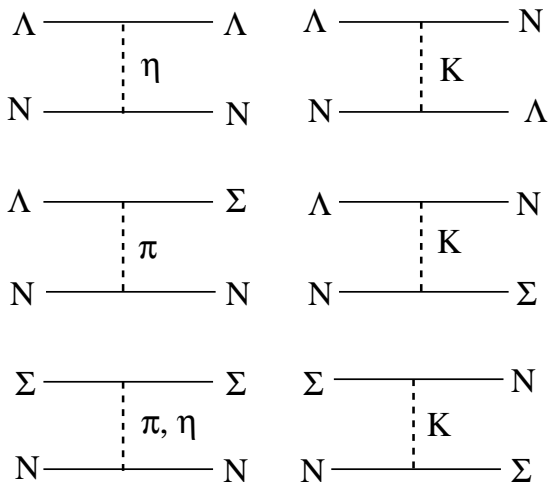

FIG. 26 One-pseudoscalar-meson-exchange diagrams at LO for the hyperon-nucleon interaction.

vestigated using EFT as extensively as the NN interaction. Hyperon and nucleon mass shifts in nuclear matter, using chiral perturbation theory, have been studied in (363). These authors used a chiral interaction containing four-baryon contact terms and pseudoscalar-meson exchanges. The hypertriton (a bound state of a proton, a neutron and a $\Lambda$ ) and $\Lambda d$ scattering were investigated in the framework of an EFT with contact interactions (364). Korpa et al. (365) performed a next-to-leading order (NLO) EFT analysis of YN scattering and hyperon mass shifts in nuclear matter. Their tree-level amplitude contains four-baryon contact terms; pseudoscalarmeson exchanges were not considered explicitly, but $\mathrm{SU}(3)$ breaking by meson masses was modeled by incorporating dimension two terms coming from one-pion exchange. The full scattering amplitude was calculated using the Kaplan-Savage-Wise resummation scheme. The hyperon-nucleon scattering data were described successfully for laboratory momenta below $200 \mathrm{MeV}$, using 12 free parameters. Some aspects of strong $\Lambda N$ scattering in effective field theory and its relation to various formulations of lattice QCD are discussed in (50).

Within the Weinberg counting scheme, a detailed investigation of the $\mathrm{YN}$ interaction at $\mathrm{LO}$ was presented in (366). At LO, the YN potential is given by onepseudoscalar-Goldstone-boson exchange diagrams, cf. Fig. 26, and contact interactons without derivatives. The spin-space part of the one-pseudoscalar-meson-exchange potential resulting from the $\mathrm{LO} \mathrm{SU}(3)$ effective chiral meson-baryon Lagrangian is,

$$
V^{B_{1} B_{2} \rightarrow B_{1}^{\prime} B_{2}^{\prime}}=-f_{B_{1} B_{1}^{\prime} P} f_{B_{2} B_{2}^{\prime} P} \frac{\vec{\sigma}_{1} \cdot \vec{k} \vec{\sigma}_{2} \cdot \vec{k}}{\vec{k}^{2}+m_{P}^{2}},
$$

where $\vec{k}$ is the momentum transfer, $P=\pi, K, \eta$, and the 


\begin{tabular}{|c|r|r|r|r|}
\hline Channel & Isospin & $\pi$ & $K$ & $\eta$ \\
\hline$N N \rightarrow N N$ & 0 & -3 & 0 & 1 \\
& 1 & 1 & 0 & 1 \\
\hline$\Lambda N \rightarrow \Lambda N$ & $\frac{1}{2}$ & 0 & 1 & 1 \\
\hline$\Lambda N \rightarrow \Sigma N$ & $\frac{1}{2}$ & $-\sqrt{3}$ & $-\sqrt{3}$ & 0 \\
\hline$\Sigma N \rightarrow \Sigma N$ & $\frac{1}{2}$ & -2 & -1 & 1 \\
& $\frac{3}{2}$ & 1 & 2 & 1 \\
\hline
\end{tabular}

TABLE I The isospin factors for the various onepseudoscalar-meson exchanges contributing to the hyperonnucleon interaction.

$f_{B_{1} B_{1}^{\prime} P}, f_{B_{2} B_{2}^{\prime} P}$ are the appropriate coupling constants

$$
\begin{aligned}
f_{N N \pi} & =f, & f_{N N \eta_{8}} & =\frac{1}{\sqrt{3}}(4 \alpha-1) f, \\
f_{\Lambda N K} & =-\frac{1}{\sqrt{3}}(1+2 \alpha) f, & f_{\Xi \Xi \pi} & =-(1-2 \alpha) f, \\
f_{\Xi \Xi \eta_{8}} & =-\frac{1}{\sqrt{3}}(1+2 \alpha) f, & f_{\Xi \Lambda K} & =\frac{1}{\sqrt{3}}(4 \alpha-1) f, \\
f_{\Lambda \Sigma \pi} & =\frac{2}{\sqrt{3}}(1-\alpha) f, & f_{\Sigma \Sigma \eta_{8}} & =\frac{2}{\sqrt{3}}(1-\alpha) f, \\
f_{\Sigma N K} & =(1-2 \alpha) f, & f_{\Sigma \Sigma \pi} & =2 \alpha f, \\
f_{\Lambda \Lambda \eta_{8}} & =-\frac{2}{\sqrt{3}}(1-\alpha) f, & f_{\Xi \Sigma K} & =-f .
\end{aligned}
$$

in terms of the coupling constant $f \equiv g_{A} / 2 F_{\pi}$ and the $F /(F+D)$-ratio $\alpha(367)$. The corresponding isospin factors for the various channels multiplying the spin-space part of the potential in Eq. (2.52) are tabulated in Tab. I. It is important to stress that while the interaction potential at LO is $\mathrm{SU}(3)$ symmetric, the kinematics of the various channels and the masses of the exchanged mesons are to be taken at their physical values. The LO contact terms for the octet baryon-baryon interactions, that are Hermitian and invariant under Lorentz transformations, are given by the $\mathrm{SU}(3)$ invariants,

$$
\begin{aligned}
& \mathcal{L}^{1}=C_{i}^{1}\left\langle\bar{B}_{a} \bar{B}_{b}\left(\Gamma_{i} B\right)_{b}\left(\Gamma_{i} B\right)_{a}\right\rangle, \\
& \mathcal{L}^{2}=C_{i}^{2}\left\langle\bar{B}_{a}\left(\Gamma_{i} B\right)_{a} \bar{B}_{b}\left(\Gamma_{i} B\right)_{b}\right\rangle, \\
& \mathcal{L}^{3}=C_{i}^{3}\left\langle\bar{B}_{a}\left(\Gamma_{i} B\right)_{a}\right\rangle\left\langle\bar{B}_{b}\left(\Gamma_{i} B\right)_{b}\right\rangle .
\end{aligned}
$$

Here, $a$ and $b$ denote the Dirac indices of the particles, $B$ is the usual irreducible octet representation of $\mathrm{SU}(3)$ given by

$$
B=\left(\begin{array}{ccc}
\frac{\Sigma^{0}}{\sqrt{2}}+\frac{\Lambda}{\sqrt{6}} & \Sigma^{+} & p \\
\Sigma^{-} & \frac{-\Sigma^{0}}{\sqrt{2}}+\frac{\Lambda}{\sqrt{6}} & n \\
-\Xi^{-} & \Xi^{0} & -\frac{2 \Lambda}{\sqrt{6}}
\end{array}\right)
$$

and the brackets denote taking the trace in the threedimensional flavor space. As an example, we display the resulting partial wave potentials for $\Lambda N \rightarrow \Lambda N$

$$
\begin{gathered}
V_{1 S 0}^{\Lambda \Lambda}=4 \pi\left[\frac{1}{6}\left(C_{S}^{1}-3 C_{T}^{1}\right)+\frac{5}{3}\left(C_{S}^{2}-3 C_{T}^{2}\right)\right. \\
\left.+2\left(C_{S}^{3}-3 C_{T}^{3}\right)\right] \\
V_{3 S 1}^{\Lambda \Lambda}=4 \pi\left[\frac{3}{2}\left(C_{S}^{1}+C_{T}^{1}\right)+\left(C_{S}^{2}+C_{T}^{2}\right)\right. \\
\left.+2\left(C_{S}^{3}+C_{T}^{3}\right)\right] .
\end{gathered}
$$

Similar expression for the isospin- $1 / 2$ and $3 / 2 \Sigma N \rightarrow \Sigma N$ and the $\Lambda N \rightarrow \Sigma N$ potentials are given in Ref. (366). Note that only 5 of the $\{8\} \times\{8\}=\{27\}+\{10\}+$ $\left\{10^{*}\right\}+\{8\}_{s}+\{8\}_{a}+\{1\}$ representations are relevant for $\mathrm{NN}$ and $\mathrm{YN}$ interactions, since the $\{1\}$ occurs only in the $\Lambda \Lambda, \Xi N$ and $\Sigma \Sigma$ channels. Equivalently, the six contact terms, $C_{S}^{1}, C_{T}^{1}, C_{S}^{2}, C_{T}^{2}, C_{S}^{3}, C_{T}^{3}$, enter the $\mathrm{NN}$ and $\mathrm{YN}$ potentials in only 5 different combinations. These 5 contact terms need to be determined by a fit to the experimental data. The resulting chiral potential $V^{\mathrm{LO}}=V_{\mathrm{OBE}}+V_{\text {cont }}$ in the LippmannSchwinger equation is regulated with a regulator function $f_{\Lambda}\left(p, p^{\prime}\right)=\exp \left(-\left(p^{4}+{p^{\prime}}^{4}\right) / \Lambda^{4}\right)$, where the cut-off $\Lambda$ is varied between 550 and $700 \mathrm{MeV}$. A fit to 35 low-energy data (total cross sections from Refs. $(359 ; 368-370)$ for $\Lambda p \rightarrow \Lambda p, \Sigma^{-} p \rightarrow \Lambda p, \Sigma^{ \pm} p \rightarrow \Sigma^{ \pm} p$ and $\Sigma^{-} p \rightarrow \Sigma^{0} n$ with hyperon lab momenta between 110 and $300 \mathrm{MeV}$ and the inelastic capture ratio at rest (371)) gives a good descripton of the data, see Fig. 27, with contact interactions of natural size. Note the strong cusp effect in $\Lambda p$ scattering at the opening of the $\Sigma^{+} n$ threshold at $p_{\text {lab }} \simeq 600 \mathrm{MeV}$ (Fig. 27b). The chiral EFT also yields a correctly bound hypertriton (372) if one fixes the relative strength of the singlet and triplet S-waves accordingly. A $\Lambda p$ singlet scattering length of $-1.9 \mathrm{fm}$ leads to the correct binding energy. The corresponding triplet scattering length is $a_{t}^{\Lambda p}=-1.2 \mathrm{fm}$ and in the $\Sigma^{+} p$ system, one finds $a_{s}=-2.3$ and $a_{t}=-0.7 \mathrm{fm}$. It is quite astonishing that with six parameters only ( 5 LECs and the cut-off $\Lambda$ ) one achieves a quite satisfatory desciption of the admittedly not very preciese YN scattering data. Clearly, a NLO calculation should be performed and fits should be done simultaneously to YN and NN data. For a more detailed discussion of these results and a comparison to more conventional approaches to the YN interaction, we refer the reader to Refs. $(366 ; 372)$.

The experimental situation on baryon-baryon scattering with $S=-2$, i.e in the YY and the $\Xi \mathrm{N}$ channels, is even poorer. Only very recently doubly strange baryon-baryon scattering data at lower energies, below $p_{\text {lab }}=0.8 \mathrm{GeV}$, were deduced for the first time $(373 ; 374)$. An upper limit of $24 \mathrm{mb}$ at $90 \%$ confidence level was provided for elastic $\Xi^{-} p$ scattering, and for the $\Xi^{-} p \rightarrow \Lambda \Lambda$ cross section at $p_{\text {lab }}=500 \mathrm{MeV}$ a value of $4.3_{-2.7}^{+6.3} \mathrm{mb}$ was reported (374). Within LO chiral EFT, baryonbaryon scattering was analyzed by Polinder et al. (375). 

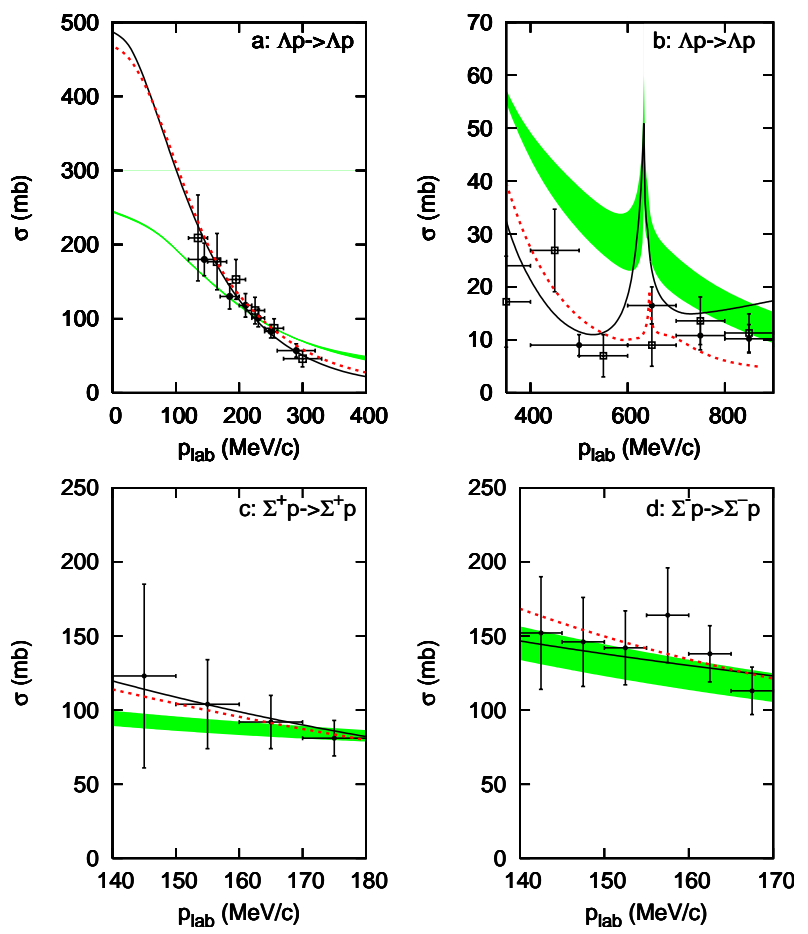

FIG. 27 Total cross sections as a function of $p_{\text {lab }}$ for $\Lambda p \rightarrow \Lambda p$ and $\Sigma^{ \pm} p \rightarrow \Sigma^{ \pm} p$. The shaded band is the LO EFT result for $\Lambda=550 \ldots 700 \mathrm{MeV}$, the dashed curve is the Jülich 04 model (361), and the solid curve is the Nijmegen NSC97f model (360). Note that the total cross sections for $\Sigma^{ \pm} p \rightarrow \Sigma^{ \pm} p$ are obtained by integrating the differential data in a limited angular range, see Ref.(369).

The contact terms and the couplings of the pseudoscalar mesons to the baryons are related via $\mathrm{SU}(3)$ symmetry to the $S=-1$ hyperon-nucleon channels. There is one additional contact interaction whose strength was varied within natural bounds in the $\Lambda \Lambda \rightarrow \Lambda \Lambda$ channel. This fixes its contribution in all other $S=-2$ baryonbaryon channels because of SU(3) symmetry. As a constraint, the information deduced from the recent candidate for ${ }_{\Lambda \Lambda}^{6} \mathrm{He}$ with a low binding energy (376), the socalled Nagara event, that suggests that the $\Lambda \Lambda$ interaction should be only moderately attractive, was imposed. For a fixed cut-off, the prediction of the $\Xi^{-} p \rightarrow \Xi^{-} p$ and the $\Xi^{-} p \rightarrow \Lambda \Lambda$ total cross section in comparison to the available data are shown in Fig. 28. The resulting $\Lambda \Lambda$ scattering length in the ${ }^{1} S_{0}$ channel is $a_{s}=$ $-1.83 \ldots-1.38 \mathrm{fm}$. For comparison, in the Nijmegen ESC04 model one finds in this channel $a_{s}=-1.32 \mathrm{fm}$ (377) and in the constituent quark model of Fujiwara et al. (378) one has $a_{s}=-0.81 \mathrm{fm}$. Note also that this contact interaction does not contribute to certain channels, so that at LO one can make parameter-free predictions for $\Sigma^{+} \Sigma^{+} \rightarrow \Sigma^{+} \Sigma^{+}, \Xi^{0} p \rightarrow \Xi^{0} p$ and $\Xi^{0} p \rightarrow \Sigma^{+} \Lambda$. It is expected that in the coming years better-quality data on the fundamental $\Xi \mathrm{N}$ and $\mathrm{YY}$ interactions as well as much more information about the physics of hyper-
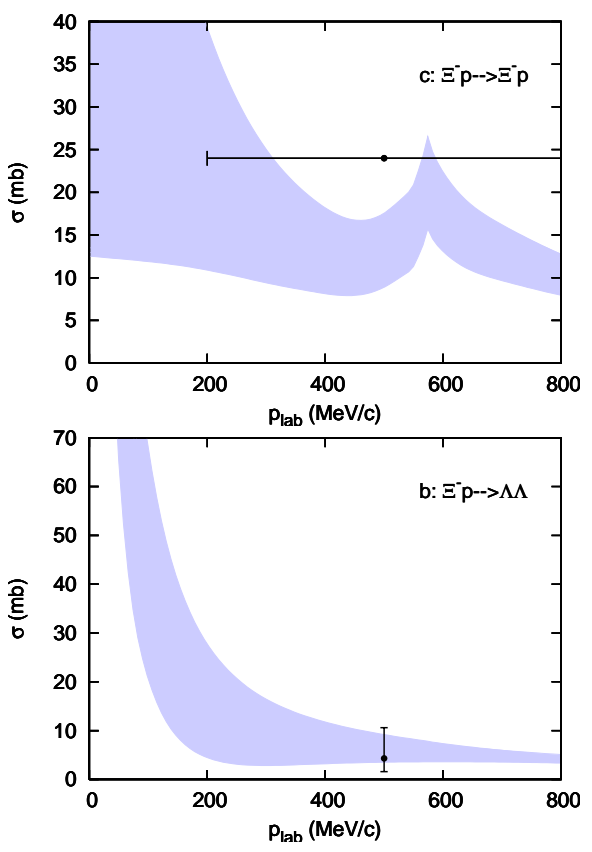

FIG. 28 Total cross sections as a function of $p_{\text {lab }}$ for $\Xi^{-} p \rightarrow$ $\Xi^{-} p$ (upper panel) and $\Xi^{-} p \rightarrow \Lambda \Lambda$. The shaded band is the LO EFT result for $\Lambda=600 \mathrm{MeV}$ and varying the LEC $C_{1 S 0}^{\Lambda \Lambda}$ of the additional singlet contact term within natural bounds gievn a mildly attractive interaction in the ${ }^{1} S_{0}$ channel of the $\Lambda \Lambda$ interaction.

nuclei will become available at the new facilities J-PARC (Japan) and FAIR (Germany). The chiral EFT developed in Ref. (375) can then be used to analyze these upcoming data in a model-independent way.

\section{G. Nuclear lattice simulations}

Once the chiral nuclear forces are determined and the low-energy constants appearing in the nuclear forces are fitted (in the two and three-nucleon sector) one can make predictions in the four- and more-nucleon sectors based on chiral EFT. However, the explicit numerical treatment of e.g. the Yakubowsky equations for more than four nucleons is a very difficult task. One possible scheme to solve the many-body problem is to put the chiral effective potential on the lattice and apply the powerful Monte-Carlo techniques which are already developed to high degree in lattice QCD. One unique feature of the lattice effective field theory approach is the ability to study in one formalism both few- and many-body systems as well as zero- and nonzero-temperature phenomena. A large portion of the nuclear phase diagram can be studied using exactly the same lattice action with exactly the same operator coefficients. A second feature is the computational advantage of many efficient Euclidean lattice methods developed for lattice QCD and condensed matter applications. This includes the use of Markov chain 
Monte Carlo techniques, Hubbard-Stratonovich transformations, and non-local updating schemes such as a hybrid Monte Carlo. A third feature is the close theoretical link between nuclear lattice simulations and chiral effective field theory. One can write down the lattice Feynman rules and calculate lattice Feynman diagrams using precisely the same action used in the non-perturbative simulation. Since the lattice formalism is based on chiral effective field theory we have a systematic power counting expansion, an a priori estimate of errors for low-energy scattering, and a clear theoretical connection to the underlying symmetries of QCD. The first studies combining lattice methods with effective field theory for low-energy nuclear physics looked at infinite nuclear and neutron matter at nonzero density and temperature (see Sec. III.E). Most of the formalism for chiral EFT on the lattice was developed in (379). Nuclear lattice simulations were used to study the triton at leading-order in pionless effective field theory with three-nucleon interactions (380).

Let us briefly discuss the principles underlying such nuclear lattice simulations (for a detailed discussion, we refer to Refs. $(379 ; 381))$. In this framework, nucleons are represented as point-like Grassman-fields and pions as point-like instantaneous pseudoscalar fields. The lattice is defined by a volume $L^{3} \times L_{t}$, with $L\left(L_{t}\right)$ the spatial (temporal) size. The corresponding lattice spacings are called $a$ and $a_{t}$, respectively. Typically, calculations are carried out using a lattice length $L \simeq 20 \mathrm{fm}$ and the lattice spacing $a \simeq 2 \mathrm{fm}$ which corresponds to the cutoff $\Lambda=\pi / a \simeq 300 \mathrm{MeV}$. At present, computational resources prevent one from using smaller lattice sizes (larger UV cutoffs). Still, the use of various forms of improved actions allows one to access the systematic errors inherent in such simulations.

The basic quantity in nuclear lattice simulations is the correlation function. For $A$ nucleons in Euclidean space it is defined by

$$
Z_{A}(t)=\left\langle\Psi_{A}|\exp (-\tau H)| \Psi_{A}\right\rangle
$$

where $\left|\Psi_{A}\right\rangle$ refers to a Slater determinants for $A$ free nucleons, $H$ is the Hamiltonian of the system and $\tau$ the Euclidean time. The ground state energy of the $A$-nucleon system can be derived from the asymptotic behavior of the correlation function for large $\tau$,

$$
E_{A}^{0}=-\lim _{\tau \rightarrow \infty} \frac{d}{d \tau} \ln Z_{A}(\tau) .
$$

The expectation value of any normal ordered operator $\mathcal{O}$ can be derived in a similar way by

$$
\begin{aligned}
\left\langle\Psi_{A}^{0}|\mathcal{O}| \Psi_{A}^{0}\right\rangle & =\lim _{\tau \leftarrow \infty} \frac{Z_{A}^{\mathcal{O}}(\tau)}{Z_{A}(\tau)}, \\
Z_{A}^{\mathcal{O}}(t) & =\left\langle\Psi_{A}|\exp (-\tau H / 2) \mathcal{O} \exp (-\tau H / 2)| \Psi_{A}\right\rangle,
\end{aligned}
$$

where $\left|\Psi_{A}^{0}\right\rangle$ denote the ground states of $A$-nucleon system. It is convenient to describe $\mathrm{NN}$ contact interactions by standard bilinear nucleon density operators using the Hubbard-Stratonovich transformation. Using the relation $\exp \left(\rho^{2} / 2\right) \sim \int d s \exp \left(-s^{2} / 2-s \rho\right)$ one can express terms quadratic in the nucleon density operator $\rho$ as terms linear in $\rho$ in the presence of auxiliary background fields (collectively denoted by $s$ ). In this representation, the full correlation function is related to the path integral over pions and auxiliary fields,

$$
\begin{aligned}
Z_{A}(t) & =\mathcal{N} \int D s \prod_{I=1,2,3} D \pi_{I} D s_{I} \exp \left(-S_{\pi \pi}-S_{s s}\right) \\
& \times\left\langle\Psi_{A}\left|M^{\left(L_{t}-1\right)}\left(\pi_{I}, s, s_{I}\right) \cdots M^{(0)}\left(\pi_{I}, s, s_{I}\right)\right| \Psi_{A}\right\rangle .
\end{aligned}
$$

Here $S_{\pi \pi}$ and $S_{s s}$ are free actions for pions and auxiliary fields $s, s_{I}$ (where $s / s_{I}$ couples to the isospinindependent/dependent nucleon bilinear), $I$ denotes isospin indices and $\mathcal{N}$ is an (irrelevant) normalization constant. $M^{(n)}$ is a transfer matrix defined as an $n$ 'th step in the temporal direction. We remark that the amplitude $\left\langle\Psi_{A}\left|M^{\left(L_{t}-1\right)}\left(\pi_{I}, s_{i}\right) \cdots M^{(0)}\left(\pi_{I}, s_{i}\right)\right| \Psi_{A}\right\rangle$ is just a Slater determinant of single nucleon matrix elements $\mathcal{M}_{i, j}$ with $i, j=1, \ldots, A$.

To be specific, we give here the leading order action starting with the free theory. The presentation here is somewhat sketchy. For an extensive discussion see (381). The free actions for the auxiliary fields and the pions are

$$
\begin{aligned}
S_{s s}\left(s, s_{I}\right) & =\frac{1}{2} \sum_{\vec{n}} s(\vec{n})^{2}+\frac{1}{2} \sum_{I=1}^{3} \sum_{\vec{n}} s_{I}(\vec{n})^{2}, \\
S_{\pi \pi}\left(\pi_{I}\right) & =\frac{\alpha_{t}}{2} \sum_{I=1}^{3} \sum_{\vec{n}} \pi_{I}(\vec{n})\left(-\Delta+M_{\pi}^{2}\right) \pi_{I}(\vec{n}),
\end{aligned}
$$

where $M_{\pi}$ is the physical pion mass and $\alpha_{t}=a_{t} / a$. For nucleons one may use an $O\left(a^{4}\right)$ improved free lattice Hamiltonian defined by

$$
\begin{gathered}
H_{\text {free }}=\frac{1}{m} \sum_{k=0}^{3} \sum_{\vec{n}_{s}, \hat{l}_{s}, i, j} f_{k}\left[a _ { i , j } ^ { \dagger } ( \vec { n } _ { s } ) \left(a_{i, j}\left(\vec{n}_{s}+k \hat{l}_{s}\right)\right.\right. \\
\left.\left.+a_{i, j}\left(\vec{n}_{s}-k \hat{l}_{s}\right)\right)\right]
\end{gathered}
$$

where the operators $a_{i, j}^{\dagger}\left(\vec{n}_{s}\right)$ and $a_{i, j}\left(\vec{n}_{s}\right)$ are the nucleon creation and annihilation operators, $\vec{n}_{s}$ are spatial coordinates, $\hat{l}_{s}$ are spatial unit vectors, the indices $i$ and $j$ represent spin and isospin indices, respectively, and the coefficients $f_{k}$ are $f_{0,1,2,3}=49 / 2,-3 / 4,3 / 40,-1 / 180$. To define the interactions one introduces nucleon-density operators with different spin/isospin polarizations

$$
\begin{aligned}
& \rho^{a^{\dagger}, a}\left(\vec{n}_{s}\right)=\sum_{i, j} a_{i, j}^{\dagger}\left(\vec{n}_{s}\right) a_{i, j}\left(\vec{n}_{s}\right) \\
& \rho_{I}^{a^{\dagger}, a}\left(\vec{n}_{s}\right)=\sum_{i, j, j^{\prime}} a_{i, j^{\prime}}^{\dagger}\left(\vec{n}_{s}\right)\left[\tau_{I}\right]_{j^{\prime}, j} a_{i, j}\left(\vec{n}_{s}\right) \\
& \rho_{I, S}^{a^{\dagger}, a}\left(\vec{n}_{s}\right)=\sum_{i, i^{\prime}, j, j^{\prime}} a_{i^{\prime}, j^{\prime}}^{\dagger}\left(\vec{n}_{s}\right)\left[\sigma_{S}\right]_{i^{\prime}, i}\left[\tau_{I}\right]_{j^{\prime}, j} a_{i, j}\left(\vec{n}_{s}\right) .
\end{aligned}
$$


The transfer matrix for $n_{t}$-th step has, besides the free part, two important contributions:

$$
\begin{aligned}
M^{\left(n_{t}\right)} & =: \exp \left\{-H_{\text {free }} \alpha_{t}\right. \\
& -\frac{g_{A} \alpha_{t}}{2 F_{\pi}} \sum_{S, I} \sum_{\vec{n}_{s}} \nabla_{S} \pi_{I}\left(\vec{n}_{s}, n_{t}\right) \rho_{S, I}^{a^{\dagger}, a}\left(\vec{n}_{s}\right) \\
& +\sqrt{-C \alpha_{t}} \sum_{\vec{n}_{s}}\left[s\left(\vec{n}_{s}, n_{t}\right) \rho^{a^{\dagger}, a}\left(\vec{n}_{s}\right)\right. \\
& \left.\left.+i \sqrt{C_{I} \alpha_{t}} \sum_{I} s_{I}\left(\vec{n}_{s}, n_{t}\right) \rho_{I}^{a^{\dagger}, a}\left(\vec{n}_{s}\right)\right]\right\}:
\end{aligned}
$$

Here :: denotes normal ordering. The first long-range contribution $\sim g_{A}$ includes the instantaneous pionnucleon interaction and describes the one-pion-exchange in the leading-order effective potential. The second shortrange contribution corresponds to the $\mathrm{NN}$ contact interactions. The low-energy constants $C=C_{S}-2 C_{T}$ and $C_{I}=-C_{T}$ (cf. sec. II.B) have different signs, $C<0, C_{I}>0$. With these signs the pion-less theory can be shown to have no sign-oscillations if the number of protons and neutrons are equal and they stay pair-wise in isospin-singlet states. In this case the multiplication with $\tau_{2}$ of the single-nucleon matrix elements $\mathcal{M}$ from left and right is well defined and gives $\tau_{2} \mathcal{M} \tau_{2}=\mathcal{M}^{*}$. For this reason, the determinant of $\mathcal{M}$ is real, $\operatorname{det} \mathcal{M}^{*}=\operatorname{det} \mathcal{M}$. Since $\tau_{2}$ is antisymmetric, the eigenvalues of $\mathcal{M}$ are doubly degenerate. This leads to a positive Slater determinant $(382 ; 383)$

$$
\operatorname{det} \mathcal{M} \geq 0
$$

The introduction of pions causes small sign oscillations which, however, are not severe and appear to be suppressed.

To perform simulations in a most efficient way one exploits the approximate SU(4)-Wigner (384) symmetry in the NN system. The symmetry transformation is given by independent rotations of the spin and isospin degrees of freedom.

$$
\delta N=\alpha_{\mu \nu} \sigma^{\mu} \tau^{\nu} N \quad \text { with } \quad \sigma^{\mu}=(1, \vec{\sigma}), \quad \tau^{\mu}=(1, \vec{\tau}) .
$$

One can show that in the limit where the NN S-wave scattering lengths approach infinity the two-nucleon system becomes invariant under the SU(4)-transformation (385). The SU(4)-breaking corrections come from the finite the scattering length and higher order terms in the chiral expansion, these are of order $\mathcal{O}\left(\left(1 / a\left({ }^{3} S_{1}\right)-\right.\right.$ $\left.\left.1 / a\left({ }^{1} S_{0}\right)\right), q / \Lambda_{\chi}\right)$. Since the NN scattering lengths are very large, the SU(4)-breaking corrections appear to be small. This fact can be used to improve the performance of the lattice simulations. The SU(4) symmetric transfer matrix is given by

$$
\begin{aligned}
M^{\left(n_{t}\right)} & =: \exp \left[-H_{\text {free }} \alpha_{t}\right. \\
& \left.+\sqrt{-C \alpha_{t}} \sum_{\vec{n}_{s}} s\left(\vec{n}_{s}, n_{t}\right) \rho^{a^{\dagger}, a}\left(\vec{n}_{s}\right)\right]: .
\end{aligned}
$$

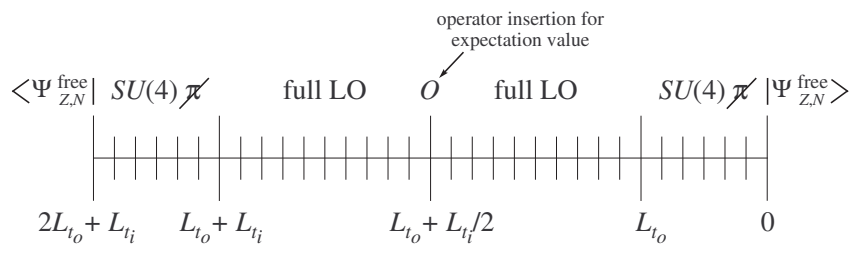

FIG. 29 Overview of the various pieces of the transfer matrix calculation.

In this case there are no sign oscillations for an even number of nucleons (386) and one only has one auxiliary field such that the simulations become much cheaper. Although there is no positivity theorem for odd numbers of nucleons, sign oscillations seem to be suppressed also in systems with odd number of nucleons because it is only one particle away from an even system with no sign oscillations. Since the final result is close to the one produced by a SU(4)-symmetric simulation it pays to divide a simulations into three parts. To simulate the expectation value of some observable one uses SU(4)-symmetric transfer matrices in the first and the last $L_{t_{0}}$ steps in order to filter the low-energy signal. After this filtering, one starts the simulation with the complete (realistic) transfer matrices. A schematic overview of the transfer matrix calculation is shown in Fig. 29. Having set up the transfer matrix, one utilizes the hybrid Monte-Carlo (HMC) method (387) to update the field configurations. More specifically, one introduces the conjugate fields $p_{\pi_{I}}$, $p_{s}, p_{s_{I}}$ and uses molecular dynamics trajectories to generate new configurations for the fields $p_{\pi_{I}}, p_{s}, p_{s_{I}}, \pi_{I}, s$, $s_{I}$ which keep the HMC Hamiltonian

$$
\begin{aligned}
H_{\mathrm{HMC}} & =\frac{1}{2} \sum_{\vec{n}}\left(\sum_{I}\left[p_{\pi_{I}}^{2}(\vec{n})+p_{s_{I}}^{2}(\vec{n})\right]+p_{s}^{2}(\vec{n})\right) \\
& +V\left(\pi_{I}, s, s_{I}\right),
\end{aligned}
$$

constant, where the HMC potential is defined by

$$
V\left(\pi_{I}, s, s_{I}\right)=S_{\pi \pi}+S_{s s}-\log |\operatorname{det} \mathcal{M}| .
$$

Upon completion of each molecular dynamics trajectory, an Metropolis accept or reject step for the new configuration according to the probability distribution $\exp \left(-H_{\mathrm{HMC}}\right)$ is applied. This process of molecular dynamics trajectory and Metropolis step is repeated many times.

Already at LO promising results for binding energies, radii and density correlations for the deuteron, triton and ${ }^{4} \mathrm{He}$ are obtained (381). On a $5^{3}$ lattice, the triton binding energy agrees with experiment within $5 \%$ and the triton root-mean-square radius is accurate to $30 \%$. The binding energy for ${ }^{4} \mathrm{He}$ is within $25 \%$ of the experimental value while the root-mean-square radius agrees within $10 \%$. Note, however, that one has to overcome a zerorange clustering instability that appears for 4 (or more 

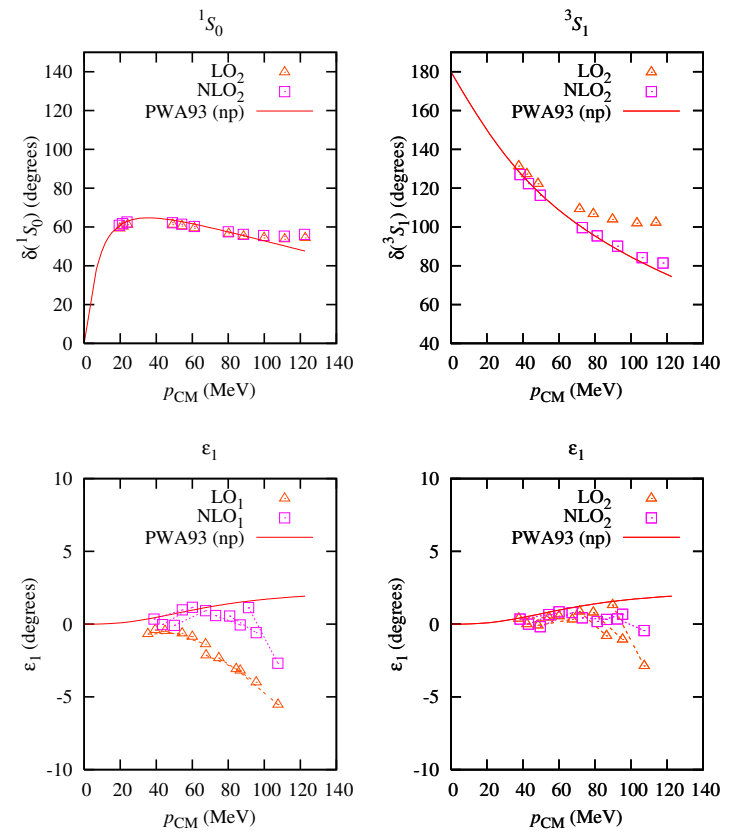

FIG. 30 NN S-wave phase shifts and mixing angles versus center-of-mass momentum with actions $\mathrm{LO}_{1}$ and $\mathrm{LO}_{2}$.

particles) which is mostly a combinatorial effect when more than two particles occupy the same lattice site (for studies of this in other systems, see (388) and references therein). To overcome this problem, one can e.g. smear out the contact interactions with a Gaussian. Such terms improve the lattice action and are formally of higher order (for more details, see e.g. Ref. (381). At LO, one can also study the feasibility of simulations for light nuclei with more than four nucleons. It was observed that for $A \leq 10$ the CPU time scales approximately linearly with the nucleon number $A$.

At NLO there appear 9 LECs which can be fitted to the Nijmegen NN scattering data and deuteron properties. Elastic scattering phase-shifts on the lattice are related by Lüscher's formula to the energy levels of twobody states in a finite large volume cubic box with periodic boundary conditions (see Sec. I.E). While this method is very useful at low momenta, it is not so useful for determining phase shifts on the lattice at higher energies and higher orbital angular momenta. Furthermore, spin-orbit coupling and partial-wave mixing are difficult to measure accurately using Lüscher's method due to multiple-scattering artifacts produced by the periodic cubic boundary conditions. In Ref. (389) a more robust approach to measure phase shifts for two nonrelativistic point particles on the lattice using a spherical wall boundary was proposed. The basic idea is to impose a hard spherical wall boundary on the relative separation between the two interacting particles at some chosen radius. The reason for this spherical wall is to remove copies of the two-particle interactions due to the periodic boundaries on the lattice. This additional boundary condition allows for a direct extraction of the phase- shifts and mixing angles from the finite-volume spectrum. For more details, see (389). Using the spherical wall method the values of 9 LECs were determined by matching three $\mathrm{S}$-wave and four $\mathrm{P}$-wave scattering data points, as well as deuteron binding energy and quadrupole moment. In Fig. 30 the NN S-wave phase-shifts and the ${ }^{3} S_{1}-{ }^{3} D_{1}$ mixing angle $\epsilon_{1}$ for two different actions, called $\mathrm{LO}_{1}$ and $\mathrm{LO}_{2}$, are displayed. The action $\mathrm{LO}_{1}$ is the one given in Eq. (2.64). In the action $\mathrm{LO}_{2}$ the contact interactions are smeared by a Gaussian. The two actions are identical at leading order and differ only by higher-order terms, thus given an estimate of the higher order corrections. As can be seen from Fig. 30, the results of the lattice simulations are in a good agreement with the partial wave results for momenta smaller than $\sim 100 \mathrm{MeV}$. Deviations between the two results for different actions appear merely at larger momenta and are consistent with the expected higher order effects. In the mean time, a novel action with spin-isopsin projected smearing has been developed that gives a good description of the partial waves up to momenta of the order of the pion mass.

At $\mathrm{N}^{2} \mathrm{LO}$ three-body forces start to show up which depend on two constants. These LECs can be determined from a fit to neutron-deuteron scattering data in the spin$1 / 2$ doublet channel and the triton binding energy. These simulations show a very natural convergence pattern with increasing chiral order. For a box length $\sim 15 \mathrm{fm}$ the volume dependence already becomes very small and the binding energy approaches its physical value. This is consistent with our expectation that the volume dependence in nuclear lattice EFT simulations should become weak for $L \sim 20 \mathrm{fm}$. In Fig. 31 the S-wave phase-shifts in the spin-3/2 quartet channel versus the square of relative momentum are shown. This channel was not taken into account in the fit procedure. Again one observes a very nice convergence with increasing chiral order. The predictions are located between the experimental data for proton-deuteron and neutron-deuteron scattering data. Since isospin-breaking was not taken into account in the simulations the results are very satisfactory. At the same order, the (Coulomb-corrected) binding energy for ${ }^{4} \mathrm{He}$ is overpredicted by $5 \%$, which is consistent with the expected theoretical accuracy of these simulations.

The results of these studies demonstrate that lattice EFT is a promising tool for a quantitative description of light nuclei. In the future, it is planned to perform $\mathrm{N}^{2} \mathrm{LO}$ Monte-Carlo simulations of light nuclei and probe neutron matter with larger number of neutrons in a box, see also Sec. III.E. In addition, more detailed studies of finite size effects and further improvements of the lattice action are called for.

\section{H. Quark mass dependence of nuclear forces and IR limit cycle in QCD}

The quark mass dependence of the chiral NN interaction was calculated to next-to-leading order (NLO) 


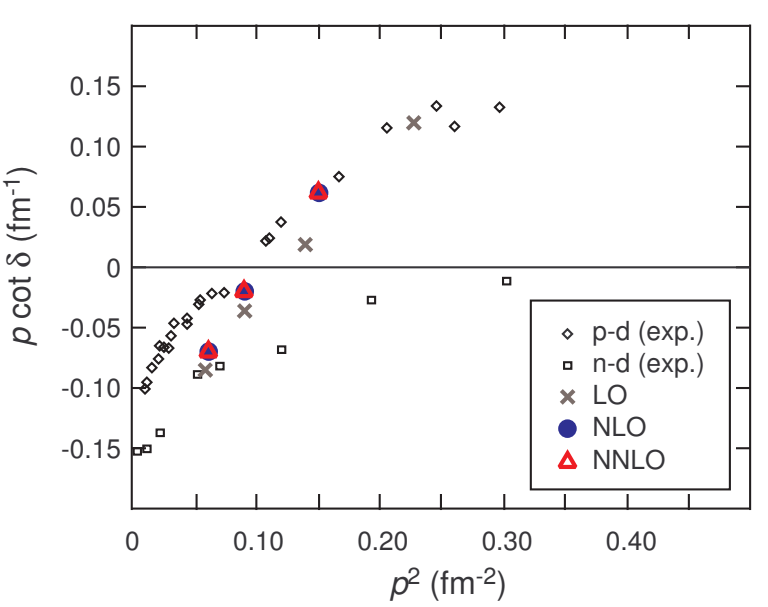

FIG. 31 Neutron-deuteron scattering S-wave phase-shifts in the spin-3/2 quartet channel versus the square of relative momentum. The data for proton-deuteron and neutron-deuteron scattering are taken from Ref. (390).

in the chiral counting in Refs. $(154 ; 391)$. At this order, the quark mass dependence is synonymous to the pion mass dependence because of the Gell-Mann-OakesRenner relation: $M_{\pi}^{2}=-\left(m_{u}+m_{d}\right)\langle 0|\bar{u} u| 0\rangle / F_{\pi}^{2}$, where $\langle 0|\bar{u} u| 0\rangle \approx(-290 \mathrm{MeV})^{3}$ is the quark condensate. In the following, we will therefore refer only to the pion mass dependence which is more convenient for nuclear applications and treat the pion mass as a parameter that can be varied by adjusting the values of the quark masses. In the work of Refs. $(154 ; 266 ; 391)$, the pion mass dependence of the nucleon-nucleon scattering lengths in the ${ }^{3} \mathrm{~S}_{1}{ }^{3} \mathrm{D}_{1}$ and ${ }^{1} \mathrm{~S}_{0}$ channels as well as the deuteron binding energy are calculated. To next-to-leading order (NLO) in the chiral power counting, the NN potential can be written as

$$
V_{\mathrm{NLO}}=V^{\mathrm{OPE}}+V^{\mathrm{TPE}}+V^{\mathrm{cont}}
$$

where $V^{\mathrm{OPE}}, V^{\mathrm{TPE}}$, and $V^{\text {cont }}$ refer to the one-pion exchange, two-pion exchange, and contact potentials, respectively. Explicit expressions can be found in Ref. (154) and section II.B.

In principle, the pion mass dependence of the chiral $\mathrm{NN}$ potential is determined uniquely. However, the extrapolation away from the physical pion mass generates errors. The dominating source are the constants $\bar{C}_{S, T}$ and $\bar{D}_{S, T}$ in $V^{\text {cont }}$ which give the corrections to the LO contact terms $\propto M_{\pi}^{2}$ and cannot be determined independently from fits to data at the physical pion mass. A smaller effect is due to the error in the LEC $\bar{d}_{16}$, which governs the pion mass dependence of $g_{A}$, from the chiral pion-nucleon Lagrangian which is enhanced as one moves away from the physical pion mass. Both effects generate increasing uncertainties as one extrapolates away from the physical point.

In the calculation of Ref. (154), the central value $\bar{d}_{16}=-1.23 \mathrm{GeV}^{-2}$ was used which is the average of

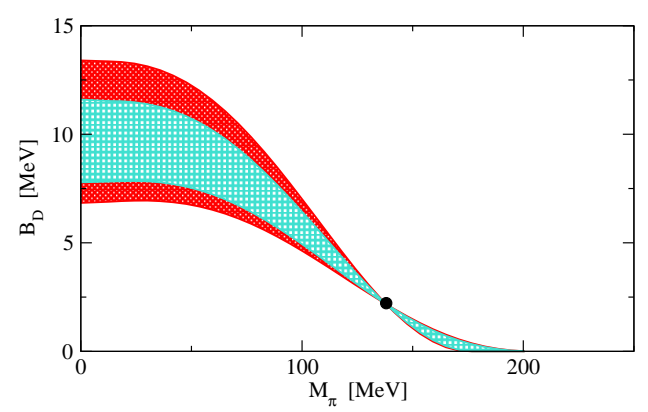

FIG. 32 Deuteron binding energy as a function of the pion mass $M_{\pi}$. The shaded areas correspond to the allowed values. The light shaded band gives the uncertainty due to the unknown value of the LECs $\bar{D}_{\mathrm{S}, \mathrm{T}}$ using the central value $\bar{d}_{16}-1.23 \mathrm{GeV}^{-2}$. The dark shaded band gives the uncertainty if, in addition to the variation of $\bar{D}_{\mathrm{S}, \mathrm{T}}$, the LEC $\bar{d}_{16}$ is varied in the range from $\bar{d}_{16}-0.91 \mathrm{GeV}^{-2}$ to $\bar{d}_{16}-1.76$ $\mathrm{GeV}^{-2}$ given in Ref. (392). The heavy dot shows the binding energy for the physical value of the pion mass.

three values given in Ref. (392). In addition, a variation of the LEC $\bar{d}_{16}$ in the range $\bar{d}_{16}=-0.91 \ldots-1.76 \mathrm{GeV}^{-2}$ as given in Ref. (392) was employed. The size of the two constants $\bar{D}_{S}$ and $\bar{D}_{T}$ was constrained from naturalness arguments. It was argued that the corresponding dimensionless constants $F_{\pi}^{2} \Lambda_{\chi}^{2} \bar{D}_{S, T}$ can be expected to satisfy the bounds:

$$
-3 \leq F_{\pi}^{2} \Lambda_{\chi}^{2} \bar{D}_{S, T} \leq 3,
$$

where $\Lambda_{\chi} \simeq 1 \mathrm{GeV}$ is the chiral symmetry breaking scale. A more conservative error estimation was given in Ref. (393). We note that Refs. (266; 391) allowed for a larger variation of these LECs. However, the bounds (2.71) are in agreement with resonance saturation estimates and similar relations are obeyed by the LECs whose values are known (280). For the constants $C_{S, T}$, e.g., this leads to $C_{S}=-120.8 \mathrm{GeV}^{-2}$ and $C_{T}=1.8 \mathrm{GeV}^{-2}$ corresponding to the dimensionless coefficients $F_{\pi}^{2} C_{S}=-1.03$ and $F_{\pi}^{2} C_{T}=0.02$, respectively. The unnaturally small value of $F_{\pi}^{2} C_{T}$ is a consequence of the approximate Wigner SU(4) symmetry.

The ranges from Eq. (2.71) were used to estimate the extrapolation errors of two-nucleon observables like the deuteron binding energy and the spin-singlet and spintriplet scattering lengths in Ref. (154). The resulting pion mass dependence of the deuteron binding energy is shown in Fig. 32. In the chiral limit the binding energy is of natural size, $B_{D} \sim F_{\pi}^{2} / m \simeq 10 \mathrm{MeV}$. Note, however, that in the calculation of $(266 ; 391)$ the assumed larger uncertainties in the LECs prevent one from making a definite statement about the binding of the deuteron in the chiral limit. For pion masses above the physical value the differences between the two calculations are considerably smaller. The recent study of Mondejar and Soto seems to indicate that two-loop diagrams generate a peculiar quark mass dependence of the contact interactions 

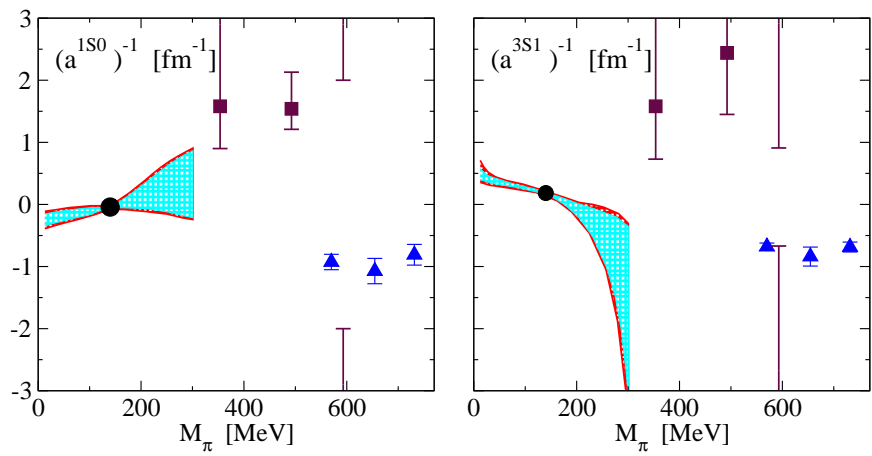

FIG. 33 Inverse of the S-wave scattering lengths in the spintriplet and spin-singlet nucleon-nucleon channels as a function of the pion mass $M_{\pi}$. Filled triangles and rectangles show the lattice calculations from Refs. $(42 ; 43)$ and (49), respectively. For remaining notation see Fig. 32.

which are parametrically large (138). The influence of these effects on the quark mass dependence of, e.g., the deuteron binding energy remains to be worked out in detail. In Fig. 33, we show the inverse scattering lengths in the spin-triplet and spin-singlet channels from Ref. (154) together with some recent lattice results (49). However, the errors and pion masses are still too large to draw any conclusions about the physical point.

Fig. 33 also shows that a scenario where both inverse scattering lengths vanish simultaneously at a critical pion mass of about $200 \mathrm{MeV}$ is possible. For pion masses below the critical value, the spin-triplet scattering length would be positive and the deuteron would be bound. As the inverse spin-triplet scattering length decreases, the deuteron would becomes more and more shallow and finally would become unbound at the critical mass. Above the critical pion mass the deuteron would exist as a shallow virtual state. In the spin-singlet channel, the situation is reversed: the "spin-singlet deuteron" would be a virtual state below the critical pion mass and would become bound above. It is unlikely that this scenario of both inverse scattering lengths vanishing simultaneously is realized in QCD at the physical values of the quark masses. However, based on this behavior it was conjectured that one should be able to reach the critical point by varying the up- and down-quark masses $m_{u}$ and $m_{d}$ independently because the spin-triplet and spin-singlet channels have different isospin (82). In this case, the triton would display the Efimov effect which corresponds to the occurence of an infrared limit cycle in QCD. It is evident that a complete investigation of this issue requires the inclusion of isospin breaking corrections and therefore higher orders in the chiral EFT. However, a number of studies have investigated the universal properties of the limit cycle by considering specific values of $\bar{D}_{S}$ and $\bar{D}_{T}$.

In the exploratory study of the three-nucleon system (82), the mean values of the error bands from (154) were used as input for the three-body calculations in the pionless EFT. Even though both scattering lengths were

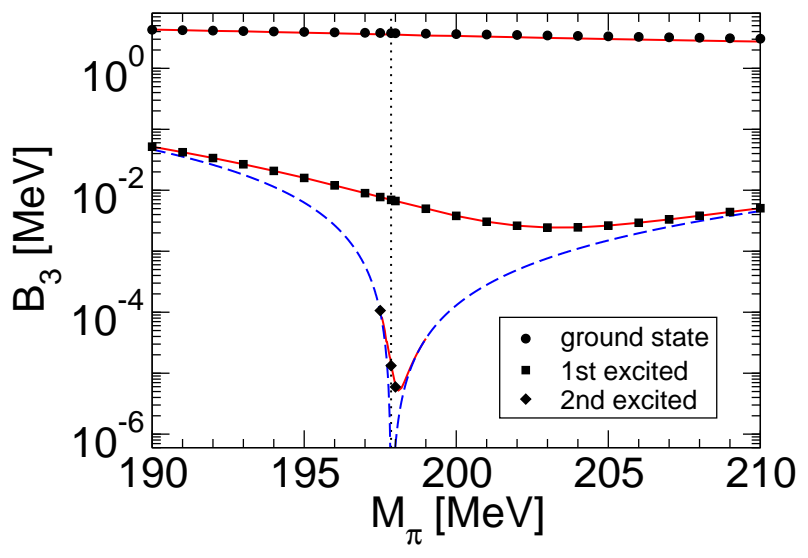

FIG. 34 Binding energies $B_{3}$ of the triton ground and first two excited states as a function of $M_{\pi}$. The circles, squares, and diamonds give the chiral EFT result, while the solid lines are calculations in the pionless theory. The vertical dotted line indicates the critical pion mass $M_{\pi}^{c r i t}$ and the dashed lines are the bound state thresholds.

large for the mean values, they did not become infinite at the same value of the pion mass and there was no exact limit cycle for this choice of parameters. However, different sets of values for $\bar{D}_{S}$ and $\bar{D}_{T}$ that lie within the bound given by Eq. (2.71) and cause the spin-singlet and spin-triplet scattering lengths to become infinite at the same value of the pion mass can be found.

In Ref. (394), the properties of the triton around the critical pion mass were studied for one particular solution with a critical pion mass $M_{\pi}^{\text {crit }}=197.8577 \mathrm{MeV}$. From the solution of the Faddeev equations, the binding energies of the triton and the first two excited states in the vicinity of the limit cycle were calculated for this scenario in chiral EFT. The binding energies are given in Fig. 34 by the circles (ground state), squares (first excited state), and diamonds (second excited state). The dashed lines indicate the neutron-deuteron $\left(M_{\pi} \leq M_{\pi}^{\text {crit }}\right)$ and neutron-spin-singlet-deuteron $\left(M_{\pi} \geq M_{\pi}^{\text {crit }}\right)$ thresholds where the three-body states become unstable. Directly at the critical mass, these thresholds coincide with the three-body threshold and the triton has infinitely many excited states. The solid lines are leading order calculations in the pionless theory using the pion mass dependence of the nucleon-nucleon scattering lengths and one triton state from chiral EFT as input. The chiral EFT results for the other triton states in the critical region are reproduced very well. The binding energy of the triton ground state varies only weakly over the whole range of pion masses and is about one half of the physical value at the critical point. The excited states are influenced by the thresholds and vary much more strongly.

These studies were extended to $\mathrm{N}^{2} \mathrm{LO}$ in the pionless EFT and neutron-deuteron scattering observables in Ref. (395). It was demonstrated that the higher order corrections in the vicinity of the critical pion mass are small. This is illustrated in Fig. 35, where we show the 


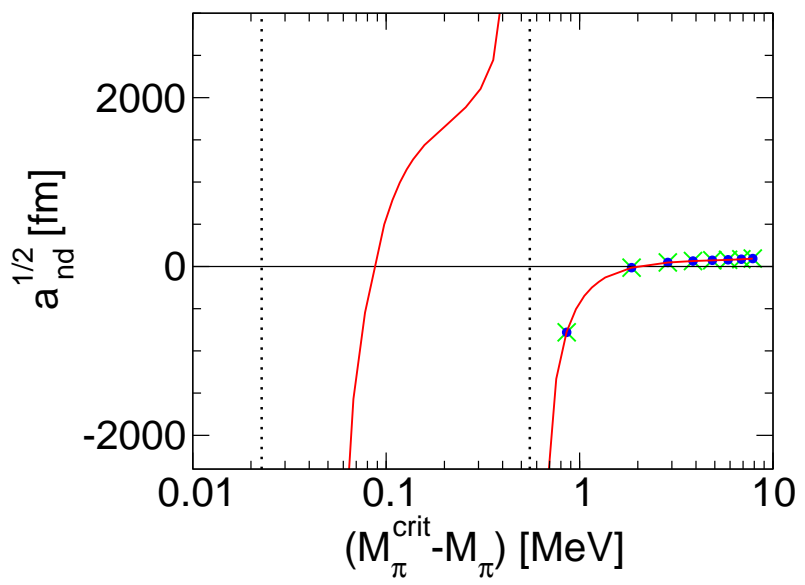

FIG. 35 Doublet neutron-deuteron scattering length $a_{n d}^{1 / 2}$ in the critical region computed in the pionless EFT. The solid line gives the LO result, while the crosses and circles show the $\mathrm{NLO}$ and $\mathrm{N}^{2} \mathrm{LO}$ results. The dotted lines indicate the pion masses at which $a_{n d}^{1 / 2}$ diverges.

doublet scattering length $a_{n d}^{1 / 2}$ in the critical region. The solid line gives the LO result, while the crosses and circles show the NLO and $\mathrm{N}^{2} \mathrm{LO}$ results. The dotted lines indicate the pion masses at which $a_{n d}^{1 / 2}$ diverges because the second and third excited states of the triton appear at the neutron-deuteron threshold. These singularities in $a_{n d}^{1 / 2}\left(M_{\pi}\right)$ are a clear signature that the limit cycle is approached in the critical region. Considering the specific case of the doublet scattering length at $M_{\pi}=190 \mathrm{MeV}$, we have

$$
a_{n d}^{1 / 2}\left(M_{\pi}=190 \mathrm{MeV}\right)=(93.18+0.80+0.14) \mathrm{fm} .
$$

At this pion mass we are fairly far away from any singular points in the function $a_{n d}^{1 / 2}\left(M_{\pi}\right)$. Consequently, the pionless results follow a natural convergence pattern with the expansion parameter $\gamma r_{0}$, which is $\approx 0.08$ at this value of $M_{\pi}$. For three-body scattering observables in the critical region no calculation in chiral EFT is available. The above example shows that both approaches complement each other. If the pion mass dependence of one threebody observable is known, the pionless theory can be used to predict all other observables with high precision and less computational effort. Figure 35 demonstrates clearly that the pionless theory converges rapidly in the critical region.

A final answer on the question of whether an infrared limit cycle can be realized in QCD can only be given by solving QCD directly. In particular, it would be very interesting to know whether this can be achieved by approriately tuning the quark masses in a Lattice QCD simulation (396). Finally, we note that the dependence of nuclear binding on hadronic mass variation has also been studied based on the Argonne potential (397).

\section{TOWARDS A MANY-BODY EFT FOR NUCLEI}

The EFT approach to the nuclear many-body problem is much less established than the one for the forces and few-nucleon systems. This is, on one hand, related to the apperance of new scales like the Fermi momentum or induced by collective excitations, and on the other hand to the computational problems related to solve the manybody problem. Thus, a variety of pathways are being explored and here we can only give a brief overview about the existing attempts and their status. For a pedagogical review on the application of EFTs to finite density systems, we refer to Furnstahl et al. (398).

\section{A. In-medium chiral perturbation theory}

Early attempts to formulate in-medium chiral perturbation theory where mostly triggered by the pioneering paper of Kaplan and Nelson on kaon condensation (399). Most of these calculations were based on chiral Lagrangians at most bilinear in the nucleon fields and performed in the mean-field approximation, $\bar{N} D N \rightarrow$ $\rho_{p} \operatorname{Tr} D_{11}+\rho_{n} \operatorname{Tr} D_{22}$ with $\rho_{p}\left(\rho_{n}\right)$ the proton (neutron) density, $D$ represents a generic differential operator including the coupling to pions and external sources, the trace runs over spinor indices and the subscripts run in flavor space. Proceeding in this way, one keeps track about the vacuum CHPT Lagrangians, but the chiral counting in the medium is lost, as e.g. nucleon correlations are not considered. The most elegant formulation of this approach based on the path-integral formulation is due to Wirzba and collaboraotrs, see e.g. (400-402).

To go beyond the mean-field approximation, the inmedium genrating functional for pions coupled to nucleons and external sources was developed in Refs. (403; 404). Leaving out multi-nucleon interactions, as systematic in-medium CHPT can be developed by expanding around the nuclear matter ground state at asymptotic times and intergrating out the nucleon fields in the path integral representation, giving rise to the in-medium generating functional (for the detailed derivation, we refer to Ref. (403))

$$
\begin{aligned}
& e^{i \tilde{\mathcal{Z}}[v, a, s, p]}=\int[d U] \exp \left\{i \int d x \mathcal{L}_{\pi \pi}-i \int \frac{d \vec{p}}{(2 \pi)^{3} 2 E(p)}\right. \\
\times & \int d x d y e^{i p(x-y)} \operatorname{Tr}\left(\left.A\left[I_{4}-D_{0}^{-1} A\right]^{-1}\right|_{(x, y)}(\not p+m) n(p)\right) \\
+ & \frac{1}{2} \int \frac{d \vec{p}}{(2 \pi)^{3} 2 E(p)} \int \frac{d \vec{q}}{(2 \pi)^{3} 2 E(q)} \int d x d x^{\prime} d y d y^{\prime} e^{i p(x-y)} \\
\times & e^{-i q\left(x^{\prime}-y^{\prime}\right)} \operatorname{Tr}\left(\left.A\left[I_{4}-D_{0}^{-1} A\right]^{-1}\right|_{\left(x, x^{\prime}\right)}(\not d+m) n(q)\right. \\
\times & \left.\left.\left.A\left[I_{4}-D_{0}^{-1} A\right]^{-1}\right|_{\left(y^{\prime}, y\right)}(\not p+m) n(p)\right)+\ldots\right\}
\end{aligned}
$$



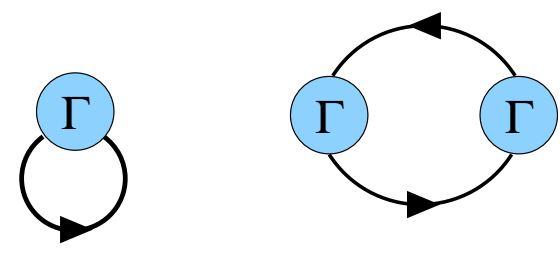

FIG. 36 Generalized in-medium vertices of lowest order. The thick solid lines correspond to insertions of a Fermi-sea and each circle to the insertion of the operator $\Gamma$ as defined in the text.

$$
\equiv \int[d U] \exp \left\{i \int d x \tilde{\mathcal{L}}_{\pi \pi}[U ; v, a, s, p]\right\},
$$

where the operator $A$ is defined as the difference between the full and the free Dirac operators,

$$
\mathcal{L}_{\bar{\psi} \psi}=\bar{\psi}(x) D(x) \psi(x)=\bar{\psi}(x)\left(D_{0}(x)-A(x)\right) \psi(x),
$$

with $D_{0}=i \gamma_{\mu} \partial^{\mu}-m$, while the diagonal flavor ma$\operatorname{trix} n(p)=\operatorname{diag}\left(\theta\left(k_{F}^{(p)}-|\vec{p}|, \theta\left(k_{F}^{(n)}-|\vec{p}|\right)\right.\right.$ parameterizes the upper cut-off of the three-momentum integrations in terms of the proton and neutron Fermi momenta, respectively. Furthermore $I_{4}$ is the unit operator in four dimension, $E(p)$ the on-shell energy of a nucleon with mass $m$ and $v, a, s, p$ are vector, axial-vector, scalar and pseudoscalar sources. The resulting in-medium effective Lagrangian $\tilde{\mathcal{L}}$ is given in terms of pions and external sources only and thus the problem is reduced to that of vacuum CHPT, with the important difference that the $\tilde{\mathcal{L}}$ is non-covariant as well as non-local (for a general analysis of the structure of non-relativistic but local EFTs see (405)). In particular, we note the appearance of the non-local vacuum vertex $\Gamma=-i A\left(I_{4}-D_{0}^{-1} A\right)^{-1}$ that generates a geometric series in terms of the local interaction operator $A$ and the free Dirac propagator, with $A$ itself being subject to the standard chiral expansion, $A=A^{(1)}+A^{(2)}+\ldots$, see (145). The generalized inmedium vertices, cf Fig. 36, consist of several non-local vacuum vertices $\Gamma$ connected through the exchange of on-shell Fermi-sea states. These are the building blocks for the systematic expansion in small momenta, counting the Fermi momentum $k_{F} \sim 2 M_{\pi}$ at nuclear saturation as $\mathcal{O}(p)$. The in-medium chiral counting including the contributions from nucleon propagators can now be given. The choice of the counting scheme depends on the energy flowing through the nucleon lines, inducing a separate consideration of the so-called standard amd nonstantard cases. Let us consider first the former. Here, the energy flow is of order $M_{\pi} \sim \mathcal{O}(p)$ and thus the nucleon propagator counts as $D_{0}^{-1} \sim \mathcal{O}\left(p^{-1}\right)$. The chiral dimension for a many-particle diagram with $L_{\pi}$ pion loops and $V_{T}$ vacuum and/or in-medium vertices of dimension $\delta_{i}$ is

$$
\nu=2 L_{\pi}+2+\sum_{j=1}^{V_{T}}\left(\delta_{j}-2\right) .
$$

Consequently, the lowest order in-medium contributions arise at $\mathcal{O}\left(p^{4}\right)$ since the lowest order in-medium vertices have dimesnion four due to the four-momentum Dirac delta-function attached to any $\Gamma$ vertex. The first corrections at NLO arise at $\mathcal{O}\left(p^{5}\right)$, which should be contraested to the vacuum case where LO (NLO) is $\mathcal{O}\left(p^{2}\right)\left(\mathcal{O}\left(p^{4}\right)\right)$. In the absence of multi-nucleon interactions, the breakdown scale is $\Lambda=\sqrt{6} \pi F_{\pi} \simeq 700 \mathrm{MeV}$ for $\mathrm{S}$-waves and $\Lambda=\sqrt{6} \pi F_{\pi} / g_{A} \simeq 560 \mathrm{MeV}$ for P-waves. However, there is one subtlety with this power counting. Quite similar to what happens in case of the TPE NN interaction, the energy flowing into a nucleon line can vanish, so that the nucleon propagator scales as $\mathcal{O}\left(p^{-2}\right)$. To deal with this non-standard case, one has to separately count the number of nucleon lines with energy $E \leq k_{F}^{2} / 2 m$ and the normal lines with $E \sim M_{\pi}$. The explicit expression for the modified counting index $\nu$ can be found in (404). In this case, the breakdown scale is $6 \pi^{2} F_{\pi}^{2} / 2 m \simeq 270 \mathrm{MeV}$ for $\mathrm{S}$-waves and further reduced by a factor of $1 / g_{A}^{2}$ for $\mathrm{P}$-wave interactions. Note that the so-defined in-medium CHPT not only encompasses but also transcends the socalled low-energy theorems of Refs. (406-408).

We now discuss some results obtained in this scheme. The density dependence of the light quark condendates is given at NLO by

$$
\begin{aligned}
& \langle\Omega|\bar{u} u| \Omega\rangle=\langle\bar{u} u\rangle_{\mathrm{vac}}\left[1-\frac{2 \sigma}{F_{\pi}^{2} M_{\pi}^{2}} \hat{\rho}+\frac{4 c_{5}}{F_{\pi}^{2}} \bar{\rho}\right], \\
& \langle\Omega|\bar{d} d| \Omega\rangle=\langle\bar{d} d\rangle_{\mathrm{vac}}\left[1-\frac{2 \sigma}{F_{\pi}^{2} M_{\pi}^{2}} \hat{\rho}-\frac{4 c_{5}}{F_{\pi}^{2}} \bar{\rho}\right],
\end{aligned}
$$

with $|\Omega\rangle$ the nuclear matter background, $\hat{\rho}=\left(\rho_{p}+\rho_{n}\right) / 2$, $\bar{\rho}=\left(\rho_{p}-\rho_{n}\right) / 2$ are the isospin symmetric and asymmetric combinations of the proton and the neutron densities, while $\sigma$ is the pion-nucleon sigma-term, $\sigma=-4 c_{1} M_{\pi}^{2}$ at $\mathcal{O}\left(p^{2}\right)$. The small isospin-breaking contribution is given in terms of the LEC $c_{5}=-(0.09 \pm 0.01) \mathrm{GeV}$. Furthermore, the subscript vac refers to the vacuum value of the corresponding quantity. Higher order corrections in the density will be discussed in the next subsection.

The propagation of pions in the medium can be analyzed by calculating the spectral relations between the energy $\omega$ and the three-momentum $\vec{q}$ for on-shell neutral and charged pions. For symmetric nuclear matter (with density $\hat{\rho}$ ) and in the chiral limit one obtains the dispersion law

$$
\omega^{2}=\vec{q}^{2}\left(1-\frac{4 \hat{\rho}}{F_{\pi}^{2}} c_{2}\right)
$$

Since the in-medium pion velocity $\tilde{v}=d \omega / d|\vec{q}|=$ $1-2 \hat{\rho} c_{2} / F_{\pi}^{2}$ must be smaller than the velcoty of light $(405 ; 409)$, this imposes the constraint $c_{2} \geq 0$, which is satisfied by the actual value of this LEC. It was also established in (404) that for standard values of the LEC $c_{3}$, chiral symmetry can account for the observed mass shift of the negatively charged pion in deeply bound pionic states in ${ }^{207} \mathrm{~Pb}(410 ; 411)$. At NLO, one obtains 
$\Delta M_{\pi}=18 \pm 5 \mathrm{MeV}$ which is compatible with the experimental result, $\Delta M_{\pi}=23 \ldots 27 \mathrm{MeV}$. Of interest is also splitting of the temporal and space-like components of the pion decay constant, which read at NLO in symmetric nuclear matter

$$
\begin{aligned}
& F_{t}=F_{\pi}\left\{1-\frac{\hat{\rho}}{\rho_{0}}(0.26 \pm 0.04)\right\}, \\
& F_{s}=F_{\pi}\left\{1-\frac{\hat{\rho}}{\rho_{0}}(1.23 \pm 0.07)\right\},
\end{aligned}
$$

with $\rho_{0}$ the nuclear matter density. The ratio $F_{s} / F_{t}=$ $\tilde{v}^{2}<1$ is consistent with the discussion about the inmedium pion velocity. One can also show that the corrections at $\mathcal{O}\left(p^{5}\right)$ do not spoil the validity of the Gell-MannOakes-Renner relation, see also (400), in particular both $F_{t}$ and the quark condensate decrease with increasing density. For a more detailed discussion of 2-, 3- and 4point functions in the medium, we reder to Ref. (404).

The missing ingedrient in these calculations are the effects of multi-nucleon interactions. It has recently been shown how these can be included in the path integral formulation $(412 ; 413)$. For that, one introduces heavy fields $H$ that couple to nucleon bilinears in appropriate spinisospin combinations, $\tilde{\mathcal{L}}[U ; v, a, s, p] \rightarrow \tilde{\mathcal{L}}[U ; H ;, a, s, p]$, and letting the mass of the $H$-fields tend to infinity, cf. Fig. 3. In that way, one can formally integrate out the multi-fermion interactions from the generating functional.

Further progress has been made by Girlanda et al. in Ref. (414). They developed a generalization of inmedium CHPT for finite systems. This provides a framework to study pion-nuclear bound states, for which the finite volume and the surface of the nucleus are important ingredients. The corresponding chiral counting is applied to the underlying pion-nucleon interactions and also to the relevant nuclear matrix elements. The central object of this approach are the Greens function in the presence of a nucleus, $G_{A}(X \rightarrow Y)$ that describe the general process $A+X \rightarrow A+Y$, where $X, Y$ represent some number of external pions and photons and $A$ is a nucleus made of $A$ nucleons. The presence of the nucleus is parameterized in terms of proton and neutron distribution functions that are taken from phenomenology. In the limit of uniform desnity, this approach reduce to the in-medium CHPT described above. As an example, the pion-nucleus optical potential is calculated at NLO,

$$
\begin{aligned}
U\left(E ; \vec{q}^{\prime}, \vec{q}\right) & =\int d^{3} \vec{x} \mathrm{e}^{-i\left(\vec{q}^{\prime}-\vec{q}\right) \cdot \vec{x}}\left[\tilde{U}\left(E ; \vec{q}^{\prime}, \vec{q}, \vec{x}\right)+\mathcal{O}\left(p^{6}\right)\right], \\
\tilde{U}\left(E ; \vec{q}^{\prime}, \vec{q}, \vec{x}\right) & =-\int d^{3} \vec{r} \frac{e^{2} E}{4 \pi|\vec{x}-\vec{r}|} 2 \rho_{p}(\vec{r})+\ldots
\end{aligned}
$$

where $E$ is the pion energy, $\vec{q}^{\prime}, \vec{q}$ are the out-going and the in-comimg three-momenta, in order, and $\rho_{p}$ is the proton charge density. The ellipsis in Eq. (3.7) stand for the contributions from the hard virtual photons and from the strong interaction, for details see (414). As particularly stressed in (415), this approach allows one to
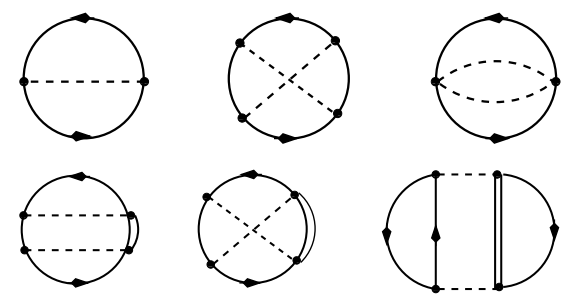

FIG. 37 Upper panel: one- and two pion exchange diagrams contributing to the energy per particle at two and three loops. Solid and dashed lines denote nucleons and pions, respectively. Lower panel: Three-body diagrams related to $2 \pi$ exchange with single delta excitations (double lines). These represent interactions between three nucleons in the Fermi sea.

identify unambiguously the nuclear finite size effects and to disentangle the S-, P - and D-wave contributions to the optical potential without invoking the local density approximation. For a more detailed discussion concerning also the comparison with more traditional approaches to pion-nuclues physics, we refer to Ref. (414).

\section{B. Perturbative chiral nuclear dynamics}

A somewhat different path, that has turned out to be very successfull phenomenologically, has been taken by the Munich group (416-418) (for a related work, see Ref. (419)). Its key element is the separation of long- and short-distance dynamics. The ordering scheme counts pion massses and momentum, the Fermi momentum and the nucleon-delta mass splitting as small quantities \#8, motivated by the fact that at nuclear matter density $k_{F} \simeq 2 M_{\pi}$. Therefore, pions must be included and propagation effects of the delta will be resolved. The longdistance physics is calculated perturbatively including one- and two pion exchange Hartree and Fock graphs, see Fig. 37 for some typical diagrams contributing to the energy per particle in nuclear matter. The short-distance dynamics is also treated perturbatively, either by finetuning of an UV cutoff or adjusting the parameters that appear at a given observable at a given order. This differs from the treatment of the contact interactions in free $\mathrm{NN}$ scattering Within this scheme, one can reproduce the empirical saturation point of nuclear matter by adjusting (fine-tuning) one parameter related to the short-distance dynamics. This corresponds to a novel mechanism for nuclear matter saturation due to the repulsive contribution to the energy per particle generated by Pauli-blocking in second order (iterated) one-pion exchange. This can be better understood by considering the realistic parame-

\footnotetext{
\#8 We unify here the two formulations with and without explicit deltas presented by this group.
} 
terization of the energy per particle in isospin symmetric nuclear matter,

$$
\bar{E}\left(k_{F}\right)=\frac{3 k_{F}^{2}}{10 m}-\alpha \frac{k_{F}^{3}}{m^{2}}+\beta \frac{k_{F}^{4}}{m^{3}},
$$

where $\alpha, \beta$ are dimensionless parameters. If $\alpha, \beta<0$, a saturation minimum will be obtained. In the deltaless theory, one obtains exactly such a form in the chiral limit and the parameters can be given in closed form. In fact, the experssion for $\beta$ is parameter-free. Fine-tuning the short-distance contribution to $\alpha$, one obtains the proper binding energy of nuclear matter. Qualitatively, this picture is not changed when the effects related to the finite pion mass and the delta-excitation are included. Furthermore, one obtains as a by-product a realistic value of the nuclear matter compressibility.

This approach has been applied and extended in varoius ways. Spin-orbit interactions in nuclei and hypernuclei were considered in (420-423). This led to a nice explanation of the very strong spin-orbit interactions in ordinary nuclei contrasted to the remarkably weak spinorbit splitting in $\Lambda$ hyper-nuclei. Corrections to the inmedium chiral condensate beyond the linear density approximation were calculated in Refs. (424; 425). Further, a systematic analysis of the nuclear energy density functional based on a unfication of chiral pion nuclear dynamics with strong scalar and vector mean fields was performed and applied to the the properties of nuclear matter and finite nuclei, see Refs. (426-429). For more details on these interesting calculations, the reader is referred to the original articles.

\section{EFT for halo nuclei}

A special class of nuclear systems exhibiting universal behavior are halo nuclei. A halo nucleus consists of a tightly bound core surrounded by one or more loosely bound valence nucleons. The valence nucleons are characterized by a very low separation energy compared to those in the core. As a consequence, the radius of the halo nucleus is large compared to the radius of the core. A trivial example is the deuteron, which can be considered a 2-body halo nucleus. The root mean square radius of the deuteron $\left\langle r^{2}\right\rangle^{1 / 2} \approx 3 \mathrm{fm}$ is about four times larger than the size of the constituent nucleons. Halo nuclei with two valence nucleons are particularly interesting examples of 3-body systems. If none of the 2-body subsystems are bound, they are called Borromean halo nuclei. This name is derived from the heraldic symbol of the Borromeo family of Italy, which consists of three rings interlocked in such way that if any one of the rings is removed the other two separate. The most carefully studied Borromean halo nuclei are ${ }^{6} \mathrm{He}$ and ${ }^{11} \mathrm{Li}$, which have two weakly bound valence neutrons $(430 ; 431)$. In the case of ${ }^{6} \mathrm{He}$, the core is a ${ }^{4} \mathrm{He}$ nucleus, which is also known as the $\alpha$ particle. The two-neutron separation energy for ${ }^{6} \mathrm{He}$ is about $1 \mathrm{MeV}$, small compared to the binding energy of the $\alpha$ particle which is about $28 \mathrm{MeV}$. The neutron- $\alpha(n \alpha)$ system has no bound states and the ${ }^{6} \mathrm{He}$ nucleus is therefore Borromean. There is, however, a strong $\mathrm{P}$-wave resonance in the $J=3 / 2$ channel of $n \alpha$ scattering which is sometimes referred to as ${ }^{5} \mathrm{He}$. This resonance is responsible for the binding of ${ }^{6} \mathrm{He}$. Thus ${ }^{6} \mathrm{He}$ can be interpreted as a bound state of an $\alpha$-particle and two neutrons, both of which are in $P_{3 / 2}$ configurations.

Because of the separation of scales in halo nuclei, they can be described by extensions of the pionless EFT. One can assume the core to be structureless and treats the nucleus as a few-body system of the core and the valence nucleons. Corrections from the structure of the core appear in higher orders and can be included in perturbation theory. Cluster models of halo nuclei then appear as leading order approximations in the EFT. A new facet is the appearance of resonances as in the neutron-alpha system which leads to a more complicated singularity structure and renormalization compared to the few-nucleon system discussed above (432).

The first application of effective field theory methods to halo nuclei was carried out in Refs. $(432 ; 433)$, where the $n \alpha$ system ("5 $\mathrm{He}$ ") was considered. It was found that for resonant $\mathrm{P}$-wave interactions both the scattering length and effective range have to be resummed at leading order. At threshold, however, only one combination of coupling constants is fine-tuned and the EFT becomes perturbative. More recent studies have focused on the consistent inclusion of the Coulomb interation in two-body halo nuclei such as the $p \alpha$ and $\alpha \alpha$ systems (434; 435). In particular, the $\alpha \alpha$ system shows a surprising amount of fine-tuning between the strong and electromagnetic interaction. It can be understood in an expansion around the limit where, when electromagnetic interactions are turned off, the ${ }^{8}$ Be ground state is exactly at threshold and exhibits conformal invariance. In this scenario, the Hoyle state in ${ }^{12} \mathrm{C}$ would appear as a remnant of an excited Efimov state (115). In order to better understand the modification of the Efimov spectrum and limit cycles by long-range interactions such as the Coulomb interaction, a one dimensional inverse square potential supplemented with a Coulomb interaction was investigated in (436). The results indicate that the counterterm required to renormalize the inverse square potential alone is sufficient to renormalize the full problem. However, the breaking of the discrete scale invariance through the Coulomb interaction leads to a modified bound state spectrum. The shallow bound states are strongly influenced by the Coulomb interaction while the deep bound states are dominated by the inverse square potential.

Three-body halo nuclei composed of a core and two valence neutrons are of particular interest due to the possibility of these systems to display the Efimov effect (83). Since the scattering length can not easily be varied in halo nuclei, one has to look for excited states. Such studies have previously been carried out in cluster models and the renormalized zero-range model (437-439). A com- 


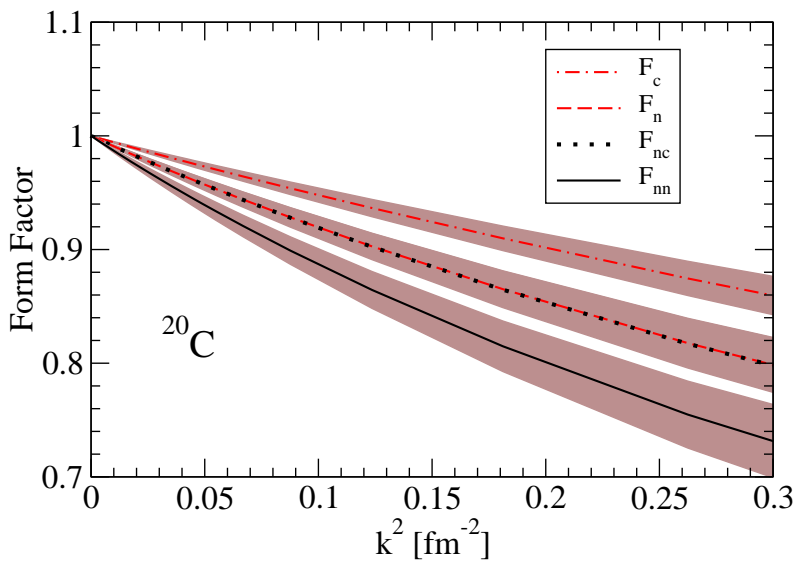

FIG. 38 The various one- and two-body matter density form factors with leading order error bands for the ground state of ${ }^{20} \mathrm{C}$ in the low-energy region: $\mathcal{F}_{n n}\left(k^{2}\right)$ black solid line; $\mathcal{F}_{n c}\left(k^{2}\right)$ black dotted line; $\mathcal{F}_{n}\left(k^{2}\right)$ lighter (red) dashed line; $\mathcal{F}_{c}\left(k^{2}\right)$ lighter (red) dot-dashed line.

prehensive study of S-wave halo nuclei in EFT including structure calculations with error estimates was recently carried out in Ref. (440). Currently, the only possible candidate for an excited Efimov state is ${ }^{20} \mathrm{C}$, which consists of a core nucleus with spin and parity quantum numbers $J^{P}=0^{+}$and two valence neutrons. The nucleus ${ }^{19} \mathrm{C}$ is expected to have a $\frac{1}{2}^{+}$state near threshold, implying a shallow neutron-core bound state and therefore a large neutron-core scattering length. The value of the ${ }^{19} \mathrm{C}$ energy, however, is not known well enough to make a definite statement about the appearance of an excited state in ${ }^{20} \mathrm{C}$. The matter form factors of halo nuclei can also be calculated in the halo EFT. As an example, we show the various one- and two-body matter density form factors with leading order error bands for the ground state of ${ }^{20} \mathrm{C}$ at low momentum transfers in Fig. 38: $\mathcal{F}_{n n}\left(k^{2}\right)$, $\mathcal{F}_{n c}\left(k^{2}\right), \mathcal{F}_{n}\left(k^{2}\right)$, and $\mathcal{F}_{c}\left(k^{2}\right)$. A definition of the form factors can be found in (440). The theory breaks down for momentum transfers of the order of the pion-mass squared $\left(k^{2} \approx 0.5 \mathrm{fm}^{-2}\right)$ where the one-pion exchange interaction cannot be approximated by short-range contact interactions anymore. From the slope of the matter form factors one can extract the corresponding radii:

$$
\mathcal{F}\left(k^{2}\right)=1-\frac{1}{6} k^{2}\left\langle r^{2}\right\rangle+\ldots
$$

Information on these radii has been extracted from experiment for some halo nuclei. For the neutron-neutron radius of the Borromean halo nucleus ${ }^{14} \mathrm{Be}$ for example, the leading order halo EFT result is $\sqrt{\left\langle r_{n n}^{2}\right\rangle}=4.1 \pm 0.5 \mathrm{fm}$. The value $\sqrt{\left\langle r_{n n}^{2}\right\rangle_{\text {exp }}}=5.4 \pm 1.0 \mathrm{fm}$ was obtained from three-body correlations in the dissociation of ${ }^{14} \mathrm{Be}$ using a technique based on intensity interferometry and Dalitz plots (441). Within the errors there is good agreement between both values. However, one should also keep in mind that there is some model dependence in the experimental result. Results for further halo nuclei are given in Ref. (440). A few recent studies have also investigated scattering observables. In particular, in Refs. (442; 443) the trajectory of the possible ${ }^{20} \mathrm{C}$ excited state was extended into the scattering region in order to find a resonance in $n-{ }^{19} \mathrm{C}$ scattering.

The simplest strange halo nucleus is the hypertriton, a 3 -body bound state of a proton, neutron, and a strange particle called the $\Lambda$. The total binding energy is only about $2.4 \mathrm{MeV}$. The hypertriton is not Borromean, because the proton-neutron subsystem has a bound state, the deuteron. The separation energy for the $\Lambda, E_{\Lambda}=$ $0.13 \pm 0.05 \mathrm{MeV}$, is tiny compared to the binding energy $B_{D}=2.224 \mathrm{MeV}$ of the deuteron. The hypertriton can therefore also be considered a 2-body halo nucleus. It has been studied in both 2-body and 3-body approaches (444-446). A study of the hypertriton in the halo EFT was carried out in Ref. (364). An important feature of the halo EFT is the possibility to quantify theoretical errors through error bands. Calculations can be improved systematically through the inclusion of higher order terms.

Another interesting application of this effective theory will be the study of Coulomb excitation data from existing and future facilities with exotic beams (such as FAIR and FRIB). In these experiments a nuclear beam scatters off the Coulomb field of a heavy nucleus. Such processes can populate excited states of the projectile which subsequently decay, leading to its "Coulomb dissociation" (447). Effective theories offer a systematic framework for a full quantum-mechanical treatment of these reactions. In summary, with new improved experimental data for these weakly bound nuclei, much more knowledge can be obtained about the structure of these interesting systems as well as discovering whether they show universal behavior and excited Efimov states.

\section{D. $V_{\text {low k }}$ potentials: construction and applications}

Nuclear interactions, like all interactions, depend on the resolution scale. For a momentum cutoff $\Lambda$, only details of the interactions at distances larger than $1 / \Lambda$ can be resolved. The interaction potential $V$ consists of $2-, 3-$, and higher-body terms and can be written as

$$
V(\Lambda)=V_{2}(\Lambda)+V_{3}(\Lambda)+V_{4}(\Lambda)+\ldots
$$

While $V$ depends on $\Lambda$, observables are independent of $\Lambda$. This property can be used to construct so-called low-momentum potentials with a cutoff $\Lambda^{\prime}<\Lambda$ that describe low-energy physics in terms of low-energy degrees of freedom only. Various methods for constructing such low-momentum potentials are known. (See, e.g. Ref. (448) for a review.) The first construction of a lowmomentum potential was carried out in $(449 ; 450)$ based on the Okubo method $(451 ; 452)$. Bogner and collaborators have pushed this idea further and constructed low-momentum potentials for various realistic nucleonnucleon interactions using renormalization group (RG) 

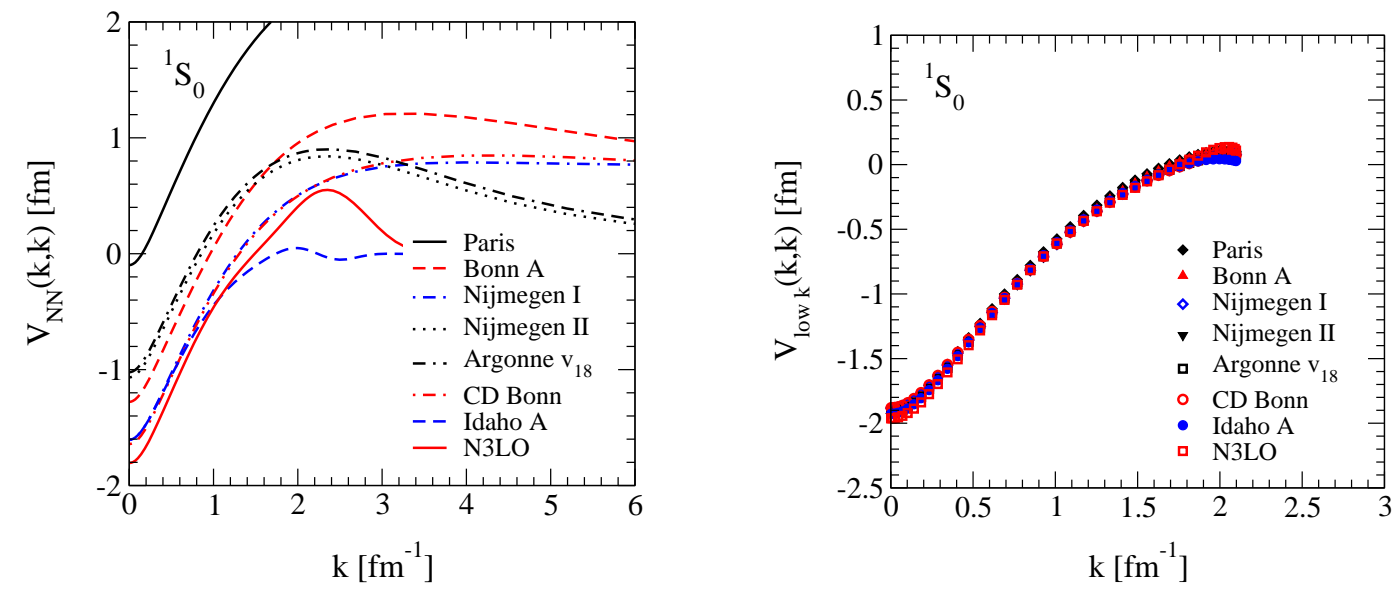

FIG. 39 The diagonal matrix elements of the nucleon-nucleon potential in the ${ }^{1} \mathrm{~S}_{0}$ partial wave. Left panel: Various realistic nucleon-nucleon potentials and the chiral $\mathrm{N}^{3} \mathrm{LO}$ potential by EM (271). Right panel: Same potentials evolved down to a cutoff $\Lambda=2.2 \mathrm{fm}^{-1}$. (Figure courtesy of A. Schwenk.)

techniques. They showed that these potentials all converge to the same universal $V_{\text {low k }}$ if the cutoff is lowered to low enough values $\left(\Lambda \lesssim 2 \mathrm{fm}^{-1}\right)$. This is illustrated in Fig. 39 for the ${ }^{1} \mathrm{~S}_{0}$ partial wave. The left panel shows various realistic nucleon-nucleon potentials and the chiral $\mathrm{N}^{3} \mathrm{LO}$ potential by EM (271). The right panel shows the same potentials evolved down to a cutoff $\Lambda=2.2 \mathrm{fm}^{-1}$. At this cutoff all potentials have collapsed to the same universal curve.

As the resolution scale is lowered the physics previously present in high-momentum modes now appears in manybody forces that are generated through the RG transformation. The low-momentum potential constructed this way is phase-equivalent by construction. In most early calculations, the many-body forces generated by the RG have been neglected for simplicity. In this case phase equivalence only holds in the 2-body subspace. Recent advances based on similarity RG techniques, however, suggest that these limitations can be overcome soon (453; 454). Low-momentum potentials have also been constructed for hyperon-nucleon interactions $(455 ; 456)$. Here the various realistic potentials are less constrained by data and the $V_{\text {low k }}$ interactions show only convergence in some channels.

In Ref. (124), the three- and four-nucleon systems were

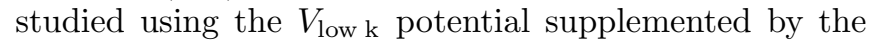
leading order chiral $3 \mathrm{~N}$ forces. This procedure was motivated by the expectation that the many body-forces have to reduce to the leading order chiral forces at low enough momentum. The free parameters in the chiral $3 \mathrm{~N}$ force were then fitted to experiment for each value of the cutoff. If the chiral $3 \mathrm{~N}$ force is left out, the cutoff variation generates the Tjon line. Since the RG evolution leaves the two-body observables unchanged by construction, the variation can only go along the Tjon line. This is in conjunction with the findings in the pionless EFT (120). For a study of potential problems with using inconsistent $2 \mathrm{~N}$ and $3 \mathrm{~N}$ low-momentum potentials in scattering calcula- tions see Ref. (457).

Because of the low cutoff, the low-momentum potential has advantages in nuclear structure calculations where smaller model spaces are desirable because of the computational effort involved. For a summary of recent applications, see Ref. (458). As the RG evolution shifts contributions between the potential and the integrals over intermediate states in loop integrals which are restricted by $\Lambda$, the RG transformation can eliminate sources of non-perturbative behavior such as strong short-range repulsion or tensor-forces $(459 ; 460)$. This suggests that perturbative nuclear matter calculations are possible. At these low resolution scales, nuclear matter saturation would be largely driven by three-body forces. Moreover, perturbative nuclear matter calculations would also provide a solid basis for the construction of a universal density functional for nuclei with controlled errors $(461 ; 462)$.

\section{E. Lattice simulations of many-nucleon systems}

Nuclear matter studies utilizing lattice simulations were pioneered by Brockmann and Frank (463), who calculated the quantum corrections to the Walecka model (464) and by Müller et al. (465), who investigated nuclear matter properties utilizing a Hamiltonian that accomodates on-site and next-to-neighbour parts of the central, spin- and isospin-exchange nucleon-nucleon interactions. The first connection between chiral EFT and the properties of nucleon and neutron matter using Monte-Carlo methods was done in the groundbreaking work of Borasoy, Lee and Schäfer (379). They laid out the framework for nuclear lattice simulations with chiral EFT and presented leading order results for hot neutron matter at temperatures $T=20-40 \mathrm{~K}$ and densities below twice the nuclear matter density. Neutron matter in a periodic box based on the lattice representation of the chiral NLO potential (see sect. II.G) was performed 


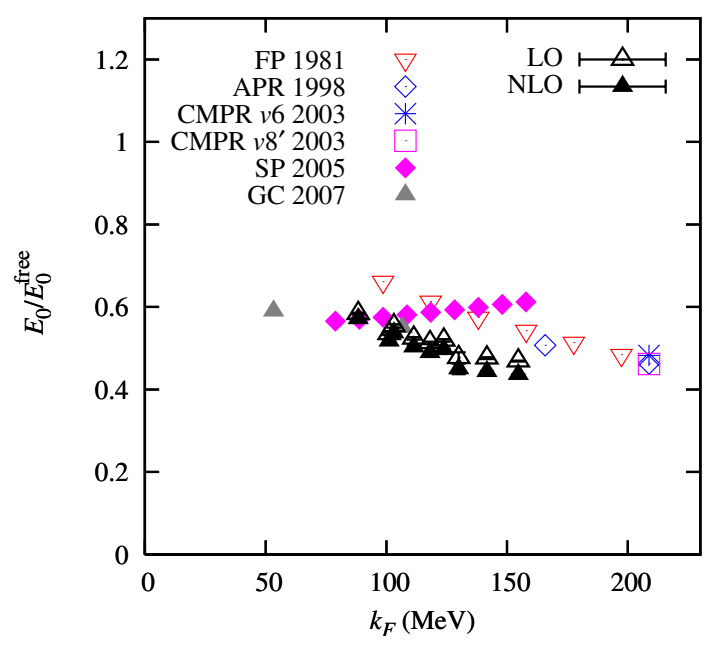

FIG. 40 Results for $E_{0} / E_{0}^{\text {free }}$ versus the Fermi momentum $k_{F}$ at LO (open triangles) and NLO (filled triangles). For comparison, we also display the results of FP 1981 (469), APR 1998 (470), CMPR $v 6$ and $v 8^{\prime} 2003$ (471), SP 2005 (472) and GC 2007 (473).

in Ref. (466), probing the density range from $2 \%$ to $8 \%$ of normal nuclear matter density. Dilute neutron matter is a particularly good testing ground for chiral EFT applied to many-nucleon systems because of the Pauli suppression of three-body forces. Furthermore, neutron matter at $k_{F} \sim 80 \mathrm{MeV}$, with $k_{F}=\left(3 \pi^{2} N\right)^{1 / 3} / L$ (for $N$ neutrons in a box of volume $L^{3}$ ), is close to the socalled unitary limit. In this limit, the scattering length is infinite and the range of the interaction is zero, so that the scattering amplitude takes its largest possible value (as given by unitarity). In this limit, the only dimensionful parameter describing the ground state of the many-fermion system is the particle density. Thus, the ground state energy $E_{0}$ of the system obeys the simple relation

$$
E_{0}=\xi E_{0}^{\mathrm{free}},
$$

where $\xi$ is a dimensionless measurable constant and $E_{0}^{\text {free }}$ the ground state energy of a free Fermi gas. Due to its universal nature, the unitary limit can be studied in ultracold atomic systems like ${ }^{6} \mathrm{Li}$ or ${ }^{40} \mathrm{Ka}$ utilizing Feshbach-resonance techniques. Recently measured values for $\xi$ scatter considerably and have sizeable error bars (for a review, see (467)). There also have been numerous calculations of $\xi$ employing very different many-body techniques, see Ref. (398) for a recent review.

Recent EFT simulations at LO and NLO with an improved action for 8, 12 and 16 neutrons boxes of length $L=10,12$ and $14 \mathrm{fm}$ are shown in Fig. 40 (468). The chiral EFT results are consistent with most earlier calculations based on different methods (note the markedly different slope obtained in (472)). A good fit to the lattice data is obtained by (the structure of the correction terms is discussed in detail in Ref. (466))

$$
\frac{E_{0}}{E_{0}^{\text {free }}} \simeq \xi-\frac{\xi_{1}}{k_{F} a}+0.16 k_{F} r_{0}-\left(0.51 \mathrm{fm}^{3}\right) k_{F}^{3},
$$

with $\xi \simeq 0.31$ and $\xi_{1} \simeq 0.81$. This is consistent with the Monte Carlo studies of many-fermion systems in Ref. (474) but smaller than the value for $\xi$ obtained in fixed-node Greens function Monte Carlo calculations, see e.g. (471). This suggests that the upper bound on the ground state energy in that type of approach might be lowered further by a more optimal fermionic nodal surface. Clearly, such numerical simulations of manynucleon systems have become a valuable tool to further constrain the nuclear equation of state at moderate densities and lead to further insight into the physics of strongly-coupled many-body systems. In particular, they provide another nice link between nuclear and atomic physics as already discussed in sec. II.A.

\section{SUMMARY AND PERSPECTIVES}

In this review, we have described the theory that has emerged by applying effective field theory methods to the nuclear force problem. This method allows for a systematic derivation of nuclear forces with a direct connection to QCD via its symmetries. The review focused on the derivation of the forces and their application in the fewnucleon problem where most work has been carried out so far. However, there are many frontiers where future work is required. These include a better understanding of nonperturbative renormalization and improved renormalization schemes, the consistent inclusion of electroweak currents, and the development of a consistent EFT for the nuclear many-body problem. The application of new techniques and advanced calculational methods for the many-body problem will be decisive to achieve the latter. Promising approaches include the renormalization group, nuclear lattice calculations, coupled cluster approaches, the no-core shell model, and density functional theory. For very low-energy processes, these approaches can be complemented by the pionless or halo EFT which is an ideal tool to unravel universal properties and establish connections to other fields of physics.

\section{Acknowledgments}

We thank all our collaborators for sharing their insights into the topics discussed here. The work of E.E. was supported in parts by funds provided from the Helmholtz Association to the young investigator group "Few-Nucleon Systems in Chiral Effective Field Theory" (grant VHNG-222). This work was further supported by the BMBF under contract No. 06BN411, by the DFG (SFB/TR 16 "Subnuclear Structure of Matter"), by the Helmholtz Association through funds provided to the virtual institute 
"Spin and strong QCD" (VH-VI-231), and by the EU Integrated Infrastructure Initiative Hadron Physics Project under contract number RII3-CT-2004-506078.

\section{References}

[1] R. D. Peccei, Lect. Notes Phys. 741, 3 (2008).

[2] C. Vafa and E. Witten, Nucl. Phys. B 234, 173 (1984).

[3] L. Giusti and S. Necco, JHEP 0704, 090 (2007).

[4] T. Banks and A. Casher, Nucl. Phys. B 169, 103 (1980).

[5] H. Leutwyler and A. Smilga, Phys. Rev. D 46 (1992) 5607.

[6] J. Stern, arXiv:hep-ph/9801282.

[7] J. Goldstone, Nuovo Cim. 19, 154 (1961).

[8] J. Goldstone, A. Salam and S. Weinberg, Phys. Rev. 127, 965 (1962).

[9] G. E. Brown, Comments Nucl. Part. Phys. 4, 140 (1970).

[10] J. L. Friar, D. G. Madland and B. W. Lynn, Phys. Rev. C 53, 3085 (1996).

[11] J. L. Friar, Few Body Syst. 22, 161 (1997).

[12] N. Kaiser, M. Muhlbauer and W. Weise, Eur. Phys. J. A 31, 53 (2007).

[13] A. Delfino, T. Frederico, V. S. Timoteo and L. Tomio, Phys. Lett. B 634, 185 (2006).

[14] H. Yukawa, Proc. Phys. Math. Soc. Jap. 17, 48 (1935).

[15] W. N. Cottingham, M. Lacombe, B. Loiseau, J. M. Richard and R. Vinh Mau, Phys. Rev. D 8, 800 (1973).

[16] A. D. Jackson, D. O. Riska and B. Verwest, Nucl. Phys. A 249, 397 (1975).

[17] R. Machleidt and I. Slaus, J. Phys. G 27, R69 (2001).

[18] R. Machleidt, Phys. Rev. C 63, 024001 (2001).

[19] V. G. J. Stoks, R. A. M. Klomp, C. P. F. Terheggen and J. J. de Swart, Phys. Rev. C 49, 2950 (1994).

[20] R. B. Wiringa, V. G. J. Stoks and R. Schiavilla, Phys. Rev. C 51, 38 (1995).

[21] S. C. Pieper and R. B. Wiringa, Ann. Rev. Nucl. Part. Sci. 51, 53 (2001).

[22] W. Glöckle, H. Witała, D. Huber, H. Kamada and J. Golak, Phys. Rept. 274, 107 (1996).

[23] J. Fujita and H. Miyazawa, Prog. Theor. Phys. 17, 360 (1957).

[24] B. H. J. McKellar and R. Rajaraman, Phys. Rev. Lett. 21, 450 (1968).

[25] S. A. Coon, M. D. Scadron and B. R. Barrett, Nucl. Phys. A 242, 467 (1975).

[26] H. T. Coelho, T. K. Das and M. R. Robilotta, Phys. Rev. C 28, 1812 (1983).

[27] B. S. Pudliner, V. R. Pandharipande, J. Carlson, S. C. Pieper and R. B. Wiringa, Phys. Rev. C 56, 1720 (1997).

[28] S. C. Pieper, V. R. Pandharipande, R. B. Wiringa and J. Carlson, Phys. Rev. C 64, 014001 (2001).

[29] H. Georgi, Ann. Rev. Nucl. Part. Sci. 43, 209 (1993).

[30] A. V. Manohar, arXiv:hep-ph/9606222.

[31] C. P. Burgess, Ann. Rev. Nucl. Part. Sci. 57, 329 (2007).

[32] K.G. Wilson, Rev. Mod. Phys. 55, 583 (1983).

[33] G.P. Lepage, "What is Renormalization?," in From Actions to Answers (TASI-89), edited by T. DeGrand and D. Toussaint (World Scientific, Singapore, 1989) [arXiv:hep-ph/0506330].
[34] S. Weinberg, Physica A 96, 327 (1979).

[35] J. Gasser and H. Leutwyler, Annals Phys. 158, 142 (1984).

[36] J. Gasser and H. Leutwyler, Nucl. Phys. B 250, 465 (1985).

[37] V. Bernard and U.-G. Meißner, Ann. Rev. Nucl. Part. Sci. 57, 33 (2007).

[38] V. Bernard, Prog. Part. Nucl. Phys. 60, 82 (2008).

[39] D. B. Kaplan, M. J. Savage and M. B. Wise, Phys. Lett. B 424, 390 (1998).

[40] D. B. Kaplan, M. J. Savage and M. B. Wise, Nucl. Phys. B 534, 329 (1998).

[41] E. Braaten and H.-W. Hammer, Phys. Rept. 428, 259 (2006).

[42] M. Fukugita, Y. Kuramashi, H. Mino, M. Okawa and A. Ukawa, Phys. Rev. Lett. 73, 2176 (1994).

[43] M. Fukugita, Y. Kuramashi, M. Okawa, H. Mino and A. Ukawa, Phys. Rev. D 52, 3003 (1995).

[44] H. W. Hamber, E. Marinari, G. Parisi and C. Rebbi, Nucl. Phys. B 225, 475 (1983).

[45] M. Lüscher, Commun. Math. Phys. 105, 153 (1986).

[46] M. Lüscher, Nucl. Phys. B 354, 531 (1991).

[47] S. R. Beane, P. F. Bedaque, A. Parreno and M. J. Savage, Phys. Lett. B 585, 106 (2004).

[48] P. F. Bedaque and U. van Kolck, Ann. Rev. Nucl. Part. Sci. 52, 339 (2002).

[49] S. R. Beane, P. F. Bedaque, K. Orginos and M. J. Savage, Phys. Rev. Lett. 97, 012001 (2006).

[50] S. R. Beane, P. F. Bedaque, A. Parreno and M. J. Savage, Nucl. Phys. A 747, 55 (2005).

[51] S. R. Beane, P. F. Bedaque, T. C. Luu, K. Orginos, E. Pallante, A. Parreno and M. J. Savage [NPLQCD Collaboration], Nucl. Phys. A 794, 62 (2007).

[52] S. R. Beane, K. Orginos and M. J. Savage, Int. J. Mod. Phys. E 17, 1157 (2008).

[53] N. Ishii, S. Aoki and T. Hatsuda, Phys. Rev. Lett. 99, 022001 (2007).

[54] S. Aoki et al. [CP-PACS Collaboration], Phys. Rev. D 71, 094504 (2005).

[55] N. Ishii, S. Aoki and T. Hatsuda, PoS LAT2007, 146 (2007).

[56] F. Wilczek, Nature 445, 156 (2007).

[57] H. Nemura, N. Ishii, S. Aoki and T. Hatsuda, arXiv:0806.1094 [nucl-th].

[58] Lattice 2008 conference website: http://conferences.jlab.org/lattice2008/ .

[59] M.K. Srivastava and D.W.L. Sprung, Adv. Nucl. Phys. 8, 121 (1975).

[60] R. Haag, Phys. Rev. 112, 669 (1958).

[61] S. Coleman, J. Wess, and B. Zumino, Phys. Rev. 177, 2239 (1969).

[62] H.D. Politzer, Nucl. Phys. B 172, 349 (1980).

[63] H. Georgi, Nucl. Phys. B 361, 339 (1991).

[64] W. Kilian and T. Ohl, Phys. Rev. D 50, 4649 (1994).

[65] S. Scherer and H.W. Fearing, Phys. Rev. D 52, 6445 (1995).

[66] C. Arzt, Phys. Lett. B 342, 189 (1995).

[67] H.W. Fearing, Phys. Rev. Lett. 81, 758 (1998).

[68] H.W. Fearing and S. Scherer, Phys. Rev. C 62, 034003 (2000).

[69] S. Scherer and H.W. Fearing, Phys. Rev. C 51, 359 (1995).

[70] J.L. Friar, D. Hüber, and U. van Kolck, Phys. Rev. C 59, 53 (1999). 
[71] T.D. Cohen, J.L. Friar, G.A. Miller, and U. van Kolck, Phys. Rev. C 53, 2661 (1996).

[72] D. B. Kaplan, M. J. Savage, and M. B. Wise, Phys. Rev. C 59, 617 (1999).

[73] I.R. Afnan and F.J.D. Serduke, Phys. Lett. 44B, 143 (1973).

[74] W.N. Polyzou and W. Glöckle, Few Body Syst. 9, 97 (1990).

[75] A. Amghar and B. Desplanques, Nucl. Phys. A 585, 657 (1995).

[76] R. J. Furnstahl, H.-W. Hammer and N. Tirfessa, Nucl. Phys. A 689, 846 (2001).

[77] H.-W. Hammer and R. J. Furnstahl, Nucl. Phys. A 678, 277 (2000).

[78] F. Coester, S. Cohen, B. Day, and C.M. Vincent, Phys. Rev. C 1, 769 (1970).

[79] R. J. Furnstahl and H.-W. Hammer, Phys. Lett. B 531, 203 (2002).

[80] M. Jaminon and C. Mahaux, Phys. Rev. C 41, 697 (1990).

[81] R. F. Mohr, R. J. Furnstahl, R. J. Perry, K. G. Wilson and H.-W. Hammer, Annals Phys. 321, 225 (2006).

[82] E. Braaten and H.-W. Hammer, Phys. Rev. Lett. 91, 102002 (2003).

[83] V. Efimov, Phy. Lett. 33B (1970) 563.

[84] E. Braaten and H.-W. Hammer, Annals Phys. 322, 120 (2007).

[85] J. Gegelia, arXiv:nucl-th/9802038.

[86] U. van Kolck, Nucl. Phys. A 645, 273 (1999).

[87] S. R. Beane, P. F. Bedaque, W. C. Haxton, D. R. Phillips and M. J. Savage, arXiv:nucl-th/0008064.

[88] S. Christlmeier and H. W. Grießhammer, Phys. Rev. C 77, 064001 (2008).

[89] N. Ryezayeva et al., Phys. Rev. Lett. 100, 172501 (2008).

[90] S. I. Ando, Eur. Phys. J. A 33, 185 (2007).

[91] D. B. Kaplan, Nucl. Phys. B 494, 471 (1997).

[92] P. F. Bedaque, H.-W. Hammer and U. van Kolck, Nucl. Phys. A 676, 357 (2000).

[93] D. R. Phillips, G. Rupak and M. J. Savage, Phys. Lett. B 473, 209 (2000).

[94] G.V. Skorniakov and K.A. Ter-Martirosian, Sov. Phys. JETP 4, 648 (1957) [J. Exptl. Theoret. Phys. (U.S.S.R.) 31, 775 (1956)].

[95] G.S. Danilov, Sov. Phys. JETP 13, 349 (1961) [J. Exptl. Theoret. Phys. (U.S.S.R.) 40, 498 (1961)].

[96] P. F. Bedaque, H.-W. Hammer and U. van Kolck, Phys. Rev. Lett. 82, 463 (1999).

[97] P. F. Bedaque, H.-W. Hammer and U. van Kolck, Nucl. Phys. A 646, 444 (1999).

[98] V.F. Kharchenko, Sov. J. Nucl. Phys. 16, 173 (1973) [Yad. Fiz. 16, 310 (1972)].

[99] H.-W. Hammer and T. Mehen, Nucl. Phys. A 690, 535 (2001).

[100] I. R. Afnan and D. R. Phillips, Phys. Rev. C 69, 034010 (2004).

[101] L. Platter, C. Ji and D. R. Phillips, arXiv:0808.1230 [cond-mat.other].

[102] P. F. Bedaque, G. Rupak, H. W. Grießhammer and H.W. Hammer, Nucl. Phys. A 714, 589 (2003).

[103] P. F. Bedaque and U. van Kolck, Phys. Lett. B 428, 221 (1998).

[104] P. F. Bedaque, H.-W. Hammer and U. van Kolck, Phys. Rev. C 58, 641 (1998).
[105] A.C. Phillips, Nucl. Phys. A 107, 209 (1968).

[106] V. Efimov, Phys. Rev. C 44, 2303 (1991).

[107] H. W. Grießhammer, Nucl. Phys. A 744, 192 (2004).

[108] L. Platter and D. R. Phillips, Few Body Syst. 40, 35 (2006).

[109] L. Platter, Phys. Rev. C 74, 037001 (2006).

[110] F. Gabbiani, P. F. Bedaque and H. W. Grießhammer, Nucl. Phys. A 675, 601 (2000).

[111] H. W. Grießhammer, Nucl. Phys. A 760, 110 (2005).

[112] T. Barford and M. C. Birse, J. Phys. A 38, 697 (2005).

[113] M. C. Birse, Phys. Rev. C 77, 047001 (2008).

[114] S. I. Ando and M. C. Birse, Phys. Rev. C 78, 024004 (2008).

[115] V. Efimov, Nucl. Phys. A 362, 45 (1981); Erratum ibid. 378, 581(E) (1982);

[116] L. Platter and H.-W. Hammer, Nucl. Phys. A 766, 132 (2006).

[117] H. Sadeghi, S. Bayegan and H. W. Grießhammer, Phys. Lett. B 643, 263 (2006).

[118] H. Sadeghi and S. Bayegan, Nucl. Phys. A 753, 291 (2005).

[119] L. Platter, H.-W. Hammer and U.-G. Meißner, Phys. Rev. A 70, 052101 (2004).

[120] L. Platter, H.-W. Hammer and U.-G. Meißner, Phys. Lett. B 607, 254 (2005).

[121] A. Nogga, H. Kamada and W. Glöckle, Phys. Rev. Lett. 85, $944(2000)$.

[122] E. Epelbaum, H. Kamada, A. Nogga, H. Witała, W. Glöckle and U.-G. Meißner, Phys. Rev. Lett. 86, 4787 (2001).

[123] E. Epelbaum, A. Nogga, W. Glöckle, H. Kamada, U.G. Meißner and H. Witała, Phys. Rev. C 66, 064001 (2002).

[124] A. Nogga, S. K. Bogner and A. Schwenk, Phys. Rev. C 70, 061002 (2004).

[125] H.-W. Hammer and L. Platter, Eur. Phys. J. A 32, 113 (2007)

[126] M.T. Yamashita, L. Tomio, A. Delfino, T. Frederico, Europhys. Lett. 75, 555 (2006).

[127] J. von Stecher, J.P. D'Incao, C.H. Greene arXiv:0810.3876.

[128] I. Stetcu, B. R. Barrett and U. van Kolck, Phys. Lett. B 653, 358 (2007).

[129] I. Stetcu, B. R. Barrett, U. van Kolck and J. P. Vary, Phys. Rev. A 76, 063613 (2007).

[130] S. Weinberg, Phys. Lett. B 251, 288 (1990).

[131] S. Weinberg, Nucl. Phys. B 363, 3 (1991).

[132] T. D. Cohen and J. M. Hansen, Phys. Rev. C 59, 13 (1999).

[133] T. D. Cohen and J. M. Hansen, Phys. Rev. C 59, 3047 (1999).

[134] S. Fleming, T. Mehen and I. W. Stewart, Nucl. Phys. A 677, 313 (2000).

[135] M. Lutz, Nucl. Phys. A 677, 241 (2000).

[136] J. A. Oller, Nucl. Phys. A 725, 85 (2003).

[137] J. Soto and J. Tarrus, Phys. Rev. C 78, 024003 (2008).

[138] J. Mondejar and J. Soto, Eur. Phys. J. A 32, 77 (2007).

[139] A. Nogga, R. G. E. Timmermans and U. van Kolck, Phys. Rev. C 72, 054006 (2005).

[140] M. C. Birse, Phys. Rev. C 74, 014003 (2006).

[141] R. J. N. Phillips, Reports on Progress in Physics XXII, 562 (1959).

[142] N. Kaiser, R. Brockmann and W. Weise, Nucl. Phys. A 625, 758 (1997). 
[143] E. Epelbaum, W. Glöckle and U.-G. Meißner, Nucl. Phys. A 637, 107 (1998).

[144] E. E. Jenkins and A. V. Manohar, Phys. Lett. B 255 (1991) 558.

[145] V. Bernard, N. Kaiser, J. Kambor and U.-G. Meißner, Nucl. Phys. B 388, 315 (1992).

[146] N. Fettes, U.-G. Meißner, M. Mojzis and S. Steininger, Annals Phys. 283, 273 (2000) [Erratum-ibid. 288, 249 (2001)].

[147] J. Gasser, M. E. Sainio and A. Svarc, Nucl. Phys. B 307, 779 (1988).

[148] E. Epelbaum, The nucleon nucleon interaction in a chiral effective field theory, $\mathrm{PhD}$ thesis, Ruhr-University Bochum, Germany, 2000, JUL-3803.

[149] C. Ordonez, L. Ray and U. van Kolck, Phys. Rev. Lett. 72, 1982 (1994).

[150] C. Ordonez, L. Ray and U. van Kolck, Phys. Rev. C 53, 2086 (1996).

[151] J. L. Friar and S. A. Coon, Phys. Rev. C 49, 1272 (1994).

[152] J. L. Friar, Annals Phys. 104, 380 (1977).

[153] E. Epelbaum, W. Glöckle and U.-G. Meißner, Nucl. Phys. A 671, 295 (2000).

[154] E. Epelbaum, U.-G. Meißner and W. Glöckle, Nucl. Phys. A 714, 535 (2003).

[155] E. Epelbaum, W. Glöckle and U.-G. Meißner, Eur. Phys. J. A 19, 125 (2004).

[156] E. Epelbaum, W. Glöckle and U.-G. Meißner, Eur. Phys. J. A 19, 401 (2004).

[157] C. Ordonez and U. van Kolck, Phys. Lett. B 291, 459 (1992).

[158] S. A. Coon and J. L. Friar, Phys. Rev. C 34, 1060 (1986).

[159] J. A. Eden and M. F. Gari, Phys. Rev. C 53, 1510 (1996).

[160] V. Bernard, N. Kaiser and U.-G. Meißner, Nucl. Phys. A 615, 483 (1997).

[161] M. R. Robilotta, Phys. Rev. C 63, 044004 (2001).

[162] T. Becher and H. Leutwyler, Eur. Phys. J. C 9, 643 (1999).

[163] E. Epelbaum, Prog. Part. Nucl. Phys. 57, 654 (2006).

[164] M. C. M. Rentmeester, R. G. E. Timmermans, J. L. Friar and J. J. de Swart, Phys. Rev. Lett. 82, 4992 (1999).

[165] M. C. M. Rentmeester, R. G. E. Timmermans and J. J. de Swart, Phys. Rev. C 67, 044001 (2003).

[166] D. R. Entem and R. Machleidt, arXiv:nucl-th/0303017.

[167] M. C. Birse and J. A. McGovern, Phys. Rev. C 70, 054002 (2004).

[168] M. C. Birse, Phys. Rev. C 76, 034002 (2007).

[169] U. van Kolck, Phys. Rev. C 49, 2932 (1994).

[170] J. L. Friar, Phys. Rev. C 60, 034002 (1999).

[171] N. Kaiser, Phys. Rev. C 64, 057001 (2001).

[172] D. R. Entem and R. Machleidt, Phys. Rev. C 66, 014002 (2002).

[173] N. Kaiser, Phys. Rev. C 65, 017001 (2002).

[174] R. Higa and M. R. Robilotta, Phys. Rev. C 68, 024004 (2003).

[175] R. Higa, M. R. Robilotta and C. A. da Rocha, Phys. Rev. C 69, 034009 (2004).

[176] R. Higa, M. R. Robilotta and C. A. da Rocha, arXiv:nucl-th/0501076.

[177] N. Kaiser, Phys. Rev. C 61 (2000) 014003.

[178] N. Kaiser, Phys. Rev. C 62 (2000) 024001.
[179] J. C. Pupin and M. R. Robilotta, Phys. Rev. C 60, 014003 (1999).

[180] N. Kaiser, Phys. Rev. C 63, 044010 (2001).

[181] V. Bernard, E. Epelbaum, H. Krebs and U.-G. Meißner, Phys. Rev. C 77, 064004 (2008).

[182] S. Ishikawa and M. R. Robilotta, Phys. Rev. C 76, 014006 (2007).

[183] V. Bernard, N. Kaiser and U.-G. Meißner, Nucl. Phys. B 457, 147 (1995).

[184] N. Fettes, U.-G. Meißner and S. Steininger, Nucl. Phys. A 640, 199 (1998).

[185] P. Buettiker and U.-G. Meißner, Nucl. Phys. A 668, 97 (2000).

[186] E. Epelbaum, Eur. Phys. J. A 34, 197 (2007).

[187] A. V. Belitsky and T. D. Cohen, Phys. Rev. C 65, 064008 (2002).

[188] T. D. Cohen and B. A. Gelman, Phys. Lett. B 540, 227 (2002).

[189] T. D. Cohen, Phys. Rev. C 66, 064003 (2002).

[190] J.-I. Fujita, M. Kawai and M. Tanifuji, Nucl. Phys. 29, 252 (1962).

[191] M. R. Robilotta, Phys. Rev. C 74, 044002 (2006) [Erratum-ibid. C 74, 059902 (2006)].

[192] E. Epelbaum, Phys. Lett. B 639, 456 (2006).

[193] H. McManus and D. O. Riska, Phys. Lett. B 92, 29 (1980).

[194] M. R. Robilotta, Phys. Rev. C 31, 974 (1985).

[195] D. Rozpedzik et al., Acta Phys. Polon. B 37, 2889 (2006).

[196] G. A. Miller, B. M. K. Nefkens and I. Slaus, Phys. Rept. 194, 1 (1990).

[197] G. A. Miller and W. T. H. Van Oers, arXiv:nuclth/9409013.

[198] C. R. Howell, et al., Phys. Lett. B 444, 252 (1998).

[199] D. E. Gonzalez Trotter et al., Phys. Rev. Lett. 83, 3788 (1999).

[200] V. Huhn et al., Phys. Rev. Lett. 85, 1190 (2000).

[201] C. R. Howell, arXiv:0805.1177 [nucl-ex].

[202] H. Leutwyler, Phys. Lett. B 378, 313 (1996).

[203] R. Urech, Nucl. Phys. B 433, 234 (1995).

[204] H. Neufeld and H. Rupertsberger, Z. Phys. C 71, 131 (1996).

[205] U.-G. Meißner, G. Müller and S. Steininger, Phys. Lett. B 406, 154 (1997) [Erratum-ibid. B 407, 454 (1997)].

[206] M. Knecht and R. Urech, Nucl. Phys. B 519, 329 (1998).

[207] U.-G. Meißner and S. Steininger, Phys. Lett. B 419, 403 (1998).

[208] G. Müller and U.-G. Meißner, Nucl. Phys. B 556, 265 (1999).

[209] J. Gasser, M. A. Ivanov, E. Lipartia, M. Mojzis and A. Rusetsky, Eur. Phys. J. C 26, 13 (2002).

[210] M. Walzl, U.-G. Meißner and E. Epelbaum, Nucl. Phys. A 693, 663 (2001).

[211] E. Epelbaum, U.-G. Meißner and J. E. Palomar, Phys. Rev. C 71, 024001 (2005).

[212] E. Epelbaum and U.-G. Meißner, Phys. Rev. C 72, 044001 (2005).

[213] U. L. Van Kolck, Soft Physics: Applications Of Effective Chiral Lagrangians To Nuclear Physics And Quark Models, PhD thesis, University of Texas, Austin, USA, 1993, UNI-94-01021.

[214] U. van Kolck, J. L. Friar and J. T. Goldman, Phys. Lett. B 371, 169 (1996).

[215] J. L. Friar and U. van Kolck, Phys. Rev. C 60, 034006 
(1999).

[216] J. L. Friar, U. van Kolck, G. L. Payne and S. A. Coon, Phys. Rev. C 68, 024003 (2003).

[217] J. L. Friar, U. van Kolck, M. C. M. Rentmeester and R. G. E. Timmermans, Phys. Rev. C 70, 044001 (2004).

[218] J. L. Friar, G. L. Payne and U. van Kolck, Phys. Rev. C 71, 024003 (2005).

[219] J. Gasser and H. Leutwyler, Phys. Rept. 87, 77 (1982).

[220] S. R. Beane, K. Orginos and M. J. Savage, Nucl. Phys. B 768, 38 (2007).

[221] E. M. Henley and G. A. Miller, In *Rho M, Wilkinson D: Mesons In Nuclei, Vol.I*, Amsterdam 1979, 405-434

[222] G. J. M. Austen and J. J. de Swart, Phys. Rev. Lett. 50, 2039 (1983).

[223] N. Kaiser, Phys. Rev. C 73, 044001 (2006).

[224] E.A. Uehling, Phys. Rev. 48, 55 (1935).

[225] L. Durand, Phys. Rev. 108, 1597 (1957).

[226] V.G. Stoks, The magnetic moment interaction in NN phase shift analysis, PhD thesis, University Nijmegen, The Netherlands, 1990.

[227] A. Kievsky, M. Viviani and L. E. Marcucci, Phys. Rev. C 69, 014002 (2004).

[228] H. Witała, J. Golak, R. Skibinski, C. R. Howell and W. Tornow, Phys. Rev. C 67, 064002 (2003).

[229] G. Rupak and X. W. Kong, Nucl. Phys. A 717, 73 (2003).

[230] J. J. de Swart, M. C. M. Rentmeester and R. G. E. Timmermans, PiN Newslett. 13, 96 (1997).

[231] C. Y. Cheung, E. M. Henley and G. A. Miller, Nucl. Phys. A 348, 365 (1980).

[232] U. van Kolck, M. C. M. Rentmeester, J. L. Friar, J. T. Goldman and J. J. de Swart, Phys. Rev. Lett. 80, 4386 (1998).

[233] S. A. Coon and J. A. Niskanen, Phys. Rev. C 53, 1154 (1996).

[234] J. A. Niskanen, Phys. Rev. C 65, 037001 (2002).

[235] U.-G. Meißner, U. Raha and A. Rusetsky, Phys. Lett. B 639, 478 (2006).

[236] N. Kaiser, Phys. Rev. C 73, 064003 (2006).

[237] N. Kaiser, Phys. Rev. C 74, 067001 (2006).

[238] S. R. Beane, T. D. Cohen and D. R. Phillips, Nucl. Phys. A 632, 445 (1998).

[239] D. R. Phillips, S. R. Beane and T. D. Cohen, Annals Phys. 263, 255 (1998).

[240] M. C. Birse, J. A. McGovern and K. G. Richardson, Phys. Lett. B 464, 169 (1999).

[241] D. R. Phillips, S. R. Beane and M. C. Birse, J. Phys. A 32, 3397 (1999).

[242] J. Gegelia, J. Phys. G 25, 1681 (1999).

[243] J. F. Yang and J. H. Huang, Phys. Rev. C 71, 034001 (2005) [Erratum-ibid. C 71, 069901 (2005)].

[244] K. Harada and H. Kubo, Nucl. Phys. B 758, 304 (2006).

[245] K. Harada, H. Kubo and A. Ninomiya, arXiv:nuclth/0702074.

[246] G.P. Lepage, arXiv:nucl-th/9706029.

[247] G.P. Lepage, How to renormalize the Schrödinger equation, talk given at the INT program Effective Field Theories and Effective Interactions, INT, Seattle, USA, June 25-August 2, 2000.

[248] T. D. Cohen and J. M. Hansen, Phys. Lett. B 440, 233 (1998).

[249] T. Frederico, V. S. Timoteo and L. Tomio, Nucl. Phys. A 653, 209 (1999).

[250] D. R. Phillips, I. R. Afnan and A. G. Henry-Edwards,
Phys. Rev. C 61, 044002 (2000)

[251] J. Gegelia and G. Japaridze, Phys. Lett. B 517, 476 (2001).

[252] M. Pavon Valderrama and E. Ruiz Arriola, Phys. Lett. B 580, 149 (2004).

[253] M. Pavon Valderrama and E. Ruiz Arriola, Phys. Rev. C 70, 044006 (2004).

[254] J. Gegelia and S. Scherer, Int. J. Mod. Phys. A 21, 1079 (2006).

[255] M. Pavon Valderrama and E. Ruiz Arriola, Phys. Rev. C 72, 054002 (2005).

[256] M. Pavon Valderrama and E. R. Arriola, Phys. Rev. C 74, 054001 (2006).

[257] M. Pavon Valderrama and E. Ruiz Arriola, Phys. Rev. C 74, 064004 (2006) [Erratum-ibid. C 75, 059905 (2007)].

[258] E. Epelbaum and U.-G. Meißner, arXiv:nuclth/0609037.

[259] D. Djukanovic, J. Gegelia, S. Scherer and M. R. Schindler, Few Body Syst. 41, 141 (2007).

[260] R. Higa, M. Pavon Valderrama and E. Ruiz Arriola, Phys. Rev. C 77, 034003 (2008).

[261] C. J. Yang, C. Elster and D. R. Phillips, Phys. Rev. C 77, 014002 (2008).

[262] B. Long and U. van Kolck, Annals Phys. 323, 1304 (2008).

[263] D. R. Entem, E. Ruiz Arriola, M. Pavon Valderrama and R. Machleidt, Phys. Rev. C 77, 044006 (2008).

[264] M. P. Valderrama and E. R. Arriola, arXiv:0809.3186 [nucl-th].

[265] D. Shukla, D. R. Phillips and E. Mortenson, J. Phys. G 35, 115009 (2008).

[266] S. R. Beane, P. F. Bedaque, M. J. Savage, and U. van Kolck, Nucl. Phys. A 700, 377 (2002).

[267] S. R. Beane, P. F. Bedaque, L. Childress, A. Kryjevski, J. McGuire and U. v. Kolck, Phys. Rev. A 64, 042103 (2001).

[268] M. Bawin and S. A. Coon, Phys. Rev. A 67, 042712 (2003).

[269] E. Braaten and D. Phillips, Phys. Rev. A 70, 052111 (2004).

[270] H.-W. Hammer and B. G. Swingle, Annals Phys. 321, 306 (2006).

[271] D. R. Entem and R. Machleidt, Phys. Rev. C 68, 041001 (2003).

[272] E. Epelbaum, W. Glöckle and U.-G. Meißner, Nucl. Phys. A 747, 362 (2005).

[273] V.G.J. Stoks et al., Phys. Rev. C 48, 792 (1993).

[274] NN-Online program, M. C. M. Rentmeester et al., http://nn-online.org.

[275] SAID on-line program, R. A.Arndt et al., http://gwdac.phys.gwu.edu.

[276] H. Witała, J. Golak, W. Glöckle and H. Kamada, Phys. Rev. C 71, 054001 (2005).

[277] T. Lin, C. Elster, W. N. Polyzou and W. Glöckle, Phys. Lett. B 660, 345 (2008).

[278] T. Lin, C. Elster, W. N. Polyzou, H. Witała and W. Glöckle, Phys. Rev. C 78, 024002 (2008).

[279] H. Kamada and W. Glöckle, Phys. Rev. Lett. 80, 2547 (1998).

[280] E. Epelbaum, U.-G. Meißner, W. Glöckle, and C. Elster, Phys. Rev. C 65, 044001 (2002).

[281] A. Nogga, P. Navratil, B. R. Barrett and J. P. Vary, Phys. Rev. C 73, 064002 (2006).

[282] P. Navratil, V. G. Gueorguiev, J. P. Vary, W. E. Or- 
mand and A. Nogga, Phys. Rev. Lett. 99, 042501 (2007).

[283] C. Hanhart, U. van Kolck and G. A. Miller, Phys. Rev. Lett. 85, 2905 (2000).

[284] S. X. Nakamura, Phys. Rev. C 77, 054001 (2008).

[285] E. Epelbaum, A. Nogga, W. Glöckle, H. Kamada, U.G. Meißner and H. Witała, Eur. Phys. J. A 15, 543 (2002).

[286] K. Ermisch et al., Phys. Rev. C 68, 051001 (2003).

[287] C. Duweke et al., Phys. Rev. C 71, 054003 (2005).

[288] K. Ermisch et al., Phys. Rev. C 71, 064004 (2005).

[289] S. Kistryn et al., Phys. Rev. C 72, 044006 (2005).

[290] H. Witała et al., Phys. Rev. C 73, 044004 (2006).

[291] A. Biegun et al., Acta Phys. Polon. B 37, 213 (2006).

[292] J. Ley et al., Phys. Rev. C 73, 064001 (2006).

[293] E. Stephan et al., Phys. Rev. C 76, 057001 (2007).

[294] D. Huber, H. Witała, A. Nogga, W. Glöckle and H. Kamada, Few Body Syst. 22, 107 (1997).

[295] H. Kamada et al., AIP Conf. Proc. 1011, 59 (2008).

[296] A. Deltuva, A. C. Fonseca and P. U. Sauer, Phys. Rev. C 72, 054004 (2005) [Erratum-ibid. C 72, 059903 (2005)].

[297] A. Deltuva, A. C. Fonseca and P. U. Sauer, Phys. Rev. Lett. 95, 092301 (2005).

[298] C. R. Howell et al., Few-Body Systems 2, 19 (1987).

[299] K. Sagara, H. Oguri, S. Shimizu, K. Maeda, H. Nakamura, T. Nakashima and S. Morinobu, Phys. Rev. C 50, 576 (1994).

[300] G. Rauprich et al., Few-Body Systems 5, 67 (1988).

[301] F. Sperisen et al., Nucl. Phys. A422, 81 (1984).

[302] H. Witała et al., Few-Body Systems 15, 67 (1993).

[303] A. Glombik et al., AIP Conference Proc. 334, 486 (1995).

[304] W. Kretschmer et al., AIP Conference Proc. 339, 335 (1995).

[305] J. Strate et al., Nucl. Phys. A 501, 51 (1989).

[306] H.R. Setze et al., Phys. Lett. B 388, 229 (1996).

[307] G. Rauprich et al., Nucl. Phys. A 535, 313 (1991).

[308] S. A. Coon and H. K. Han, Few Body Syst. 30, 131 (2001).

[309] B. M. Fisher et al., Phys. Rev. C 74, 034001 (2006).

[310] A. Deltuva and A. C. Fonseca, Phys. Rev. Lett. 98, 162502 (2007).

[311] A. Deltuva and A. C. Fonseca, Phys. Rev. C 76, 021001 (2007).

[312] R. Lazauskas, J. Carbonell, A. C. Fonseca, M. Viviani, A. Kievsky and S. Rosati, Phys. Rev. C 71, 034004 (2005).

[313] S. Quaglioni and P. Navratil, arXiv:0804.1560 [nucl-th].

[314] P. Navratil, V. G. Gueorguiev, J. P. Vary, W. E. Ormand, A. Nogga and S. Quaglioni, arXiv:0712.1207 [nucl-th].

[315] T. R. Hemmert, B. R. Holstein and J. Kambor, J. Phys. G 24, 1831 (1998)

[316] E. E. Jenkins and A. V. Manohar, Phys. Lett. B 281, 336 (1992)

[317] V. Pascalutsa, Phys. Rev. D 58, 096002 (1998).

[318] C. Hacker, N. Wies, J. Gegelia and S. Scherer, Phys. Rev. C 72, 055203 (2005).

[319] N. Fettes and U.-G. Meißner, Nucl. Phys. A 679, 629 (2001).

[320] E. Epelbaum, H. Krebs and U.-G. Meißner, Nucl. Phys. A 806, 65 (2008).

[321] H. Krebs, E. Epelbaum and U.-G. Meißner, Eur. Phys. J. A 32, 127 (2007).
[322] N. Kaiser, S. Gerstendorfer and W. Weise, Nucl. Phys. A 637, 395 (1998).

[323] V. R. Pandharipande, D. R. Phillips and U. van Kolck, Phys. Rev. C 71, 064002 (2005).

[324] Particle Data Group, see the website http://pdg.lbl.gov/.

[325] R. A. Arndt, W. J. Briscoe, I. I. Strakovsky and R. L. Workman, Phys. Rev. C 74, 045205 (2006).

[326] H. R. Rubinstein, F. Scheck, and R. H. Sokolov, Phys. Rev. 154, 1608 (1967)

[327] B. C. Tiburzi and A. Walker-Loud, Nucl. Phys. A 764, 274 (2006).

[328] E. Epelbaum, H. Krebs and U.-G. Meißner, Phys. Rev. C 77, 034004 (2008).

[329] S. R. Beane, V. Bernard, E. Epelbaum, U.-G. Meißner and D. R. Phillips, Nucl. Phys. A 720, 399 (2003).

[330] S. R. Beane, V. Bernard, T. S. H. Lee, U.-G. Meißner and U. van Kolck, Nucl. Phys. A 618, 381 (1997).

[331] H. Krebs, V. Bernard and U.-G. Meißner, Eur. Phys. J. A 22, 503 (2004).

[332] V. Baru, J. Haidenbauer, C. Hanhart and J. A. Niskanen, Eur. Phys. J. A 16, 437 (2003).

[333] A. Gardestig and D. R. Phillips, Phys. Rev. C 73, 014002 (2006).

[334] V. Lensky, V. Baru, E. Epelbaum, C. Hanhart, J. Haidenbauer, A. E. Kudryavtsev and U.-G. Meißner, Eur. Phys. J. A 33, 339 (2007).

[335] V. Baru, J. Haidenbauer, C. Hanhart, A. E. Kudryavtsev, V. Lensky and U.-G. Meißner, [arXiv:0711.2748 [nucl-th]].

[336] S. Weinberg, Phys. Lett. B 295, 114 (1992).

[337] T. D. Cohen, J. L. Friar, G. A. Miller and U. van Kolck, Phys. Rev. C 53, 2661 (1996).

[338] V. Bernard, N. Kaiser and U.-G. Meißner, Eur. Phys. J. A 4, 259 (1999).

[339] C. da Rocha, G. Miller and U. van Kolck, Phys. Rev. C 61, 034613 (2000).

[340] C. Hanhart and N. Kaiser, Phys. Rev. C 66, 054005 (2002).

[341] V. Lensky, V. Baru, J. Haidenbauer, C. Hanhart, A. E. Kudryavtsev and U.-G. Meißner, Eur. Phys. J. A 27, 37 (2006).

[342] D. A. Hutcheon et al., Nucl. Phys. A 535, 618 (1991).

[343] P. Heimberg et al., Phys. Rev. Lett. 77, 1012 (1996).

[344] M. Drochner et al. [GEM Collaboration], Nucl. Phys. A 643, 55 (1998).

345] C. Hanhart, Phys. Rept. 397, 155 (2004).

[346] A. K. Opper et al., Phys. Rev. Lett. 91, 212302 (2003).

[347] E. J. Stephenson et al., Phys. Rev. Lett. 91, 142302 (2003).

[348] U. van Kolck, J. A. Niskanen and G. A. Miller, Phys. Lett. B 493, 65 (2000).

[349] A. Gardestig et al., Phys. Rev. C 69, 044606 (2004).

[350] A. Nogga et al., Phys. Lett. B 639, 465 (2006).

[351] H. H. Adam et al. [WASA-at-COSY Collaboration], arXiv:nucl-ex/0411038.

[352] G. A. Miller, A. K. Opper and E. J. Stephenson, Ann. Rev. Nucl. Part. Sci. 56, 253 (2006).

[353] V. Baru, C. Hanhart, A. E. Kudryavtsev and U.G. Meißner, Phys. Lett. B 589, 118 (2004).

[354] V. Lensky, V. Baru, J. Haidenbauer, C. Hanhart, A. E. Kudryavtsev and U.-G. Meißner, Eur. Phys. J. A 26, 107 (2005).

[355] V. Lensky, V. Baru, J. Haidenbauer, C. Hanhart, 
A. E. Kudryavtsev and U.-G. Meißner, Phys. Lett. B 648, 46 (2007).

[356] C. Hanhart, arXiv:nucl-th/0703028.

[357] W. M. Alberico and G. Garbarino, Phys. Rept. 369, 1 (2002).

[358] Bydzovsky, P.; Gal, A.; Mares, J. (Eds.) Lecture Notes in Physics 7242007 (Springer, Heidelberg).

[359] G. Alexander, U. Karshon, A. Shapira, G. Yekutieli, R. Engelmann, H. Filthuth and W. Lughofer, Phys. Rev. 173, 1452 (1968).

[360] T. A. Rijken, V. G. J. Stoks and Y. Yamamoto, Phys. Rev. C 59, 21 (1999).

[361] J. Haidenbauer and U.-G. Meißner, Phys. Rev. C 72, 044005 (2005).

[362] A. Gasparyan, J. Haidenbauer, C. Hanhart and J. Speth, Phys. Rev. C 69 (2004) 034006.

[363] M. J. Savage and M. B. Wise, Phys. Rev. D 53, 349 (1996).

[364] H.-W. Hammer, Nucl. Phys. A 705, 173 (2002).

[365] C. L. Korpa, A. E. L. Dieperink and R. G. E. Timmermans, Phys. Rev. C 65, 015208 (2002).

[366] H. Polinder, J. Haidenbauer and U.-G. Meißner, Nucl. Phys. A 779, 244 (2006).

[367] J. J. de Swart, Rev. Mod. Phys. 35, 916 (1963).

[368] B. Sechi-Zorn, B. Kehoe, J. Twitty and R. A. Burnstein, Phys. Rev. 175, 1735 (1968).

[369] F. Eisele, H. Filthuth, W. Foehlisch, V. Hepp and G. Zech, Phys. Lett. B 37, 204 (1971).

[370] R. Engelmann, H. Filthuth, V. Hepp and E. Kluge, Phys. Lett. 21, 587 (1966).

[371] J. J. de Swart and C. Dullemond, Ann. Phys. 19, 485 (1962).

[372] J. Haidenbauer, U.-G. Meißner, A. Nogga and H. Polinder, Lect. Notes Phys. 724, 113 (2007).

[373] T. Tamagawa et al., Nucl. Phys. A 691, 234 (2001).

[374] J. K. Ahn et al., Phys. Lett. B 633, 214 (2006).

[375] H. Polinder, J. Haidenbauer and U.-G. Meißner, Phys. Lett. B 653, 29 (2007).

[376] H. Takahashi et al., Phys. Rev. Lett. 87 (2001) 212502.

[377] T. A. Rijken and Y. Yamamoto, arXiv:nucl-th/0608074.

[378] Y. Fujiwara, Y. Suzuki and C. Nakamoto, Prog. Part. Nucl. Phys. 58 (2007) 439.

[379] D. Lee, B. Borasoy and T. Schäfer, Phys. Rev. C 70, 014007 (2004).

[380] B. Borasoy, H. Krebs, D. Lee and U.-G. Meißner, Nucl. Phys. A 768, 179 (2006).

[381] B. Borasoy, E. Epelbaum, H. Krebs, D. Lee and U.G. Meißner, Eur. Phys. J. A 31, 105 (2007).

[382] J. W. N. Chen and D. B. Kaplan, Phys. Rev. Lett. 92, 257002 (2004).

[383] D. Lee, Phys. Rev. C 70, 064002 (2004).

[384] E. Wigner, Phys. Rev. 51, 947 (1937).

[385] T. Mehen, I. W. Stewart and M. B. Wise, Phys. Rev. Lett. 83, 931 (1999).

[386] J. W. Chen, D. Lee and T. Schafer, Phys. Rev. Lett. 93, 242302 (2004).

[387] S. Duane, A. D. Kennedy, B. J. Pendleton and D. Roweth, Phys. Lett. B 195, 216 (1987).

[388] D. Lee, Phys. Rev. A 73, 063204 (2006).

[389] B. Borasoy, E. Epelbaum, H. Krebs, D. Lee and U.G. Meißner, Eur. Phys. J. A 34, 185 (2007).

[390] W. T. H. van Oers, J. D. Seagrave, Phys. Lett. B 24, 562 (1967).

[391] S. R. Beane and M. J. Savage, Nucl. Phys. A 717, 91
(2003); Nucl. Phys. A 713, 148 (2003).

[392] N. Fettes, Pion-nucleon physics in chiral perturbation theory, Ph.D. Thesis, Universität Bonn, Germany, 2000, JUL-3814.

[393] E. Epelbaum, U.-G. Meißner and W. Glöckle, arXiv:nucl-th/0208040.

[394] E. Epelbaum, H.-W. Hammer, U.-G. Meißner and A. Nogga, Eur. Phys. J. C 48, 169 (2006).

[395] H.-W. Hammer, D. R. Phillips and L. Platter, Eur. Phys. J. A 32, 335 (2007).

[396] K. G. Wilson, Nucl. Phys. Proc. Suppl. 140, 3 (2005).

[397] V. V. Flambaum and R. B. Wiringa, Phys. Rev. C 76, 054002 (2007).

[398] R. J. Furnstahl, G. Rupak and T. Schafer, Ann. Rev. Nucl. Part. Sci. 58, 1 (2008).

[399] A. E. Nelson and D. B. Kaplan, Phys. Lett. B 192 (1987) 193.

[400] V. Thorsson and A. Wirzba, Nucl. Phys. A 589, 633 (1995).

[401] M. Kirchbach and A. Wirzba, Nucl. Phys. A 604, 395 (1996).

[402] M. Kirchbach and A. Wirzba, Nucl. Phys. A 616, 648 (1997).

[403] J. A. Oller, Phys. Rev. C 65, 025204 (2002).

[404] U.-G. Meißner, J. A. Oller and A. Wirzba, Annals Phys. 297, 27 (2002).

[405] H. Leutwyler, Phys. Rev. D 49, 3033 (1994).

[406] E. G. Drukarev and E. M. Levin, Nucl. Phys. A 511, 679 (1990) [Erratum-ibid. A 516, 715 (1990)].

[407] R. J. Furnstahl, D. K. Griegel and T. D. Cohen, Phys. Rev. C 46, 1507 (1992).

[408] M. C. Birse, J. Phys. G 20, 1537 (1994).

[409] R. D. Pisarski and M. Tytgat, Phys. Rev. D 54, 2989 (1996).

[410] H. Gilg et al., Phys. Rev. C 62, 025201 (2000).

[411] K. Itahashi et al., Phys. Rev. C 62, 025202 (2000).

[412] J.A. Oller, private communication

[413] A. Lacour, U.-G. Meißner, J.A. Oller, A. Wirzba, in preparation.

[414] L. Girlanda, A. Rusetsky and W. Weise, Annals Phys. 312, 92 (2004).

[415] L. Girlanda, A. Rusetsky and W. Weise, Nucl. Phys. A 755, 653 (2005).

[416] N. Kaiser, S. Fritsch and W. Weise, Nucl. Phys. A 697, 255 (2002).

[417] S. Fritsch, N. Kaiser and W. Weise, Nucl. Phys. A 750, 259 (2005).

[418] N. Kaiser, S. Fritsch and W. Weise, Nucl. Phys. A 700, 343 (2002).

[419] M. Lutz, B. Friman and C. Appel, Phys. Lett. B 474, 7 (2000).

[420] N. Kaiser, Nucl. Phys. A 709, 251 (2002).

[421] N. Kaiser, Phys. Rev. C 68, 054001 (2003).

[422] N. Kaiser and W. Weise, Phys. Rev. C 71, 015203 (2005).

[423] N. Kaiser and W. Weise, Nucl. Phys. A 804, 60 (2008).

[424] N. Kaiser, P. de Homont and W. Weise, Phys. Rev. C 77, 025204 (2008).

[425] N. Kaiser and W. Weise, arXiv:0808.0856 [nucl-th].

[426] N. Kaiser, S. Fritsch and W. Weise, Nucl. Phys. A 724, 47 (2003).

[427] P. Finelli, N. Kaiser, D. Vretenar and W. Weise, Eur. Phys. J. A 17, 573 (2003).

[428] P. Finelli, N. Kaiser, D. Vretenar and W. Weise, Nucl. 
Phys. A 735, 449 (2004).

[429] P. Finelli, N. Kaiser, D. Vretenar and W. Weise, Phys. Lett. B 658, 90 (2007).

[430] M.V. Zhukov, B.V. Danilin, D.V. Fedorov, J.M. Bang, I.J. Thompson, and J.S. Vaagen, Phys. Rep. 231, 151 (1993).

[431] A.S. Jensen, K. Riisager, D.V. Fedorov, and E. Garrido, Rev. Mod. Phys. 76, 215 (2004).

[432] C. A. Bertulani, H.-W. Hammer and U. Van Kolck, Nucl. Phys. A 712, 37 (2002).

[433] P. F. Bedaque, H.-W. Hammer and U. van Kolck, Phys. Lett. B 569, 159 (2003).

[434] R. Higa, arXiv:0809.5157 [nucl-th].

[435] R. Higa, H.-W. Hammer and U. van Kolck, Nucl. Phys. A 809, 171 (2008).

[436] H.-W. Hammer and R. Higa, Eur. Phys. J. A 37, 193 (2008).

[437] D. V. Federov, A. S. Jensen and K. Riisager, Phys. Rev. Lett. 73, 2817 (1994).

[438] A. E. A. Amorim, T. Frederico and L. Tomio, Phys. Rev. C 56, R2378 (1997).

[439] I. Mazumdar, V. Arora and V. S. Bhasin, Phys. Rev. C 61, 051303 (2000).

[440] D. L. Canham and H. W. Hammer, Eur. Phys. J. A 37, 367 (2008).

[441] F. M. Marques et al., Phys. Rev. C 64, 061301 (2001).

[442] M. T. Yamashita, T. Frederico and L. Tomio, Phys. Lett. B 660, 339 (2008).

[443] I. Mazumdar, A. R. P. Rau and V. S. Bhasin, Phys. Rev. Lett. 97, 062503 (2006).

[444] A. Cobis, A.S. Jensen and D.V. Fedorov, J. Phys. G 23, 401 (1997).

[445] D.V. Fedorov and A.S. Jensen, Nucl. Phys. A 697, 783 (2002).

[446] J.G. Congleton, J. Phys. G 18, 339 (1992).

[447] C.A. Bertulani and G. Baur, Phys. Rep. 163, 299 (1988).

[448] S. K. Bogner, T. T. S. Kuo and A. Schwenk, Phys. Rept. 386, 1 (2003).

[449] E. Epelbaum, W. Glöckle and U.-G. Meißner, Phys. Lett. B 439, 1 (1998).

[450] E. Epelbaum, W. Glöckle, A. Krüger and U.G. Meißner, Nucl. Phys. A 645, 413 (1999).

[451] S. Okubo, Prog. Theor. Phys. 12, 603 (1954).

[452] N. Fukuda, K. Sawada, and M. Taketani, Prog. Theor.
Phys. 12, 156 (1954).

[453] S. K. Bogner, R. J. Furnstahl and R. J. Perry, Phys. Rev. C 75, 061001 (2007).

[454] E. D. Jurgenson and R. J. Furnstahl, arXiv:0809.4199 [nucl-th].

[455] B. J. Schaefer, M. Wagner, J. Wambach, T. T. S. Kuo and G. E. Brown, Phys. Rev. C 73, 011001 (2006).

[456] H. Dapo, B. J. Schaefer and J. Wambach, Eur. Phys. J. A 36, 101 (2008).

[457] S. Fujii, E. Epelbaum, H. Kamada, R. Okamoto, K. Suzuki and W. Glöckle, Phys. Rev. C 70, 024003 (2004).

[458] A. Schwenk and J. D. Holt, AIP Conf. Proc. 1011, 159 (2008).

[459] S. K. Bogner, A. Schwenk, R. J. Furnstahl and A. Nogga, Nucl. Phys. A 763, 59 (2005).

[460] S. K. Bogner, R. J. Furnstahl, S. Ramanan and A. Schwenk, Nucl. Phys. A 773, 203 (2006).

[461] R. J. Furnstahl, arXiv:nucl-th/0702040.

[462] G.F. Bertsch, D.J. Dean, and W. Nazarewicz, SciDAC Review 6, 42 (2007).

[463] R. Brockmann and J. Frank, Phys. Rev. Lett. 68, 1830 (1992).

[464] B. D. Serot and J. D. Walecka, Adv. Nucl. Phys. 16, 1 (1986).

[465] H. M. Muller, S. E. Koonin, R. Seki and U. van Kolck, Phys. Rev. C 61, 044320 (2000).

[466] B. Borasoy, E. Epelbaum, H. Krebs, D. Lee and U.G. Meißner, Eur. Phys. J. A 35, 357 (2008).

[467] S. Giorgini, L.P. Pitaevskii, and S. Stringari, Rev. Mod. Phys. 80, 1215 (2008).

[468] E. Epelbaum, H. Krebs, D. Lee and U.-G. Meißner, forthcoming.

[469] B. Friedman and V. R. Pandharipande, Nucl. Phys. A 361 (1981) 502.

[470] A. Akmal, V. R. Pandharipande and D. G. Ravenhall, Phys. Rev. C 58, 1804 (1998).

[471] J. Carlson, J. . J. Morales, V. R. Pandharipande and D. G. Ravenhall, Phys. Rev. C 68, 025802 (2003).

[472] A. Schwenk and C. J. Pethick, Phys. Rev. Lett. 95, 160401 (2005).

[473] A. Gezerlis and J. Carlson, Phys. Rev. C 77, 032801 (2008).

[474] D. Lee, Phys. Rev. C 78, 024001 (2008). 